University of South Florida

DIGITAL COMMONS

Digital Commons @ University of

@ UNIVERSITY OF SOUTH FLORIDA

South Florida

USF Tampa Graduate Theses and Dissertations

USF Graduate Theses and Dissertations

10-13-2005

\title{
Nanomaterial Sensing Layer Based Surface Acoustic Wave Hydrogen Sensors
}

Krishnan Srinivasan

University of South Florida

Follow this and additional works at: https://digitalcommons.usf.edu/etd

Part of the American Studies Commons

\section{Scholar Commons Citation}

Srinivasan, Krishnan, "Nanomaterial Sensing Layer Based Surface Acoustic Wave Hydrogen Sensors" (2005). USF Tampa Graduate Theses and Dissertations.

https://digitalcommons.usf.edu/etd/873

This Thesis is brought to you for free and open access by the USF Graduate Theses and Dissertations at Digital Commons @ University of South Florida. It has been accepted for inclusion in USF Tampa Graduate Theses and Dissertations by an authorized administrator of Digital Commons @ University of South Florida. For more information, please contact digitalcommons@usf.edu. 
Nanomaterial Sensing Layer Based Surface Acoustic Wave Hydrogen Sensors

by

\author{
Krishnan Srinivasan
}

A thesis submitted in partial fulfillment of the requirements for the degree of Master of Science in Electrical Engineering Department of Electrical Engineering College of Engineering University of South Florida

Co-Major Professor: Venkat R. Bhethanabotla, Ph.D. Co-Major Professor: Paris H. Wiley, Ph.D. Thomas Weller, Ph.D. Babu Joseph, Ph.D.

Date of Approval:

October 13, 2005

Keywords: Saw Resoantor, Tobacco Mosaic Virus, Carbon Nanotubes, Palladium, Adsorption

(C) Copyright 2005, Krishnan Srinivasan 


\section{DEDICATION}

This dissertation is dedicated to my parents, G. Srinivasan and Lakshmi Srinivasan and to my sister Kavita Srinivasan. Their love, support and patience have always been the foundations of my inspiration. 


\section{ACKNOWLEDGEMENTS}

I wish to express sincere appreciation to my major professor Dr. Venkat Bhethanabotla for his valuable guidance and support during this thesis work. Thanks to Dr Paris $\mathrm{H}$. Wiley, Dr. Thomas Weller and Dr. Babu Joseph for agreeing to serve on my supervisory committee.

I would like to thank Stefan Cular and Randy Williams for helping me at every step. I also appreciate Dr. Michael T Harris and Sang-Yup Lee of Purdue University for providing the Pd-TMV materials.

Special thanks are extended to Sensors Research Group and my friends for their support and encouragement throughout this research. 


\section{TABLE OF CONTENTS}

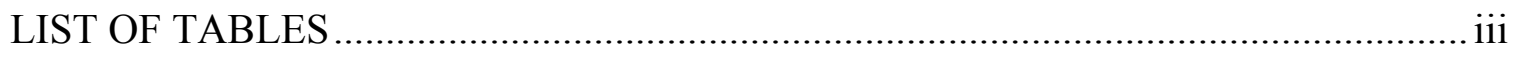

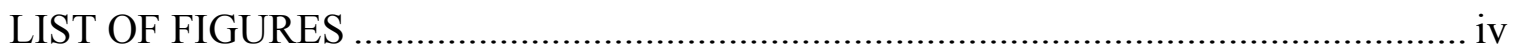

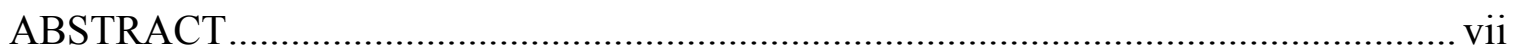

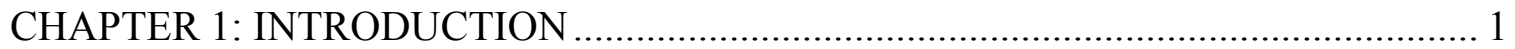

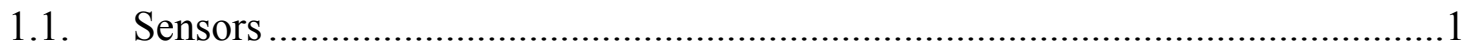

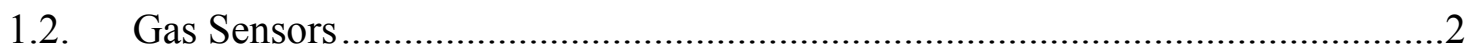

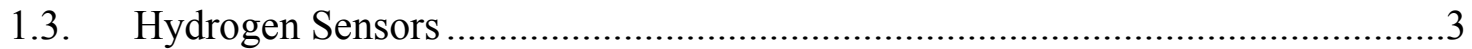

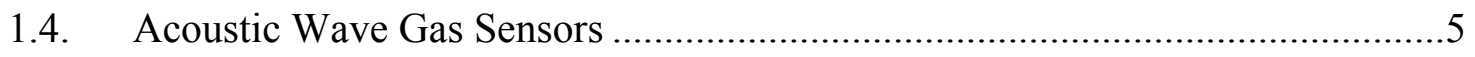

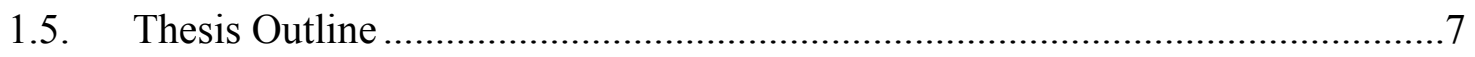

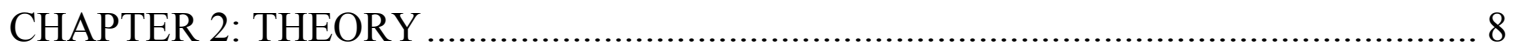

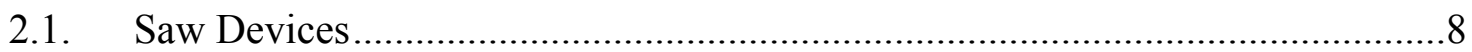

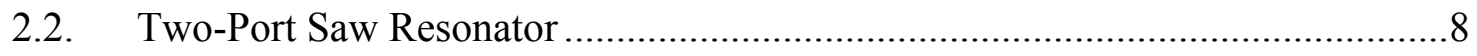

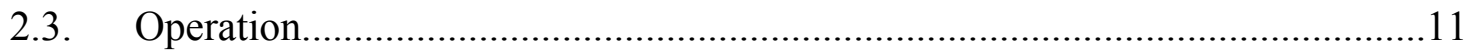

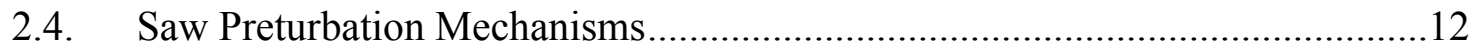

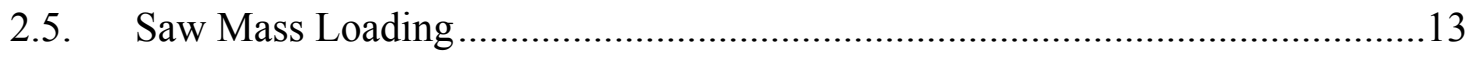

2.6. Saw Acousto-electric Effect ……………..............................................15

2.7. Saw Sensor Operation...............................................................................

2.8. Oscillator Based Measurement Method ......................................................18

2.9. Type of Measurement ……….............................................................19

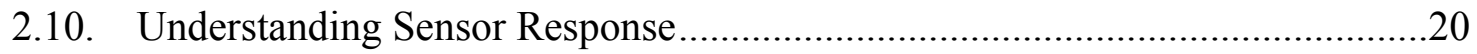

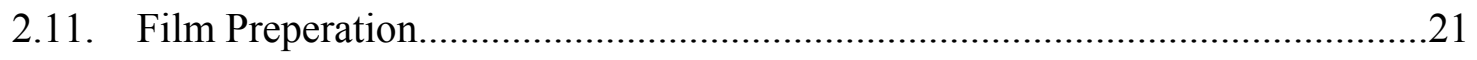

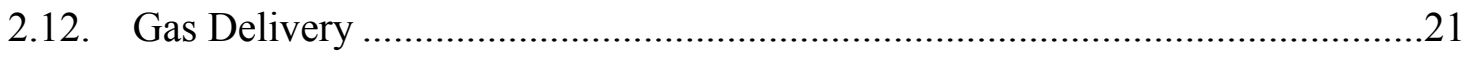

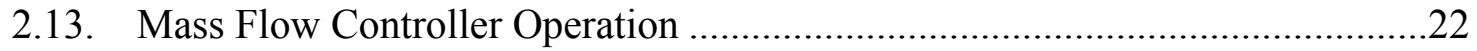

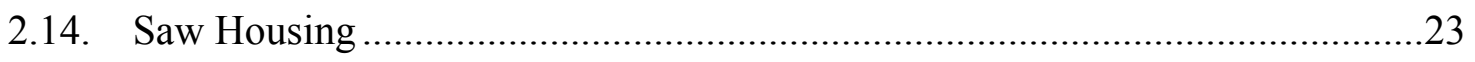




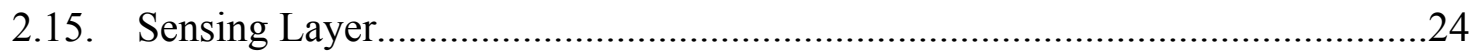

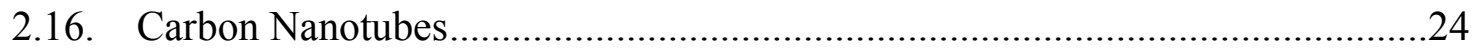

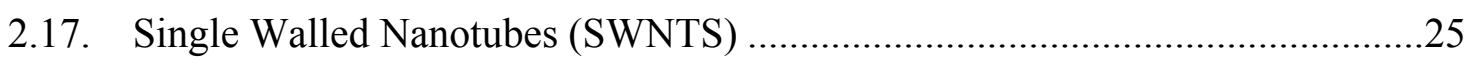

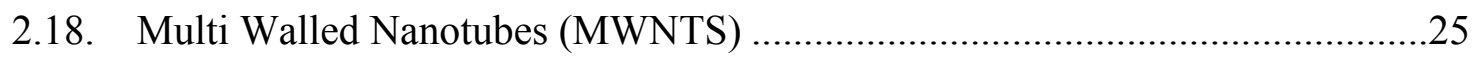

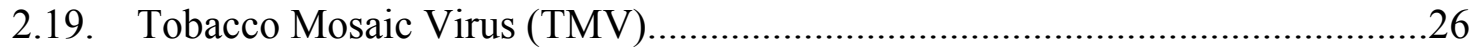

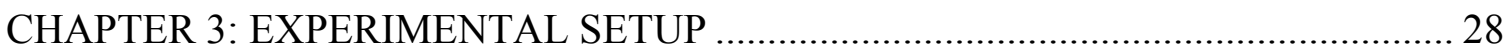

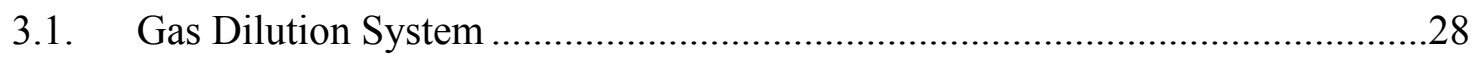

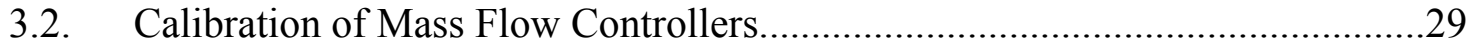

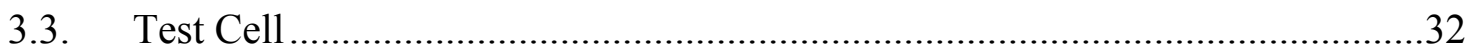

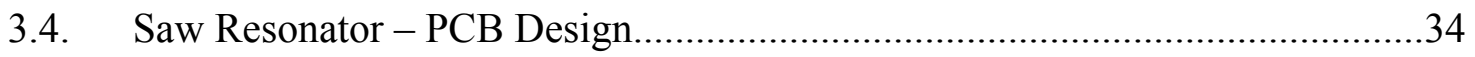

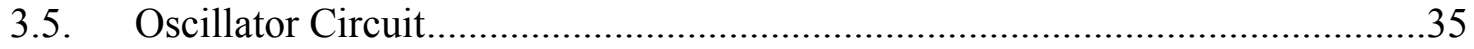

3.5.1 Amplifier................................................. 36

3.5.2 Filter.......................................................... 36

3.5.3 Attenuator.............................................. 36

3.5.4 Phase Shifter............................................ 36

3.5.5 Coupler.................................................... 38

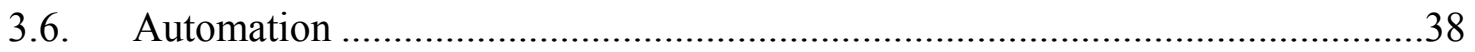

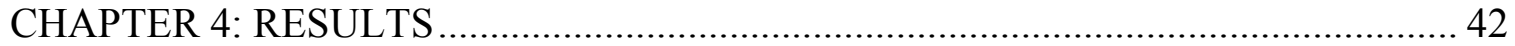

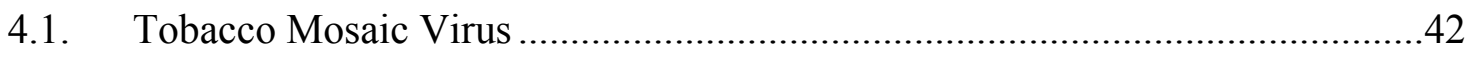

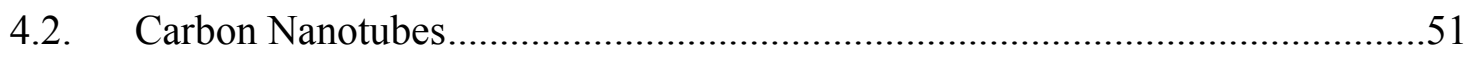

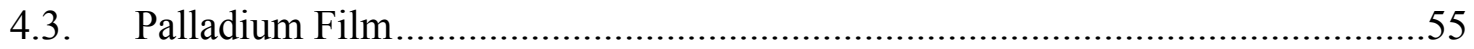

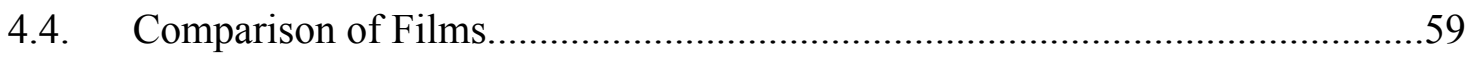

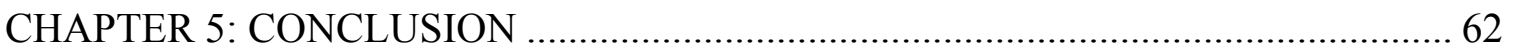

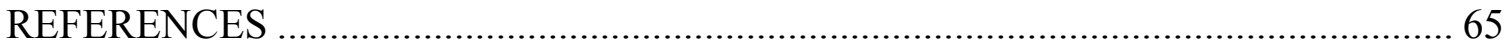

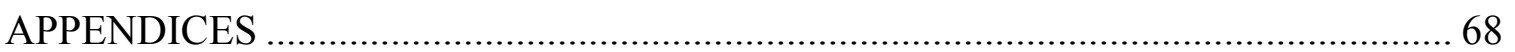

APPENDIX A - PRINTED CIRCUIT FABRICATION ................................................ 69 


\section{LIST OF TABLES}

Table 1.1. Measurement Format ............................................................................

Table 2.1. Measurement Techniques Used by Different Gas Delivery Systems..............22

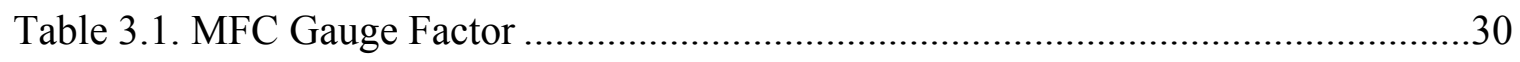

Table 3.2. Scaling Factor for Hydrogen Based on Flow Rates........................................32

Table 4.1. SAW Resonator Frequency and Attenuation Measurements after Pd-TMV

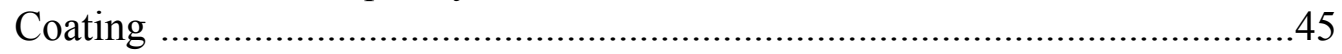

Table 4.2. SAW Resonator Frequency and Attenuation Measurements after Pd-SWNT Coating

Table 4.3. SAW Resonator Frequency and Attenuation Measurements after Pd Coating .55

Table 4.4. Comparison of Different Sensor Parameters for the Three Sensing Films.......60 


\section{LIST OF FIGURES}

Figure 2.1. (A) Delay- Line (B) Two-Port SAW Resonator (C) Single-Port SAW Resonator....

Figure 2.2. (A) Lumped Equivalent Circuit for $0^{\circ}$ Phase Shift between Input/Output IDT's at Resonance. (B) Ideal 1:-1 Transformer Included for $180^{\circ}$ Phase Shift a Resonance.

Figure 2.3. Two-Port SAW Resonator................................................................... 10

Figure 2.4. Equivalent Circuit Model Describing the Interaction between SAW and Charge Carriers on Film Overlay ................................................... 15

Figure 2.5. Oscillator Block Diagram......................................................................19

Figure 2.6. The Mass Flow Controller.....................................................................23

Figure 2.7. S.E.M. Image of Carbon Nanotubes..........................................................26

Figure 2.8. Microscopic Image of Single Walled Carbon Nanotubes .............................26

Figure 2.9. (A) Armchair (5,5), (B) Zigzag (10,0) (C) Chiral $(10,5)$.............................27

Figure 2.10. High Resolution T.E.M. Image of Multi-Walled Carbon Nanotubes ..........27

Figure 2.11. Electron Microscope View of a Single TMV Tube....................................27

Figure 2.12. T.E.M. View of a Pd Coated TMV Sample (Scale Bar- 200nm) .................27

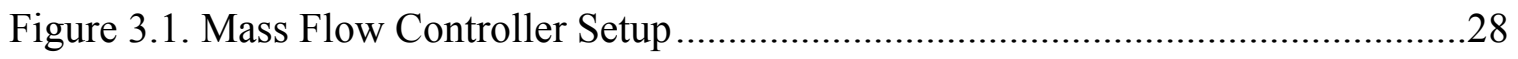

Figure 3.2. The Complete Experimental Setup.........................................................29

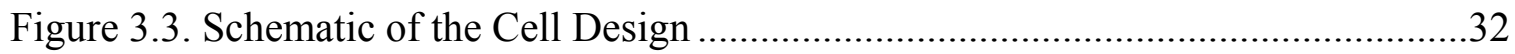

Figure 3.4. The Machined Stainless Steel Cell ..........................................................33

Figure 3.5. The Top and Bottom Surface of PCB Designed in AutoCAD. The Bottom Side is One Facing the Cell............................................................34

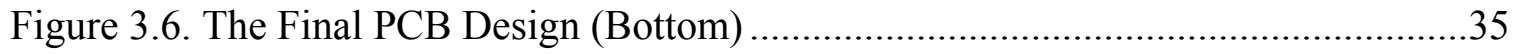

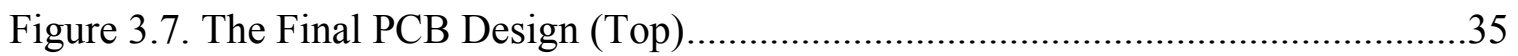

Figure 3.8. PCB Design for Two 180 Degree Phase Shifter Connected in Series ............37 iv 
Figure 3.9. The Final PCB Design of the Phase Shifter

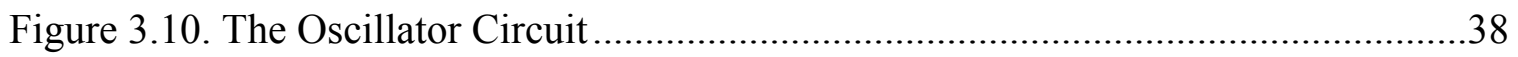

Figure 3.11. The LABVIEW Program for Writing Data Automatically into a Text File .39

Figure 3.12. The Front Panel Display of LABVIEW Program .40

Figure 3.13. The LABVIEW Program to Control the Mass Flow Controllers and Log Sensor Parameters 41

Figure 4.1. The Two-Port Characteristics of SAW RP1239 Two-Port Resonator .42

Figure 4.2. The Two-Port Characteristics of SAW RP1239 Two-Port Resonator after TMV-Pd Coating

Figure 4.3. The Two-Port Characteristics of SAW RP1239 Two-Port Resonator after $\mathrm{Si}_{2}$ Coating .44

Figure 4.4. The Response of Pd Coated TMV to 0.2-2.5 \% Hydrogen ..... .46

Figure 4.5. The Response Times for Pd Coated TMV Resonator in Response to $0.2-2.5 \%$ Hydrogen .46

Figure 4.6. The Frequency Shifts Produced for Two Different Runs at Various Hydrogen Concentrations.

Figure 4.7. The Sensing Film Response to $2-4.5 \%$ Hydrogen .48

Figure 4.8. The Response Times of Sensing Film for 2-4.5\% Hydrogen 48

Figure 4.9. The Frequency Shift Produced in the Sensing Film 2.5-4.5\% Hydrogen.

Figure 4.10. Cracking of Palladium Film Upon Hydrogen Sorption/Desorption..... .50

Figure 4.11. Device Surface Coated with Palladium Nanoparticle-Coated Tobacco Mosaic Virus Film Free of Rearrangement.

Figure 4.12. The Curve Showing the Response of Pd Coated SWNTs Response to Hydrogen

Figure 4.13. Response Times for Pd-SWNTs Coated SAW Resonator to 0.5-2.5\% Hydrogen Exposures

Figure 4.14. Frequency Shifts for Pd-SWNTs Coated SAW Resonator to 0.2-2.5\% Hydrogen Exposures

Figure 4.15. Frequency Shifts for Pd-SWNTs Coated SAW Resonator to $1.5 \%$ Hydrogen 
Figure 4.16. Frequency Shifts for Pd-SWNTs Coated SAW Resonator to $1 \%$

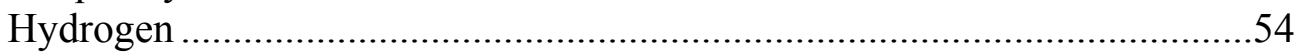

Figure 4.17. The Curve Showing the Response of Pd Film on Exposure to 0.2$2.5 \%$ Hydrogen.

Figure 4.18. Frequency Shifts for Pure Pd Coated SAW Resonator to 1-2.5\% Hydrogen Exposures

Figure 4. 19. Response Times for Pure Pd Coated SAW Resonator to 1-2.5\% Hydrogen Exposures

Figure 4.20. Response Times for three different runs of Pure Pd Coated SAW Resonator to 1-2.5\% Hydrogen Exposures .58

Figure 4.21. Frequency Shifts for three different runs of Pure Pd Coated SAW Resonator to 1-2.5\% Hydrogen Exposures .58

Figure 4.22. The Response Times Observed for the Sensing Films for $63 \%$ of Full Response.

Figure 4.23. The Frequency Shifts Observed for the Sensing Films for $63 \%$ of Full Scale Response .60 


\title{
Nanomaterial Sensing Layer Based Surface Acoustic Wave Hydrogen Sensors
}

\section{Krishnan Srinivasan}

\begin{abstract}
This thesis addresses the design and use of suitable nanomaterials and surface acoustic wave sensors for hydrogen detection and sensing.

Nanotechnology is aimed at design and synthesis of novel nanoscale materials. These materials could find uses in the design of optical, biomedical and electronic devices. One such example of a nanoscale biological system is a virus. Viruses have been given a lot of attention for assembly of nanoelectronic materials. The tobacco mosaic virus (TMV) used in this research represents an inexpensive and renewable biotemplate that can be easily functionalized for the synthesis of nanomaterials. Strains of this virus have been previously coated with metals, silica or semiconductor materials with potential applications in the assembly of nanostructures and nanoelectronic circuits. Carbon nanotubes are another set of well-characterized nanoscale materials which have been widely investigated to put their physical and chemical properties to use in design of transistors, gas sensors, hydrogen storage cells, etc. Palladium is a well-known material for detection of hydrogen. The processes of absorption and desorption are known to be reversible and are known to produce changes in density, elastic properties and conductivity of the film. Despite these advantages, palladium films are known to suffer from problems of peeling and cracking in hydrogen sensor applications. They are also required to be cycled for a few times with hydrogen before they give reproducible responses.
\end{abstract}

The work presented in this thesis, takes concepts from previous hydrogen sensing techniques and applies them to two nanoengineered particles ( $\mathrm{Pd}$ coated TMV and $\mathrm{Pd}$ coated SWNTs) as SAW resonator sensing materials. Possible sensing 
enhancements to be gained by using these nanomaterial sensing layers are investigated. SAW resonators were coated with these two different nano-structured sensing layers ( $\mathrm{Pd}$ TMV and Pd-SWNT) which produced differently useful hydrogen sensor responses. The Pd-TMV coated resonator responded to hydrogen with nearly constant increases in frequency as compared to the Pd-SWNT coated device, which responded with concentration-dependent decreases in frequency of greater magnitude upon hydrogen exposure. The former behavior is more associated with acousto-electric phenomena in SAW devices and the later with mass loading. The $99 \%$ response times were $30-40$ seconds for the Pd-TMV sensing layer and approximately 150 seconds for the Pd-SWNT layer. Both the films showed high robustness and reversibility at room temperature. When the Pd film was exposed to hydrogen it was observed that it produced decreases in frequency to hydrogen challenges, conforming to mass loading effect. It was also observed that the Pd film started degrading with repeated exposure to hydrogen, with shifts after each exposure going smaller and smaller. 


\section{CHAPTER 1: INTRODUCTION}

\subsection{Sensors}

A Sensor can be defined as a biological organ or a technological device capable of detecting and/or sensing a chemical compound or physical conditions. Most sensors convert these physical parameters into a meaningful format, usually an electrical signal which can be easily interpreted. There are two types of sensors, biological sensors and artificial or man made sensors. Human body can be said to be a biological sensor, some of which include eyes, nose, ears, tongue etc. All these sensors respond to some form of external stimuli. For example, when we enter a bright rooms from a dark one, human eyes shrink making it easy to adjust to higher intensity of light, or the nose, which is highly sensitive to different types of smells, thus making it easier for us to distinguish among them. As we head toward the future developing newer products and technologies there is a desire to develop better sensors capable of doing whole range of sensing applications some of which imitate biological sensors. Such sensors are called artificial sensors. These sensors are either direct indicating such as a mercury thermometer or an electrical meter, or they may be coupled with an indicator to make the value sensed to be represented in a more meaningful format. Most artificial sensors are classified on the basis of energy they detect. For example:

1. Light sensors: Capable of converting light energy into electrical energy. Photocell, photodiode, image sensor are some of light sensors. Gas Sensors and liquid flow meter: Capable of detecting gas and liquid flow. Flow sensor, anemometer, gas meter and flow meter are some examples of such sensors.

2. Temperature sensor: Capable of detecting either direct temperature of changes in temperature. Mercury thermometer, thermocouple and thermostats are examples of such sensors. 
3. Motion Sensors: Capable of detecting and measuring linear or angular motion. Tachometer, radar gun, speedometer are examples of such sensors.

4. Pressure sensor: Capable of measuring direct or changes in pressure. Barometer, barograph, pressure gauge are examples of such sensors.

5. Chemical Sensors: Capable of detecting certain chemical reactions and detecting presence of chemicals.

6. Orientation Sensor: Capable of detecting orientation in two or three dimensional space. Gyroscope, artificial horizon, ring laser gyroscope are example of such sensors.

These are some commonly used sensors with hundreds of more different type of sensors existing in industry.

\subsection{Gas Sensors}

Gas sensors are devices that interact with gases and give data in a more meaningful format. Important measurement specifications to consider when looking for gas sensors as detectors include the response time, the distance, and the flow rate. The response time is the amount of time required for process the signal from the instant the gas comes in contact. Distance is the maximum distance between the position of sensors and gas leak to which the device is sensitive, while flow rate in the necessary flow rate of the gas across the sensor to which it is sensitive. Some of the commonly used gas sensors are for ammonia, aerosols, arsine, bromine, carbon dioxide, carbon monoxide, chlorine, chlorine dioxide, diborane, dust, fluorine, germane, halocarbons or refrigerants, hydrocarbons, hydrogen, hydrogen chloride, hydrogen cyanide, hydrogen fluoride, hydrogen selenide, hydrogen sulfide, mercury vapor, nitrogen dioxide, nitrogen oxides, nitric oxide, organic solvents, oxygen, ozone, phosphine, silane, sulfur dioxide and water vapor. The measurements made by such instruments could include percent volume, trace, leakage, consumption, density, spectra, lower explosive limit or flammable limit. 
Table 1.1. Measurement Format

\begin{tabular}{|c|c|}
\hline Gas Property & Format(Units) \\
\hline Leakage & $\mathrm{ml} / \mathrm{min}$ \\
\hline Trace & Concentration- $\mathrm{ppm}$ \\
\hline Consumption & $\mathrm{ml} / \mathrm{L} / \mathrm{hr}$ \\
\hline Gas Spectra & Chromatograph \\
\hline Density & $\mathrm{Mg} / \mathrm{m}^{3}$ \\
\hline
\end{tabular}

Common outputs from gas sensors include analog voltage, pulse signals, analog currents and switch or relays. Some key factors to be considered for gas sensors include temperature and operating humidity.

\section{Market for Gas Sensors}

In the year 2004, the global market for gas sensors and gas metering equipment was estimated to be about at $\$ 2.8$ billion and is expected to grow at average annual growth rate of $5.9 \%$ to reach $\$ 3.8$ billion in 2009 . Of these, the sensor monitoring market is estimated to grow at an average annual growth rate of $6.3 \%$ to reach $\$ 1.8$ billion in 2009 from $\$ 1.3$ billion in 2004. The secondary instrumentation is also expected to reach $\$ 512$ million in 2009 , rising at an average annual growth rate of $7.2 \%$ [1].

\subsection{Hydrogen Sensors}

Fossil fuel has been in use for a long time sustaining economies the world over, but with increasing consumption and price, and ever decreasing reserves, it has led us to explore for newer alternatives of fuel. Hydrogen is an important industrial fuel being used as feedstock for chemical, food, metallurgical, and electronic industries with current production exceeding 500 billion $\mathrm{Nm}^{3}$ [2]. Hydrogen is a clean, reliable, and affordable energy supply that can sustain world economy and environment. There has been ever growing research in utilizing hydrogen energy for transportation, electric power generation, and portable electronic devices such as mobile phones and laptop computers 
[3]. With prototype hydrogen-fuelled cars on the road there is an increasing need for large scale production, distribution and network for hydrogen gas.

An economy powered by hydrogen has many advantages:

1. It is a clean technology. The only byproduct is water thus eliminating environmental dangers such as oil spills.

2. If the hydrogen comes from the electrolysis of water, then hydrogen adds no greenhouse gases to the environment. There is a perfect cycle wherein electrolysis produces hydrogen from water, and the hydrogen recombines with oxygen to create water and power in a fuel cell. However, a non-polluting energy source is necessary for he electrolysis, such as solar photoelectric.

3. Hydrogen can be produced anywhere that you have electricity and water, thus enabling distributed production.

Though with numerous advantages offered by an economy sustained by hydrogen there are also a number of problems that need to be addressed. Cost and performance issues associated with hydrogen energy systems will need to be addressed in tandem with customer awareness and acceptance [3]. Key issues include consumer safety, convenience and affordability. Developing hydrogen as a major energy carrier, however, will require solutions to many challenges in the areas of infrastructure, technology, and economics. Cost, safety, and reliability issues will influence the planning, design, and development of central versus distributed production and delivery. One major problems of hydrogen is if mixed with air in the ratio 4.65:93.9 vol\% it could be explosive $[4,5]$.

Different types of hydrogen sensors [4]:

1. Piezoelectric sensors: Such sensors allow transduction between electrical and acoustic energies based on piezoelectric crystals. Commonly used sensors in this category include quartz crystal microbalance (QCM) and surface acoustic wave sensor (SAW). Whenever there is absorption of hydrogen by palladium film on be 
these devices there is an attenuation of signal and change in frequency which can be calibrated to determine the amount of gas absorption.

2. Fiber optic sensors: In such sensors the palladium coated fiber produces a change in light guided within it upon of hydrogen absorption. The response can be measured in terms of absorbance, reflectance, luminescence, or scattering

3. Electrochemical sensors: Solid state electrochemical cells are used for continuous measurements of flowing hydrogen. Since the hydrogen provides a proton path, equivalent to electrolytic ionic conduction the measured, current can be used to monitor hydrogen/proton concentration.

4. Semiconductor Sensors: The semiconductor chemical sensor is based on the metal oxide semiconductor (MOS) junction principle. MOS sensors are of two types, MOS capacitors and MOS transistors. Hydrogen molecules when adsorbed on a metal surface act as dipoles and they give rise to macroscopic measurable voltage drop. The dipole layer shifts the energy levels at the metal-insulator interface. The voltage is recorded as a function of time for various hydrogen concentrations.

5. Resistive sensors: Such sensors are sensitive to change in resistance of a metal film upon the absorption of hydrogen. Such sensors use very simple films of Pd, tin oxide, etc where change in resistance is related to the hydrogen concentration. The oxide sensors often operate at very high temperatures.

\subsection{Acoustic Wave Gas Sensors}

Acoustic wave sensors have been in research for a long time. These are constructed primarily on two platforms, surface or bulk acoustic wave (SAW or BAW) devices used as delay-lines or resonators and thickness shear mode (TSM) bulk wave resonators. These devices are typically made on a piezoelectric substrate. This substrate is coated with a gas absorbing film. The absorption of target gas molecules by the film cause the signal to be attenuated and the frequency to change. The use of BAW devices was first reported in year 1964 by King [6]. He coated the device with stationary phase materials and incorporated it into gas liquid chromatograph instrument as detector. Similar concept was used for many years in designing $\mathrm{NH}_{3}, \mathrm{SO}_{2}, \mathrm{CO}_{2}$ sensors. The first 
use of SAW device for chemical sensing was published in 1979 by Wohltjen and Dessy [7] [8]. They discussed the deposition of sorptive films on the surface. It was soon established that the sensitivity of SAW devices was higher than BAW device because of their higher frequencies. It was also shown theoretically that the absolute mass sensitivity of 3e-15 g could be achieved using SAW devices [9]. SAW devices are favored for use in chemical sensing applications because of their small size, low cost, high sensitivity and reliability. Most SAW chemical sensors monitor changes of the SAW phase velocity and attenuation as the vapor interacts with the sensing layer. The shift in the phase velocity and/or attenuation is measured by recording the frequency and insertion loss of the SAW device, respectively. Various effects, including mass loading, viscoelastic loading, and acousto-electric coupling contribute to SAW sensor response. Typical chemical sensors take advantage of one or more of these mechanisms in designing gas sensors.

Earlier work by Christofides and Mandelis [4] has reviewed the theoretical and experimental development of solid state hydrogen detectors. They compared some of the existing technologies and examined the role of Pd in hydrogen absorption. Their review indicates that a Pd-fiber optic sensor was most sensitive while a Pd-photo-pyro-electric sensor to be most economical, had high signal to noise ratio at wide temperature range and was second best in sensitivity at room temperature.

More closely related to this research, Jakubik and co-workers $[10,11]$ have contributed to the understanding of the mass and acousto-electric effect response of phthalocyanine layers on SAW delay-line devices. They have studied responses of different bi-layer films of phthalocyanines and Pd to hydrogen to optimize the response. Their work showed that the best stability between the sensing layer and analyte occurred for copper (II) phthalocyanine and Pd bilayers while best responses were seen using the metal-free phthalocyanine, (hydrogen phthalocyanine) and Pd as the bi-layer sensing film. They showed that though the interaction between the sensing film and analyte was stable, sensitive, and reversible, the interaction of the layers was highly dependent on temperature in an inverse relationship. 
Current work of this group involves an explanation of the interaction of bi-layer films and the role of the film thickness with regard to SAW device response.

Penza and co-workers [12, 13, and14] have used SAW resonators, rather than delay-lines, coated with different vapor sensing layers for detection of organic and inorganic compounds. They have shown that resonators coated with funtionalized singlewalled carbon nanotubes (SWNTs) and multi-walled carbon nanotubes (MWNTs) when used to detect volatile organic compounds display sensitivities that are three to four orders of magnitude larger than polymer-coated SAW devices. While other work utilizing both SAW delay-lines and resonators with polymer and other sensing layers exists in the literature for gas and vapor sensors, this work of Penza et al. is perhaps the only example of orders of magnitude enhancement in sensitivity when nanomaterial sensing layers are employed.

Recently, various metal custer-coated biomolecular nanotubes were synthesized using a genetically engineered Tobacco mosaic virus (TMV) biotemplate. TMV is a wellstudied tubular plant virus $300 \mathrm{~nm}$ in length and $18 \mathrm{~nm}$ in diameter. Monodispersed stable Pd cluster-coated nanotubes were produced from the engineered TMV, exploiting its well-defined tubular structure and the metal binding specificity conferred by engineered cysteine derived sulfhdryl groups. This virus-based nanotube is a promising alternative to carbon nanotubes.

\subsection{Thesis Outline}

Chapter 2 presents the background information needed for understanding how SAW sensors work and how to exploit their parameters for designing sensors. Chapter 3 presents the experimental setup used for designing sensors. The experimental results are

presented in Chapter 4, and Chapter 5 contains a discussion of the experimental results, suggestions and future work. 


\section{CHAPTER 2: THEORY}

\subsection{Saw Devices}

There are two categories of SAW devices, resonators and delay-lines. Delay-lines are two- port devices having two separate sets of Interdigital Transducers (IDTs) deposited on a piezoelectric substrate with centre-centre separation equal to a whole number of wavelengths. The resonators on the other hand can be either one-port or twoport devices, each having either one or two IDTs deposited on a piezoelectric substrate, between a pair of reflection gratings [6]. In a resonator, the acoustic waves are launched

within a resonant cavity thus causing the signal to be reflected back to the generating IDTs [6]. Figure 2.1 shows both delay-line and resonator structures. Both these devices fall under the category of surface launched acoustic wave devices. These devices are called surface launched as the wave motion is produced by the transducers on the surface and also picked by the transducers on the same surface, even though bulk waves are propagating in the surface. Acoustic sensors require a piezoelectric medium for generation of waves. The wave emanation can be explained in terms of crystal reordering. In a piezoelectric crystal, at equilibrium, inherent crystal strain is balanced by the internal polarization force. When disturbed either by applying external electric field or stress, de-polarization waves are emitted acting as a restoring force to maintain equilibrium [16]. The magnitude of the force emitted is directly proportional to stress applied as well as crystal orientation and arrangement of ferroelectric domains, both functions of crystal morphology.

\subsection{Two-Port Saw Resonator}

A two-port SAW resonator consists of an input IDT, output IDT and end reflection gratings. These IDTs are constructed on piezoelectric material such as quartz, 
lithium niobate, lithium tantalite etc. The cut of the substrate and orientation of the IDTs with respect to crystallographic axis are chosen such that it undergoes periodic deformation in response to applied potential. The application of electrical signal across a pair of interdigital transducers causes the piezoelectric material to be strained thus generating acoustic waves. The input IDT converts the applied electrical signal to mechanical acoustic waves. These waves travel across the surface towards the output IDT. The output IDT converts these mechanical SAW vibrations back to electrical voltage.

(A)

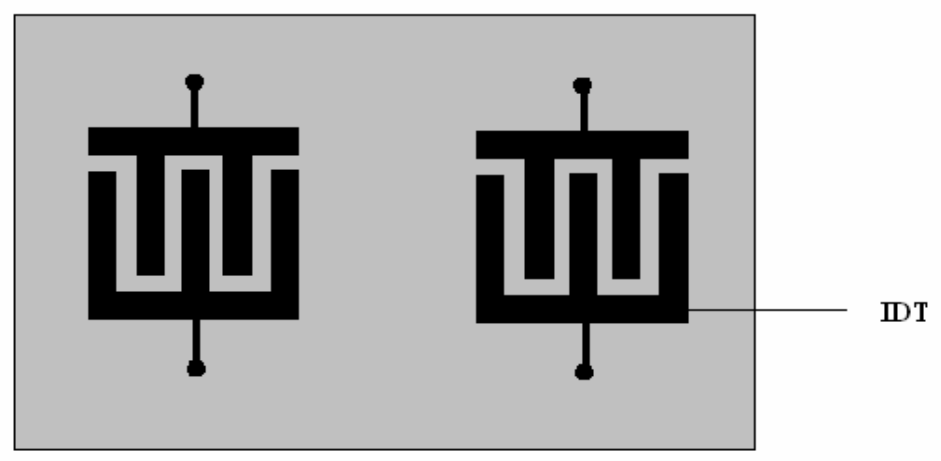

(B)

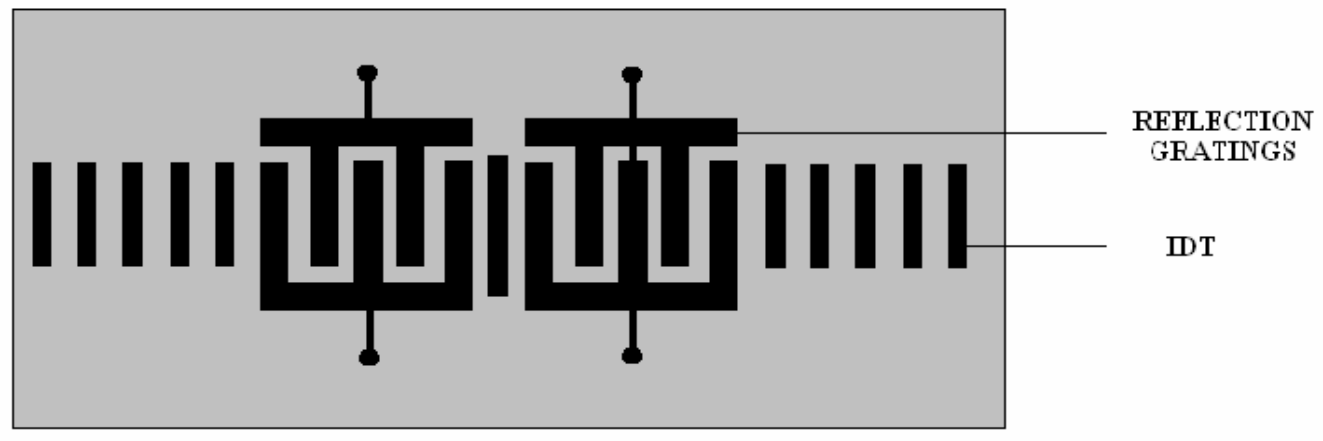

(C)

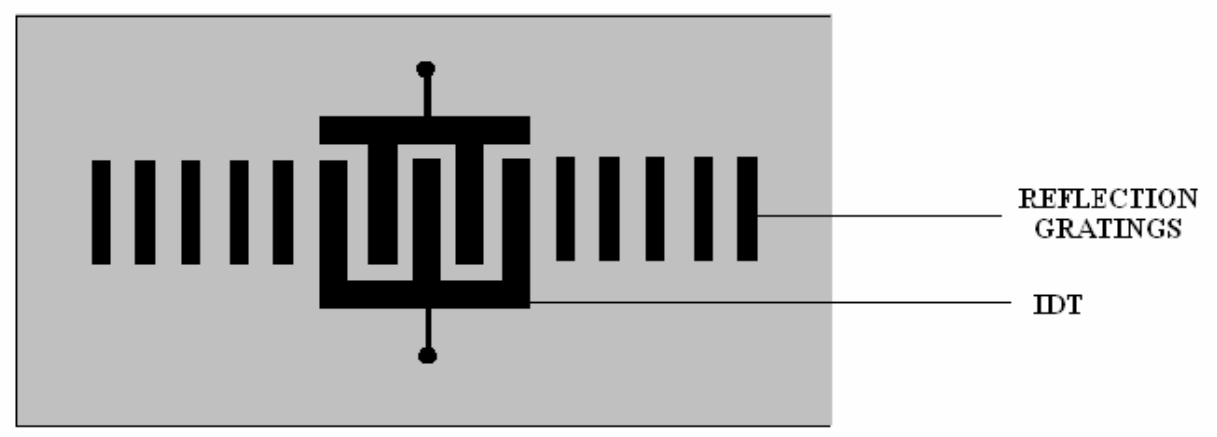

Figure 2.1. (A) Delay-Line (B) Two-Port SAW Resonator (C) Single-Port SAW Resonator 
In a two-port SAW resonators there are equal finger numbers at both the input and output IDT, with finger width equal to finger spacing. The equivalent circuit is shown in Figure 2.2 [15]. In the Figure 2.2 the shunt capacitor $\mathrm{C}_{\mathrm{T}}$ represents IDT capacitance while elements $L_{r}, C_{r}, R_{r}$ represent equivalent motional parameters for series resonance

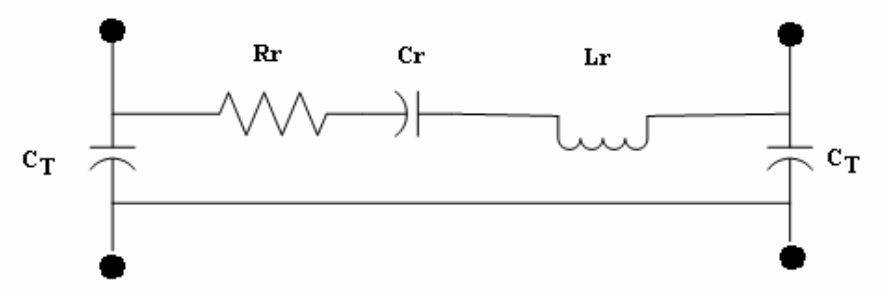

(A)

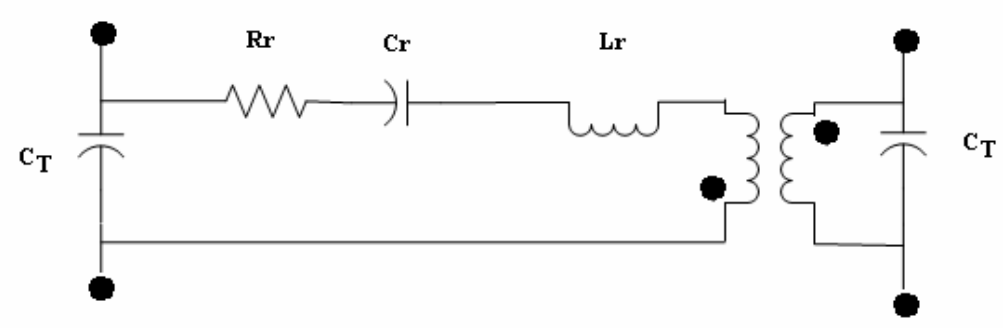

(B)

Figure 2.2. (A) Lumped Equivalent Circuit for $0^{\circ}$ Phase Shift between Input/Output IDT's at Resonance. (B) Ideal 1:-1 Transformer Included for $180^{\circ}$ Phase Shift at Resonance

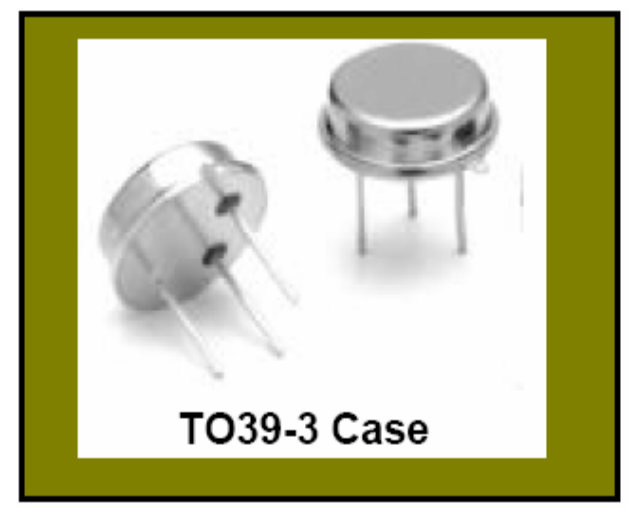

Figure 2.3. Two-Port SAW Resonator 
condition. The SAW resonator used for the experiment was RP 1239, 315 MHZ. A twoport RP 1239 SAW resonator consists of an input and output port. It is a three pin device having one pin for input, one for output and third pin is common and serves as device ground as shown in Figure 2.3 \{SAW resonator 1239).

\subsection{Operation}

When an alternating voltage is applied across the IDT, the substrate undergoes periodic deformation. This causes an acoustic wave to be launched propagating away from the IDT in both directions and perpendicular to the IDT fingers. The wavelength is determined by the IDT geometry and the spacing " $\mathrm{d}$ ' between the fingers as given by equation 1.

$\lambda=4 \mathrm{~d}$

The acoustic wave propagation velocity is determined by the acoustic mode, orientation and the type of substrate. This acoustic wave velocity is related to fundamental resonant frequency is given by equation 2 .

$f_{o}=\frac{v_{o}}{\lambda}$

Where

$f_{o}$ is wave frequency

$v_{o}$ is acoustic wave propagation velocity

$\lambda$ is wavelength

For any perturbation mechanism the velocity change can be measured as a change in resonant frequency, since the wavelength from equation 2 is seen to be fixed by the geometry of the device. The SAW device is highly sensitive to perturbation arising from 
many different mechanisms such as changes in mass density, elastic stiffness coefficients, dielectric property of film, electric conductivity [6] give by equation 3 . The variation of these characteristics causes the variation in SAW wave velocity " $v_{o}$ ". Variation of wave velocity is directly related to the changes in fundamental resonant frequency of the device. By using different perturbation approach and appropriate boundary condition it is possible to get the fractional changes in velocities which are related to frequency. The commonly used perturbation mechanisms are mass loading and acousto-electric mechanism.

$$
\frac{\Delta v}{v_{o}}=\frac{1}{v_{o}}\left[\frac{\partial v}{\partial m} \Delta m+\frac{\partial v}{\partial c} \Delta c+\frac{\partial v}{\partial \varepsilon} \Delta \varepsilon+\frac{\partial v}{\partial \sigma} \Delta \sigma+\frac{\partial v}{\partial T} \Delta T+\frac{\partial v}{\partial p} \Delta p+\ldots . .\right]
$$

$$
\begin{aligned}
& \text { Where, } \\
& \mathrm{m} \text { is mass } \\
& \mathrm{c} \text { is stiffness coefficient } \\
& \varepsilon \text { is dielectric constant } \\
& \sigma \text { is surface conductivity } \\
& \mathrm{T} \text { is temperature } \\
& \mathrm{P} \text { is pressure. }
\end{aligned}
$$

\subsection{Saw Preturbation Mechanisms}

In a surface acoustic wave device, the entire energy of surface waves is concentrated just at the surface. This makes the SAW devices highly sensitive to perturbations arising from many different physical parameters. Hence, the wave parameters such as velocity, frequency, amplitude and phase in an acoustic device are very sensitive to the immediate environment. Unlike other sensing mechanisms, it is more of a surface phenomenon. An incremental variation in the surface has pronounced effect on the wave parameters. The waves are hence easily and effectively affected by a medium placed along the path on the piezoelectric surface. The propagation of waves due 
to the presence of this medium is slightly disturbed. The amount of disturbance is dependent on thickness of the layer, its density and electrical conductivity. When gas molecules come in contact with this medium usually a selective absorbent film, an interaction occurs which causes the further disturbance of waves. The fundamental physical and chemical properties of these analytes are directly related to the wave disturbance which makes acoustic sensing more reliable scheme of detection.

\subsection{Saw Mass Loading}

One of the most common interactions between SAW device and surface film is due to mass loading. This occurs due to changes in mass deposited on device surface. This causes the wave velocity to decrease. The degree of effect produced is derived from energy consideration [16].The movement of wave along the surface having a thin or rigid film causes an increase in kinetic energy density $U_{k}[16]$. This changes in kinetic energy per area of surface is given by

$$
\Delta U_{k}=\frac{\rho_{s}}{4}\left(V_{x o}^{2}+V_{y o}^{2}+V_{z o}^{2}\right)
$$

Where $\mathrm{V}_{\mathrm{xo}}, \mathrm{V}_{\mathrm{yo}}$ and $\mathrm{V}_{\mathrm{zo}}$ the SAW particle velocity and $\rho_{\mathrm{s}}$ is surface mass density. The particle velocities are related to a displacement $u$ by $v_{i}=j w u_{i}$. The power density $P$ (power/area) carried by a wave can be related to wave energy $U$ (energy/volume) stored in a lossless medium as

$P=U v$

In a lossless medium $\mathrm{P}$ is constant. Differentiating (7) would yield

$$
\frac{\Delta u}{u_{o}}=\frac{-\Delta v}{v_{o}}
$$


Combining equation (6), (7) and (8)

$$
\frac{\Delta v}{v_{o}}=\frac{-\omega v_{o} \rho_{s}}{4}\left(\frac{V_{x o}^{2}}{\omega p}+\frac{V_{y o}^{2}}{\omega p}+\frac{V_{z o}^{2}}{\omega p}\right)
$$

Since the entire wave energy is concentrated near the surface, as operating frequency increases, surface particle velocities increase in proportion to $(P \omega)^{1 / 2}$. Thus, the quantity in equation 6 is independent of wave amplitude and is dependent only on substrate material.

$$
\left(\frac{V_{x o}^{2}}{\omega p}+\frac{V_{y o}^{2}}{\omega p}+\frac{V_{z o}^{2}}{\omega p}\right)
$$

Combining all substrate dependent parameters, the mass induced change in surface velocity is given by

$$
\frac{\Delta v}{v_{o}}=-c_{m} f_{o} \rho_{s}
$$

Where mass sensitivity factor $c_{m}$, is given by

$$
C_{m}=\frac{\pi v_{o}}{2}\left(\frac{V_{x o}^{2}}{\omega p}+\frac{V_{y o}^{2}}{\omega p}+\frac{V_{z o}^{2}}{\omega p}\right)
$$

From equation 11 we can see that the mass loading results in fractional changes in velocity. These fractional changes in velocity can be tracked by fractional changes in frequency. 


$$
\frac{\Delta v}{v_{o}}=\frac{\Delta f}{f_{o}}
$$

These fractional changes in frequency are tracked using an oscillator circuit thus tracking sensor response.

\subsection{Saw Acousto-electric Effect}

The acousto-electric effect in SAW arises from generation of a layer of bound charges at the surface accompanying the mechanical wave. The deposition of a conductive film causes the redistribution of charge carriers. This redistribution compensates the layer of bound charges developed due to passing surface wave. Figure 2.4 [16] shows the equivalent circuit model for SAW acousto-electric response.

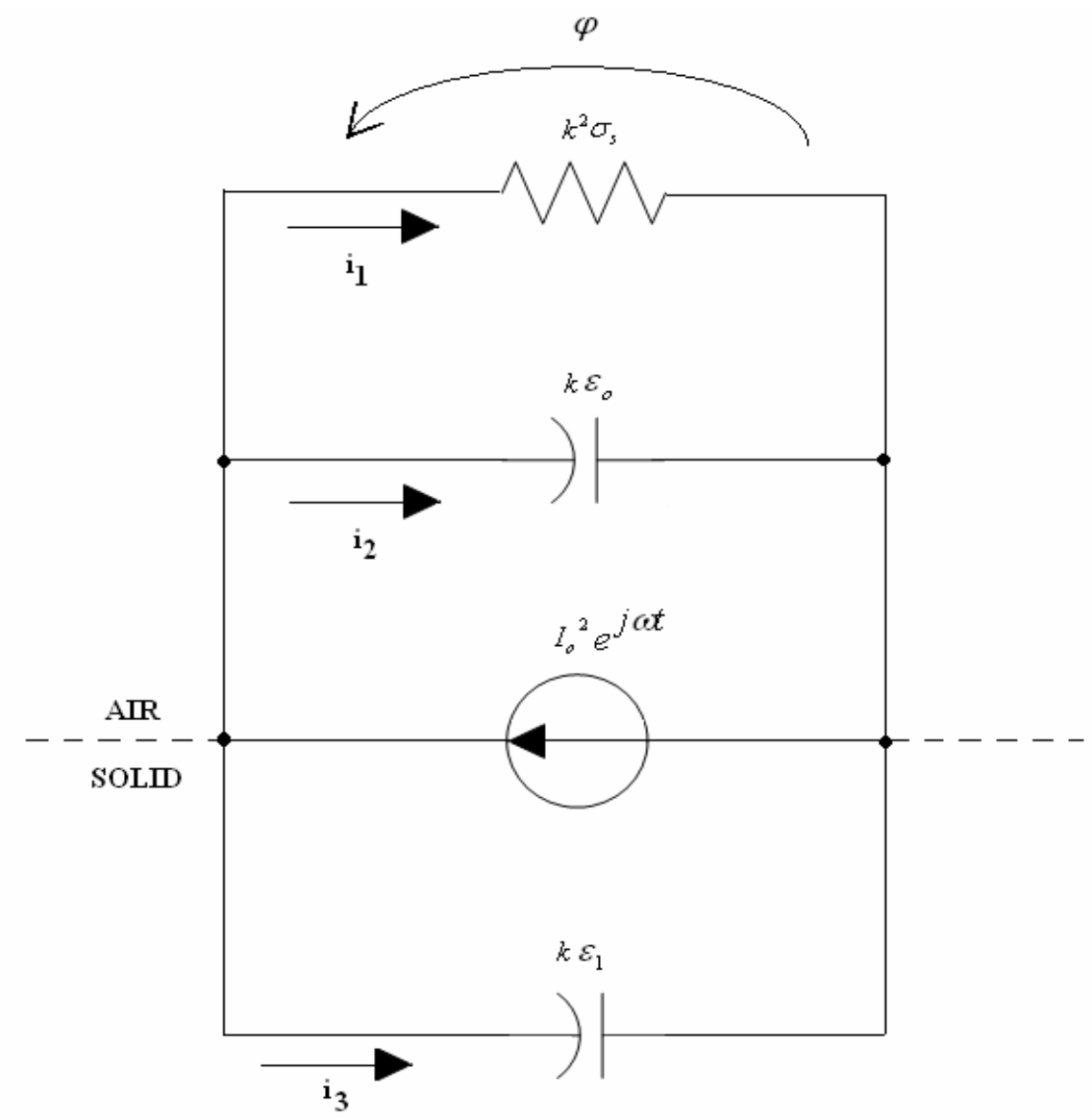

Figure 2.4. Equivalent Circuit Model Describing the Interaction between SAW and

Charge Carriers on Film Overlay 
The current generated per unit area of surface, $I_{o}$, is given as

$$
I_{o}^{2}=2 K^{2} \omega k^{2}\left(\varepsilon_{o}+\varepsilon_{s}\right) P
$$

Where

$K^{2}$ is Electro mechanical coupling coefficients

$\varepsilon_{o}$ and $\varepsilon_{s}$ are air and substrate dielectric permittivity

$k$ is acoustic wave number

$P$ is power density

Displacement currents generated in the substrate and air arise from capacitances of $s k \varepsilon_{o}$ and $k \varepsilon_{s}$, respectively. Shunt conductance $k \sigma^{2}$ accounts for the conduction currents in the film overlay. To study the effect of velocity and attenuation arising from SAW film acoustoelectric coupling two cases are considered.

1. Without conductive film - In the absence of conductive film the energy generated by the wave gets stored in the evanescent electric field. The power flow in this case is given as,

$$
P_{T 1}=\frac{I_{o}{ }^{2}}{2 j \omega k\left(\varepsilon_{o}+\varepsilon_{s}\right)}
$$

2. With the conductive film, the power flow becomes

$$
P_{T 2}=\frac{I_{o}{ }^{2}}{2\left[k^{2} \sigma_{s}+j \omega k\left(\varepsilon_{o}+\varepsilon_{s}\right)\right]}
$$


The overall response due to acousto-electric is the difference in two powers given by

$$
P_{T}=P_{T 2}-P_{T 1}=\frac{I_{o}^{2} k^{2} \sigma_{s}}{2\left[k^{2} \sigma_{s}+j \omega k C_{s}\right]}
$$

Where $C_{s}=\varepsilon_{o}+\varepsilon_{s}$

Putting equation (14) in (17) gives the complex power flow, $\mathrm{P}_{\mathrm{T}}$

The splitting of real and imaginary parts of power results in separating the effect of fractional changes in velocity and attenuation.

$$
\begin{aligned}
& \frac{\Delta v}{v_{o}}=-\frac{K^{2}}{2} \frac{\sigma_{s}^{2}}{\sigma_{s}^{2}+\left(v_{o} c_{s}\right)^{2}} \\
& \frac{\Delta \alpha}{k}=-\frac{K^{2}}{2} \frac{\sigma_{s} c_{S} v_{o}}{\sigma_{s}^{2}+\left(v_{o} c_{s}\right)^{2}}
\end{aligned}
$$

The magnitude of acousto-electric response is proportional to $K^{2}$, thus being dependent on substrate.

\subsection{Saw Sensor Operation}

The SAW device can be perturbed by a number of mechanisms as described in equation 3. This interaction occurring due to different mechanisms such as changes in mass density, elastic stiffness coefficients, dielectric property of film, electric conductivity causes the variation in SAW wave velocity " $V_{o}$ ". This variation in $V_{o}$ produces a phase shift between the applied and the received signals thus causing the frequency of minimum impedance to be shifted away from resonant frequency " $f$ '. Further, dampening of the acoustic waves may also result in decrease of amplitude of the received signals. Thus, perturbation can result in both phase and attenuation change [6]. 
These changes are usually measured by tracking changes in frequency, phase, attenuation or electrical impedance. Two methods are used to track these changes in the device:

1. The device either becomes a part of a frequency controlling element of an oscillator circuit

2. An external signal may be applied to the device with its phase and amplitude compared to the reflected or transmitted signal

The former is more commonly used as it's simpler and equipments required are cheaper. The other method is generally used in understanding device characteristics or understanding perturbation response mechanisms. The oscillator approach was used to design the sensor and the design approach is described in detail in the oscillator section of chapter 3.

\section{8. $\quad$ Oscillator Based Measurement Method}

There are two common methods of designing oscillator circuit for SAW filters. One method is a feed-back loop method and other is a negative resistance method. The feedback method is more commonly used for SAW delay-lines and SAW two-port resonators as they provide better control. In order for the SAW resonator to oscillate two essential conditions have to be met

1. The overall gain in the loop must be one or higher( at least $0 \mathrm{~dB}$ )

2. The overall phase shift in the loop must be an integral of $2 \mathrm{n} \Pi$, where $\mathrm{n}$ is an integer

Once the above two conditions are satisfied, a positive feedback would occur through the closed loop causing the SAW device to oscillate at its resonant frequency. The point to be noted here is that the oscillations occur at a frequency where both these conditions are met, which may or may not be the resonant frequency of the device and may lie in the neighbor hood of the resonant frequency [6]. The basic block diagram for the oscillator circuit is shown in Figure 2.5. The RF amplifier is chosen high enough so that it can compensate for losses in the feedback loop and provide a gain of at least $0 \mathrm{~dB}$. The amplifiers should be selected carefully as there are usually limits on the maximum permissible input and output power without damage. The choice of filters is done so as to remove any unwanted harmonics and spurious oscillations. 


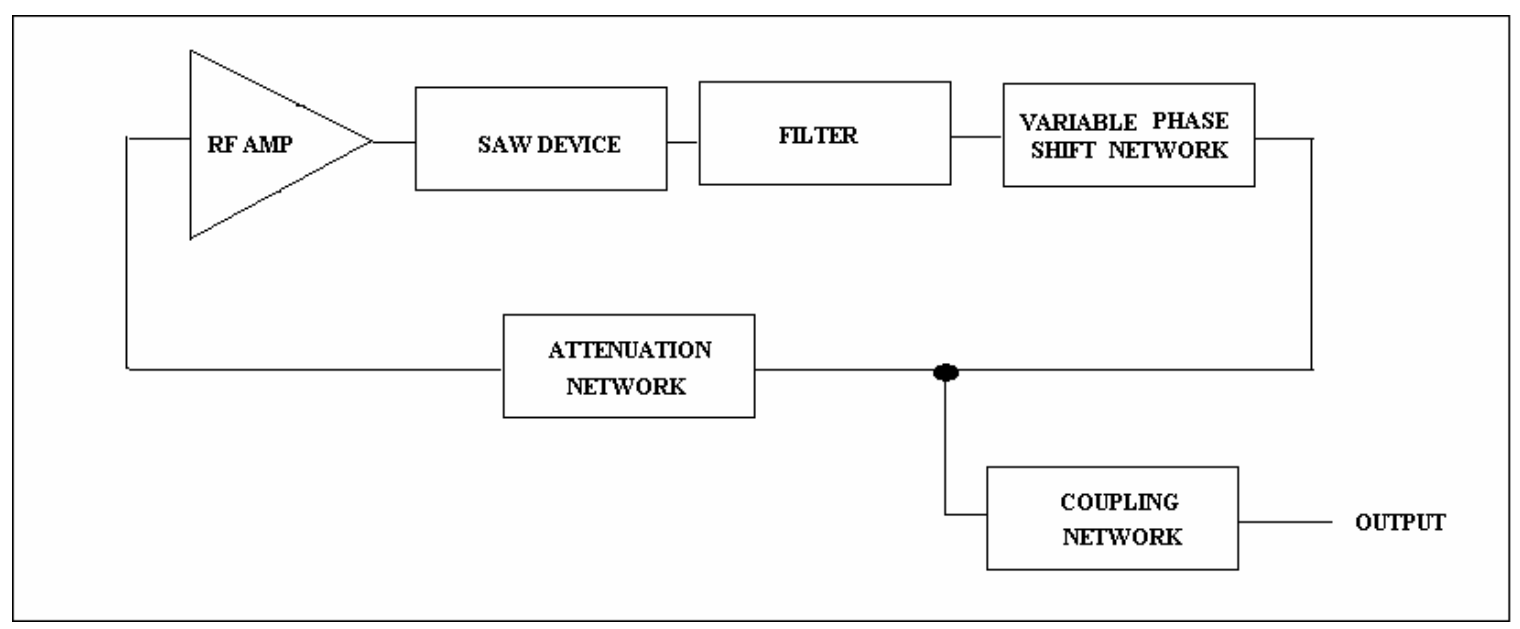

Figure 2.5. Oscillator Block Diagram

The phase shifters help in manually controlling the point of zero phase shift change and high gain to provide continuous and sustained oscillation. The attenuators are used to reduce the gain at the input of amplifier as they have a maximum permissible input level. One of the largest sources of error is the temperature and stability control of the amplifiers and voltage variable phase shifters. All these components rely on a stable DC power source and a temperature controlled environment.

\subsection{Type of Measurement}

SAW devices can be perturbed using a number of mechanisms as discussed earlier. These mechanisms depend on the interaction between the SAW device surface, the selective absorbent surface film and the gas. For a good interaction between the gas molecules and the selective absorbent surface film, there must be a means to transport these gas molecules to the surface. Also, these molecules must interact for some duration followed by which they should be transported back from the surface to outlet. Measurements could be made either statically or dynamically. Static measurements involve exposing the target analyte to a fixed amount of analyte in fixed volume of gas. These are usually used to investigate the properties of thin films deposited on the SAW device. The dynamic case involves the continuous transportation of carrier across the surface. There are two methods of dynamic measurements, one involving fixed analyte 
concentration throughout the measurement and the other involving a variation of analyte concentration from zero to maximum and back to zero. For accurate measurements they require good control of temperature, and pressure along with proper RF shielding and electrical connections.

\subsection{Understanding Sensor Response}

The target analyte reaching the surface should be absorbed by the sensor surface having a selective absorbent film. The SAW response to the analyte is driven by kinetic phenomenon such as adsorption, desorption, absorption and diffusion. These responses can occur either by weak physical interactions $\left(\Delta \mathrm{H}<40 \mathrm{KJ} \mathrm{mol}^{-1}\right)$ or chemical bond formation $\left(\Delta \mathrm{H}=80-400 \mathrm{KJ} \mathrm{mol}^{-1}\right.$ approximately) [17]. Physical absorption or physisorption usually occurs under conditions that favor liquefaction. These interactions are by weak Van der Waals forces or hydrogen bonding which are easily reversible. In physisorption the temperature alters the rate of surface absorption or desorption. Chemical adsorption or chemisorptions is less reversible process occurring usually at lower temperature and higher pressures. Some examples include dissociative adsorption process found in metal surface oxide and hydride film formation, and bonding of water molecules to such surface [6]. Some interaction such as Bronsted-Lowry and Lewis Acid lie between the chemisorptions and physisorption. There are also some interactions that are driven by entropic consideration typically in case of highly non polar analytes and sensor coatings which include hydrophobic effects. The rates of absorption/desorption and the sensor response are typically dependent on temperature, film properties and the amount of analyte concentration. Also the available sensor surface and its density also affect the rates of absorption which in turn affect the sensor response. At any instant the vapor-liquid phase analyte concentration, $C_{V}$, would give rise to a corresponding sensor phase analyte concentration, $C_{s}$, generating the resulting response [6]. $C_{V}$ is usually less than $C_{s}$ unless the process in irreversible. 


\subsection{Film Preperation}

SAW coatings fulfill two important roles. They provide a mechanism for providing selectivity towards the target analyte and the mechanism for improving device sensitivity. This sensing film can be coated on the surface of a SAW device using a number of techniques. The choice of method depends on ease of using the technique and available instruments for carrying out the same. Techniques such as casting, painting, dip coating. spin coating and drop coating are commonly used for coating the surface. Film thickness can usually be monitored by observing the frequency shift produced on coating. To get good coating the SAW surface must be thoroughly cleaned [18]. Commonly used techniques such as sonicating in chloroform, washing with strong acid, or plasma cleaning could be used to clean the surface. The type of coating method adopted also effects the type of film obtained [19] [20] [21]. Some key techniques of coating are explained below.

Spin Coating: Solution is dropped on the rotating crystal and centrifuge force spreads the solution. Factors such as rotating rate, solution viscosity, and solution density, directly affect the film thickness and uniformity.

Spray Coating: An airbrush containing a solvent reservoir is drawn upon by a mild flow of carrier gas. The solvent is sprayed through a small nozzle. The type and amount of coating is dependent on the skill of the person using it. The material is sprayed till the desired coating is achieved.

Drop Coating: The solution of a required volume is dropped on the surface using a pipette. The solvent is allowed to evaporate by itself or with the help of gentle heating. This is a simple method for achieving desired thickness.

\subsection{Gas Delivery}

There are two types of flow devices, volume flow devices and mass flow devices. As the name suggests volume flow devices tend to control the volume of gas flow. Volumetric flow $(\mathrm{Q})$ measurement techniques require separate measurements of pressure (P) and temperature (T) to calculate density and mass flow (m). These measurements are hence less reliable as they are easily affected by temperature and pressure changes which 
affect the density of gas. Mass Flow devices on the other hand are not affected by changes in pressure and temperature as they regulate molecular flow. Hence they are more reliable, repeatable and a more accurate method for delivering the analyte or the required material at a desired rate. Mass flow meters are usually calibrated for a specific gas or flow range to provide direct readout. They can be used for different gases by applying appropriate conversion factors calculated from thermodynamic considerations. Mass flow devices such as thermal mass flow meters (MFMs) and mass flow controllers (MFCs) are commonly used for delivery of gas. Table 2.1 shows the measurement techniques used for some volumetric and mass flow measuring devices.

Table 2.1. Measurement Techniques Used by Different Gas Delivery Systems

\begin{tabular}{|c|c|c|}
\hline Flow meter & Measurement Technique & Parameters Required \\
\hline Turbine Meter & Q & Need P,T \\
\hline Positive Displacement & Q & Need P,T \\
\hline Orifice Meter & Q & Need P,T \\
\hline Rotameter & Q & Need P,T \\
\hline Thermal MFC/MFM & M & Measures Mass Directly \\
\hline
\end{tabular}

\subsection{Mass Flow Controller Operation}

The mass flow controllers are flow measurement techniques commonly used for regulating the flow of gas. The gases that flow in the lines are divided into two parts while entering the MFCs. Most of the gas (m2) flows through the bypass thus creating a pressure drop causing a small part of the flow $(\mathrm{m} 1)$ to go through the sensor tube as shown in Figure 2.6. The sensor tube has two resistance coils, causing a fixed amount of heat flow into the tube. Heat gets transferred from the tube to the gas molecules. Heat transfer between these elements results from interaction with the molecules of the flowing gas independent of pressure or temperature fluctuations. The gas carries this heat from the upstream coil to the downstream coil. The downstream coil has a higher resistance and is maintained at a higher temperature as compared to the upstream coil. These resistors form legs of a Wheatstone bridge whose output voltage is directly 
calibrated to calculate the mass of gas flowing in the tube. As the gas from bypass and sensor tube leaves the MFC they have to go through a servo controlled Valve. This valve could be electromagnet, where the current in the electromagnet modulates the orifice opening thus maintaining a fixed mass of gas flow. Built-in PID electronics allows the device to maintain continuous proportional control by comparing the measured sensor signal to the commanded flow rate. These valves could be normally open ones or closed configurations. Most thermal MFMs and MFCs are specified at $\pm 1 \%$ of full-scale accuracy and $\pm 0.25 \%$ of full-scale repeatability.

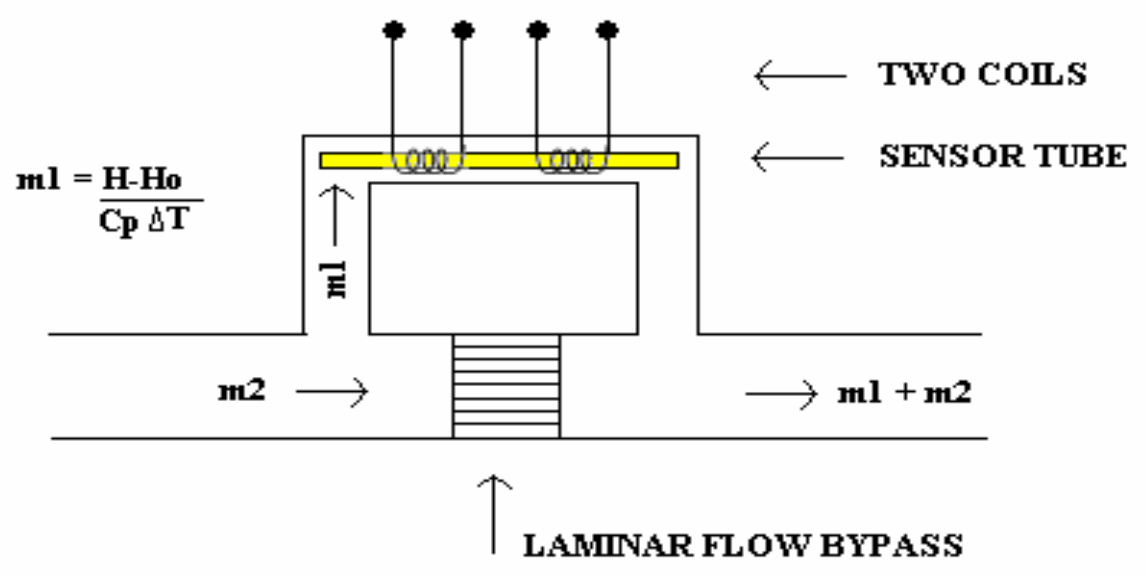

Figure 2.6. The Mass Flow Controller

\subsection{Saw Housing}

The manner in which SAW device is mounted and coupled to the sample handling system determines the type of SAW housing to be designed. The key consideration while designing SAW housing are precise temperature, pressure control, satisfactory electrical connections and chemical stability of housing material. Some material such as Brass, Aluminum, Stainless Steel and PTFE are commonly used to designing housing. Though brass and aluminum provide good electrical shielding, good temperature stability and are easy to machine, they however suffer from corrosion by chemicals used in the housing. PTFE, though is a good material free from corrosion due to chemical attack, suffers from problems of machining as they tend to creep and deform due to applied pressure or stress. 
Stainless steel is a good material of choice as it is not susceptible to problems discussed previously.

\subsection{Sensing Layer}

For successful detection of target analyte the SAW device must be coated with a suitable sensing film. The sensing film chosen should provide selectivity and enhanced sensitivity to the target analyte which is a key requirement for a gas sensor. Two nanomaterials sensing layer chosen in this study were Carbon Nanotubes and Tobacco Mosaic Virus.

\subsection{Carbon Nanotubes}

Carbon Nanotubes (CNTs) are thin, cylindrical structures made of carbon similar to honey comb lattice. Out of the many polymorphs of carbon they tend to resemble graphite. Though they are similar to buckyballs as both are made of graphite there exits an enormous structural difference. They structural difference arises from the fact that the buckyballs are graphite sheets rolled into balls, while the CNT are graphite sheets rolled in the form of cylinder. This structural difference gives remarkable electronic and mechanical properties to carbon nanotubes. They are known to have a Young's modulus of over 1 tera Pascal and estimated tensile strength of 200 giga Pascal. Figure 2.7 shows the SEM image of Carbon Nanotubes [22]. The carbon nanotubes can be manufactured in two types:

1. Single walled carbon nanotube (SWNT)

2. Multi walled carbon nanotubes (MWNT)

Each of them has distinct material properties making them suitable for different applications. 


\subsection{Single Walled Nanotubes (SWNTs)}

SWNTs are sheet of graphite rolled in the form of cylinder. They usually have diameters in the range of $1-2 \mathrm{~nm}$ with $2-5$ microns in length. Figure 2.8 shows a microscopic image of SWNTs [23]. They can be more easily twisted, flattened and bent into small circles around sharp bends without breaking, unlike MWNTs. They can be either metallic or semi-conducting. They also have very high tensile strength which is about 100 times of steel at a size that is $1 / 6$ of it. There are 3 main configurations for SWNTs.

1. Armchair

2. Zigzag

3. Chiral

Figure 2.9 [24] shows all the three configuration of SWNTs. Each of them differs, in the manner in which they are rolled up along the diameter. This difference gives them different properties. These properties can be determined using a vector called chiral vector. This is given by

$\mathrm{Ch}=\mathrm{na}_{1}+\mathrm{na}_{2}$

Where, $\mathrm{na}_{1}$ and $\mathrm{na}_{2}$ are unit vectors in 2 - dimensional hexagonal lattice and $\mathrm{n}, \mathrm{m}$ are integers. Depending on the value of chiral vector and chiral angle (which is the angle between $\mathrm{Ch}$ and $\mathrm{na}_{1}$ ) they can be metallic and semiconductor. Armchair varieties are usually metallic having $\mathrm{n}=\mathrm{m}$ with $0^{\circ}$ chiral angle. Chiral type SWNTs has a chiral angle anywhere between $0-30^{\circ}$, while the zigzag variety has a chiral angle of $30^{\circ}$.

\subsection{Multi Walled Nanotubes (MWNTs)}

Multi Walled Nanotubes (MWNT) can be considered as a collection of concentric SWNTs as shown in Figure 2.10 [24]. They are usually 2-20nm in diameter with $100 \mathrm{~nm}$ or more in length. They can have anywhere between 5-20 graphite layers. Due to layered structure they usually have a mix of semi-conducting and metallic properties. A typical 
MWNT is shown in Figure 2.10. Although it is easier to produce significant quantities of MWCNTs than SWNTs, their structures are less well understood than single-wall carbon nanotubes because of their greater complexity and variety. Today, both SWNT and MWNT are being extensively used in the production of high strength composite materials, sensors, optical devices, hydrogen fuel cells with the type of device determining the type of nanotube desired.

\subsection{Tobacco Mosaic Virus (TMV)}

Tobacco mosaic virus is known to cause plant disease in more than 150 types of herbaceous, dicotyledonous plants including many vegetables, flowers, and weeds [25]. These viruses don't kill the crop but lower their yield and quality of the crop. Their infection on plants is usually characterized by intermingled patches of normal and light green or yellowish colors on the leaves [26]. They are about TMV is $300 \mathrm{~nm}$ long, $18 \mathrm{~nm}$ in diameter and with a $4 \mathrm{~nm}$ in diameter axial channel $[27,28]$. They have been previously coated with metals, silica or semi-conducting materials to form nanorod assemblies. Figure 2.11 [28] and Figure 2.12 [29] shows the TEM image TMV tube and of a TMV Pd coated TMV tubes respectively.

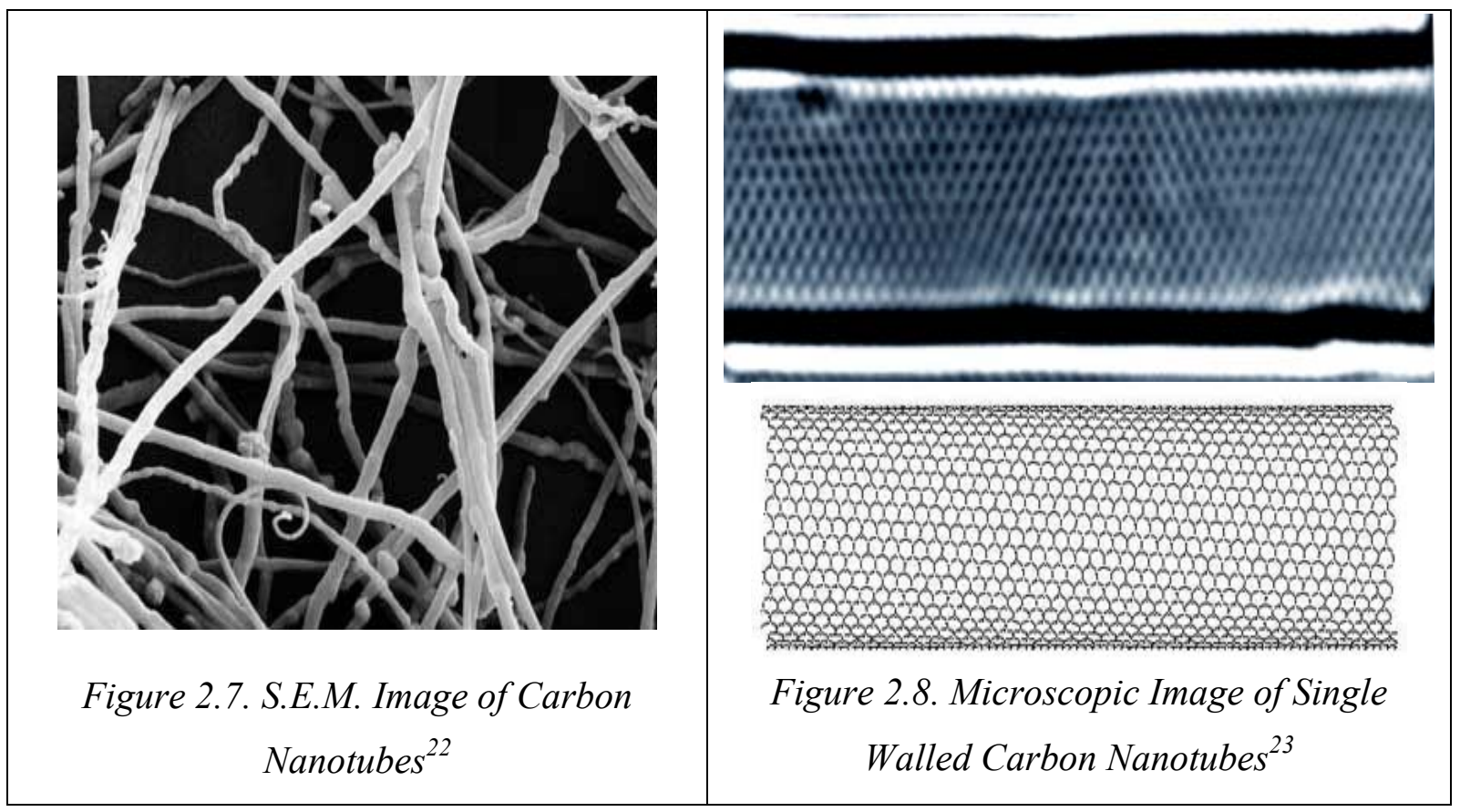




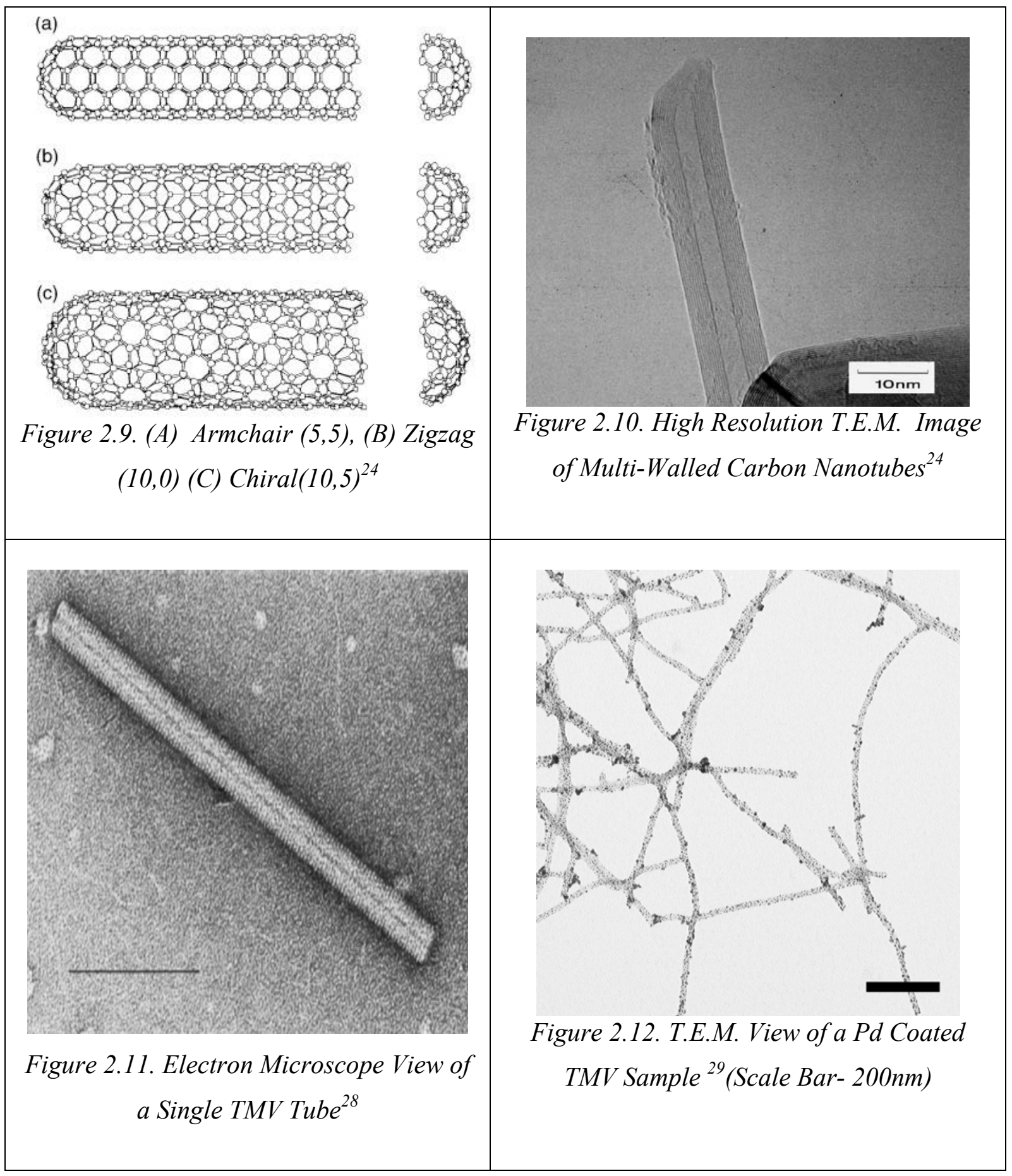




\section{CHAPTER 3: EXPERIMENTAL SETUP}

\subsection{Gas Dilution System}

An in-house developed custom-automated gas dilution system [30] was utilized for these experiments. The system was designed keeping in mind several factors. First there was a need for a controllable concentration analyte gas, and a purge gas. These conditions where met by using 4 MKS Type 1479A mass flow controllers (MFCs) connected to a MKS Type 247 4-channel readout. The four flow controllers have different maximum flow rates to allow for maximum accuracy and controllability across a wide range of gas concentrations from high $\mathrm{ppb}$ to pure while maintaining a steady flow rate, depending upon the concentration of the analyte gas cylinder concentration. The MFCs in this work were used to mix hydrogen with nitrogen to obtain different range of concentrations from 0 volume $\%$ to 5 volume $\%$.

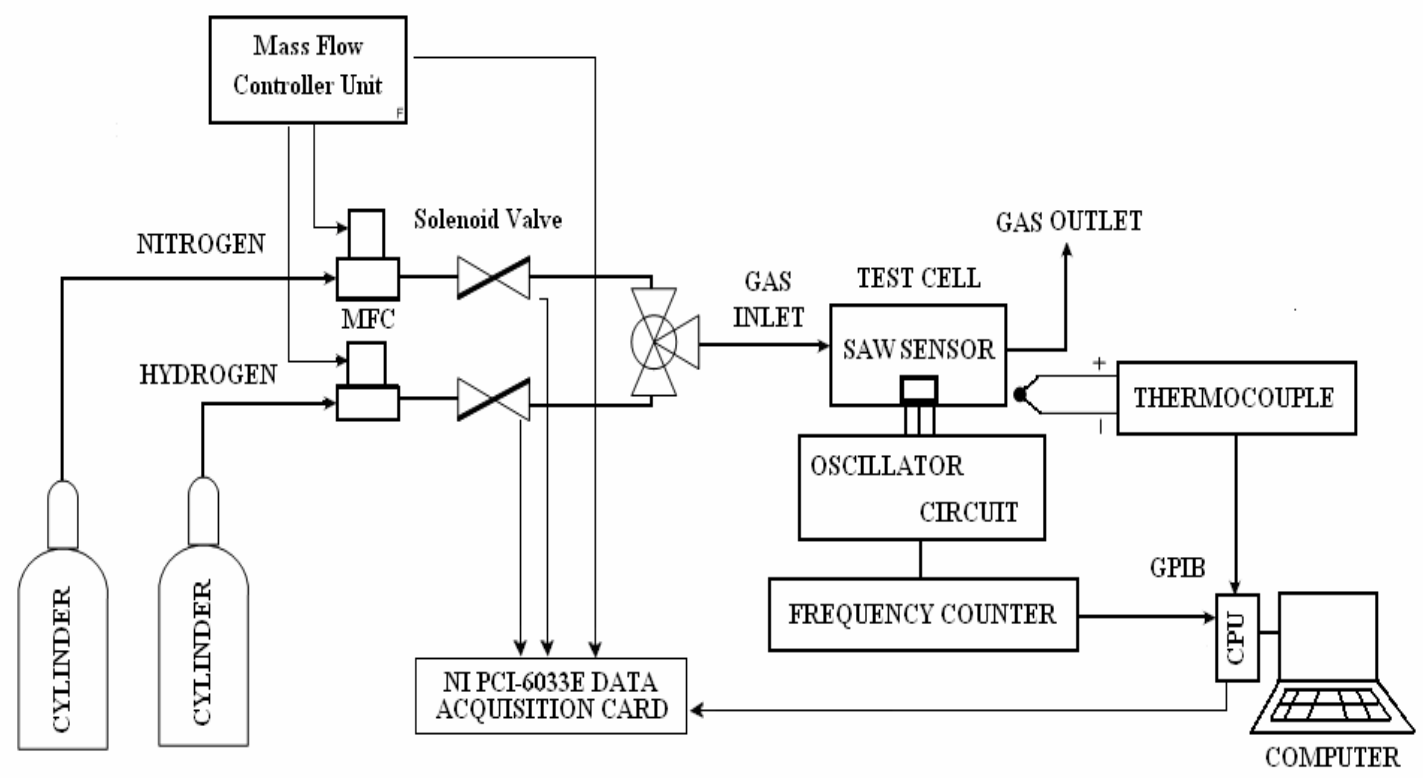

Figure 3.1. Mass Flow Controller Setup 
These gases pass through a set of solenoid valves which were selected in operation for the concentrations required. The gases flowing through the mass flow controllers are mixed in the mixing chamber with carrier gas. The gases are then passed through a temperature controlled cell. Stainless steel tubing is chosen to connect the cell and the solenoid valves while PTFE tubing is used between the cell and the exhaust. The selection of MFC, solenoids and the concentration is done using a LABVIEW (version 7) program through a National Instruments PCI Data Acquisition (DAQ) card. The fully automated system has a cycle time of approximately 5 seconds [19].

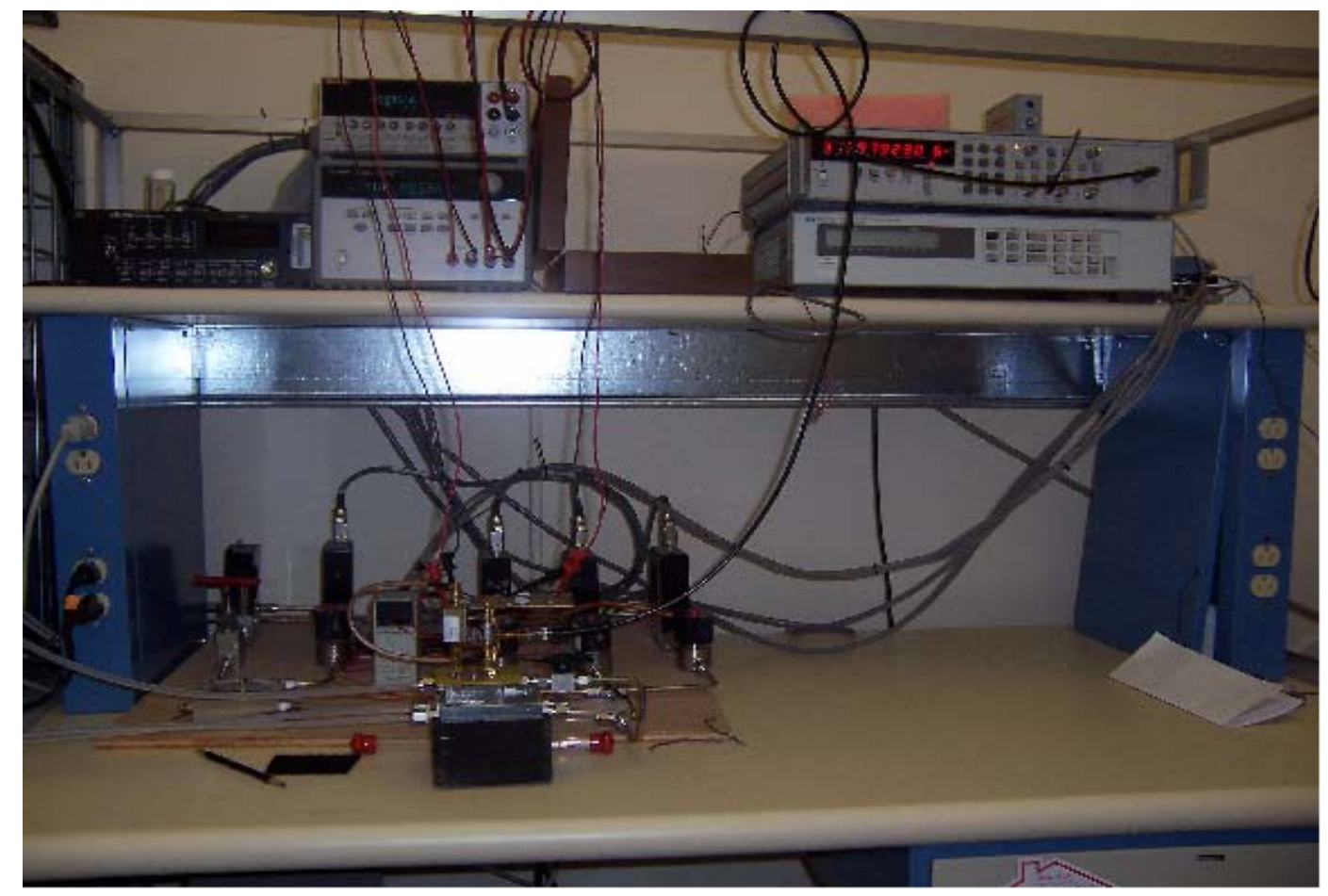

Figure 3.2. The Complete Experimental Setup

\subsection{Calibration of Mass Flow Controllers}

The gas flow controller has to be calibrated for it to detect the flow rates of different gases as they are generally calibrated for nitrogen. There are a set of scaling potentiometers on the rear panel of the mass flow controller readouts. These 10 turn potentiometers are used to adjust the full scale voltage signals from the MFC, which 
correspond to the flow rate, to a level that enables the digital panel meter to display the flow rate and set point directly, in sccm. The factor is called Scaling Control Factor.

Scaling Control Factor $=$ Gauge Factor $*$ Gas Correction Factor

The Gauge factor is the factory set value which scales the +5VDC output signal to the appropriate full scale range for the MFC, so that the panel meter reads 1000 counts.

Table 3.1. MFC Gauge Factor

\begin{tabular}{|l|l|}
\hline MFC Gauge Factors & Gauge Factor \\
\hline MFC Flow Range(sccm) & 100 \\
\hline $1,10,100,1000,10 \mathrm{~K}$ & 200 \\
\hline $2,20,200,2000,2 \mathrm{~K}$ & 50 \\
\hline $5,50,500,5000,5 \mathrm{~K}$ & \\
\hline
\end{tabular}

\section{Gas Correction factor (GCF)}

A gas correction factor is used to indicate he ratio of flow rates of different gases which will produce the same output voltage from mass flow controllers.

$\mathrm{GCF}_{\mathrm{X}}=(\underline{0.3106) *(\mathrm{~S})}$

$\left(\mathrm{d}_{\mathrm{x}}\right)\left(\mathrm{Cp} \mathrm{p}_{\mathrm{x}}\right)$

GCFx depends on specific heat, density, and molecular structure of gases.

$\mathrm{d}_{\mathrm{x}}$ is Standard density of gas , $\mathrm{g} / 1$ ( at $0^{\circ} \mathrm{c}$ and $760 \mathrm{~mm} \mathrm{Hg}$ )

$\mathrm{Cp}_{\mathrm{x}}$ is Specific heat of gas $\mathrm{x}, \mathrm{cal} / \mathrm{g}^{\mathrm{o}} \mathrm{C}$

0.3106 is (Standard density of nitrogen) (Specific heat capacity of nitrogen)

$\mathrm{S}$ is Molecular structure correction factor

Where $S$ equals:

1.030 for Monatomic gas

1.000 for Diatomic gas 
0.941 for Triatomic gas

0.880 for polyatomic gas

$G C F_{x}=\frac{0.3106 * \mathrm{~S}}{d_{x} * C p_{x}}$

Applying temperature correction factor as these values of gas correction factors were calculated at $0^{\circ}(273$ Kelvin).

Temperature corrected GCF $=G C F * \frac{T_{x}}{T_{s}}$

Where

$\mathrm{T}_{\mathrm{x}}=$ Reference temperature at $\left({ }^{\circ} \mathrm{K}\right)$

$\mathrm{T}_{\mathrm{s}}=273.15^{\circ} \mathrm{K}\left(0^{\circ} \mathrm{C}\right)$

For hydrogen,

$G C F_{x}=\frac{0.3106 * \mathrm{~S}}{d_{x} * C p_{x}}$

$\mathrm{GCF}_{\mathrm{x}}$ depends on specific heat, density, and molecular structure of gases.

$\mathrm{d}_{\mathrm{x}}=0.0899$

$\mathrm{Cp}_{\mathrm{x}}=0.3106$

$\mathrm{S}=1.000$ for Diatomic gas $\left(\mathrm{H}_{2}\right)$

$G C F_{x}=1.0$

Temperature corrected GCF $=G C F * \frac{T_{x}}{T_{s}}$

$\mathrm{T}_{\mathrm{x}}=297.15 \mathrm{~K}\left(24^{\circ} \mathrm{C}\right)$

$\mathrm{T}_{\mathrm{s}}=273 \mathrm{~K}\left(0^{\circ} \mathrm{C}\right)$

Temperature corrected GCF $=1.08$ 
Table 3.2. Scaling Factor for Hydrogen Based on Flow Rates

\begin{tabular}{|c|c|c|c|}
\hline $\begin{array}{c}\text { MFC Flow rate } \\
(\mathrm{sccm})\end{array}$ & GCF & Guage Factor & $\begin{array}{c}\text { SCF= GCF*Gauge } \\
\text { Factor }\end{array}$ \\
\hline 500 & 1.08 & 50 & 54.80 \\
\hline 10 & 1.08 & 100 & 109.70 \\
\hline 100 & 1.08 & 100 & 109.70 \\
\hline 2000 & 1.08 & 200 & 219.330 \\
\hline
\end{tabular}

\subsection{Test Cell}

The SAW device was housed in a cell made of Stainless Steel. The key considerations while designing the cell were precise control of temperature and chemical stability of housing material. Also, the cell volume was required to be small so as minimize the time required for the material to interact with the sensing material and thus achieve quicker stability. The flow should also be streamlined. Based on the requirements the cell design was designed in Microsoft Visio as shown in Figure 3.3.

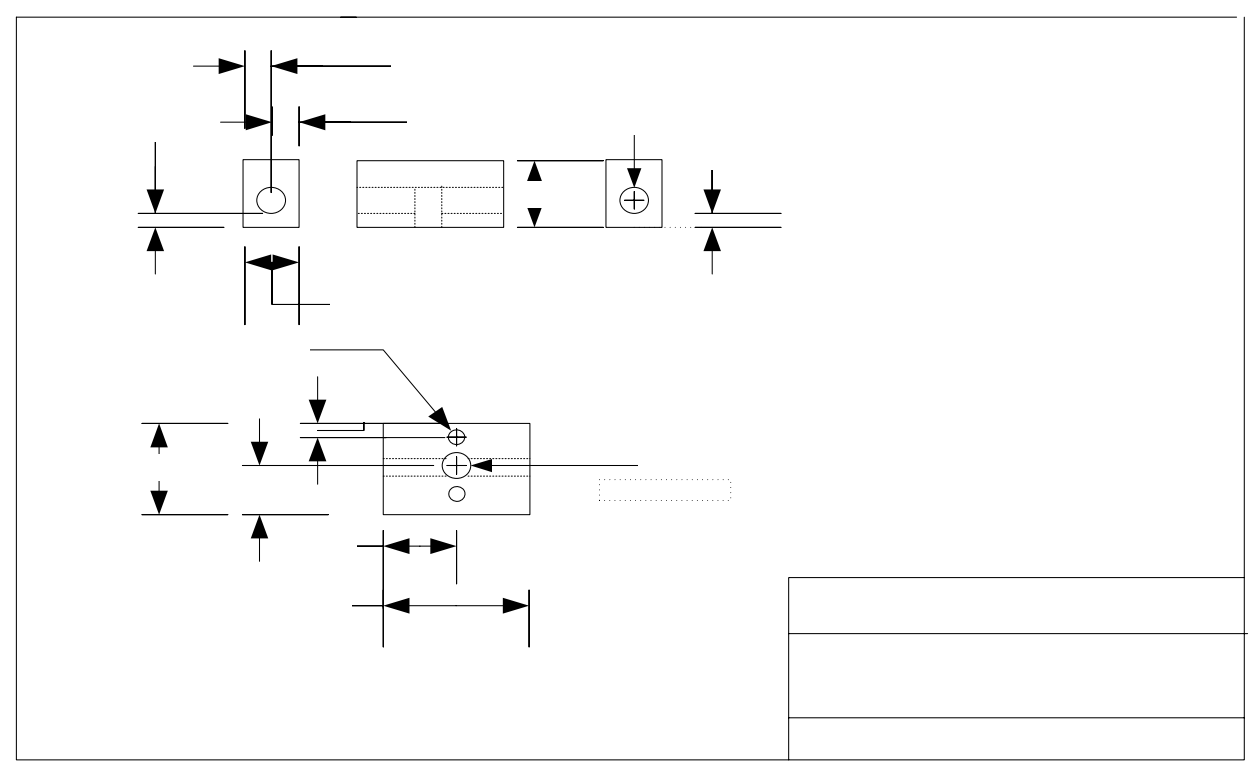

Figure 3.3. Schematic of the Cell Design 
The cell was made of $312 \mathrm{~L}$ stainless steel. Stainless steel was used as it does not creep or deform due to applied pressure. This is important as the PCB would be removed very often for changing the SAW device or increasing the coating on the surface of the device. The cell has an inlet and outlet for allowing the gases to and from the cell. The input of the cell was fed from the solenoids connected to the mass flow controllers while the output fed to exhaust. Connections between the cell and the solenoid connected to the MFC were achieved with help of National Pipe Taper (NPT) from Swagelok. Female connectors are commonly used on the tubing ends while male couplings on the casing. The inlet and outlet were threaded for connecting $1 / 4$ " NPT male thread connector capable of handling pressures up to 10000 psi. These male connectors were connected to the inlet and outlet lines using $1 / 4 "$

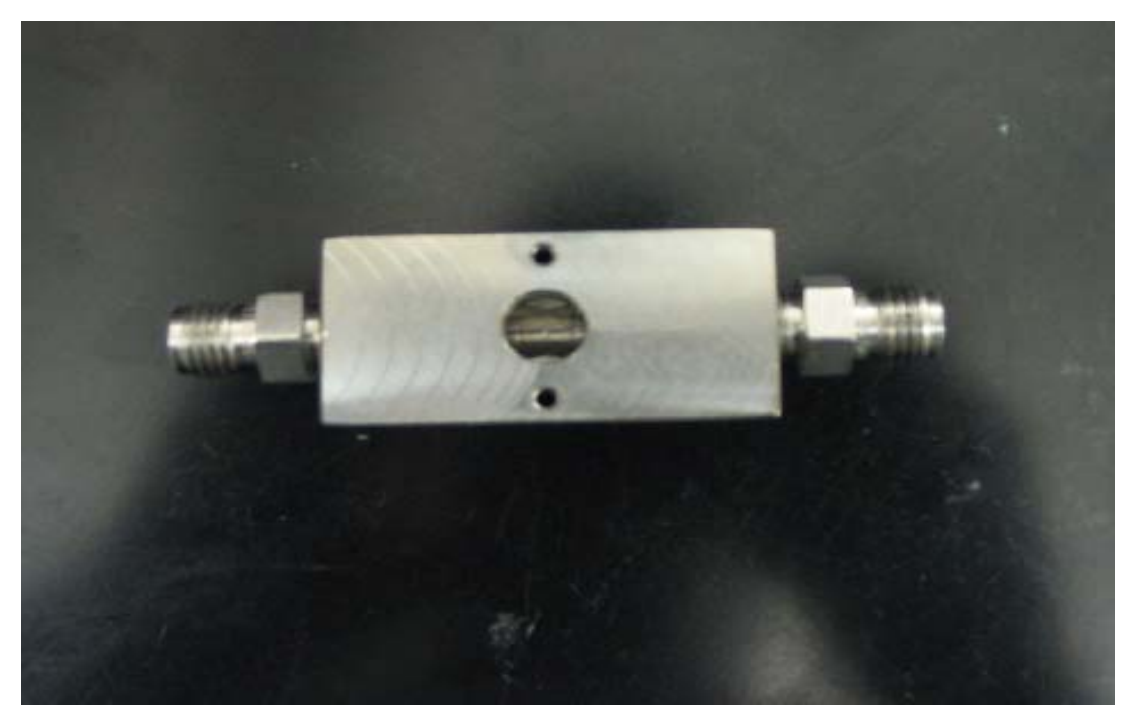

Figure 3.4. The Machined Stainless Steel Cell

female NPT threads, available at the tube endings. The cell has a small circular opening in the top to house the SAW resonator. The SAW resonator was supported on a printed circuit board. The printed circuit board supporting the resonator was tightly held on two the cell with help of two Allen screws. The machined cell is shown in Figure 3.4. 


\subsection{Saw Resonator - PCB Design}

A commercial two-port SAW resonator RP1239, 315MHZ was used to design the sensor. The two-port resonator consists of three terminals, one input, output and ground terminal that is common and serves as device ground. The commercial resonator is shown in Figure 2.3. The resonator was connected to the printed circuit board directly. The design of the printed circuit board was done in-house. Several key factors were kept in mind before designing the board. Since the SAW device operates at $315 \mathrm{MHz}$ it was ensured that the there were no bends or curves since RF effects could play a key role in changing the characteristics of the board, such as adding additional phase or changing the characteristic impedance of the board as was seen in one of the earlier PCB designs. This copper board to be etched was a commercial presensitized board. A presensitized board is a double sided copper board having positive photoresist precoated on both sides.

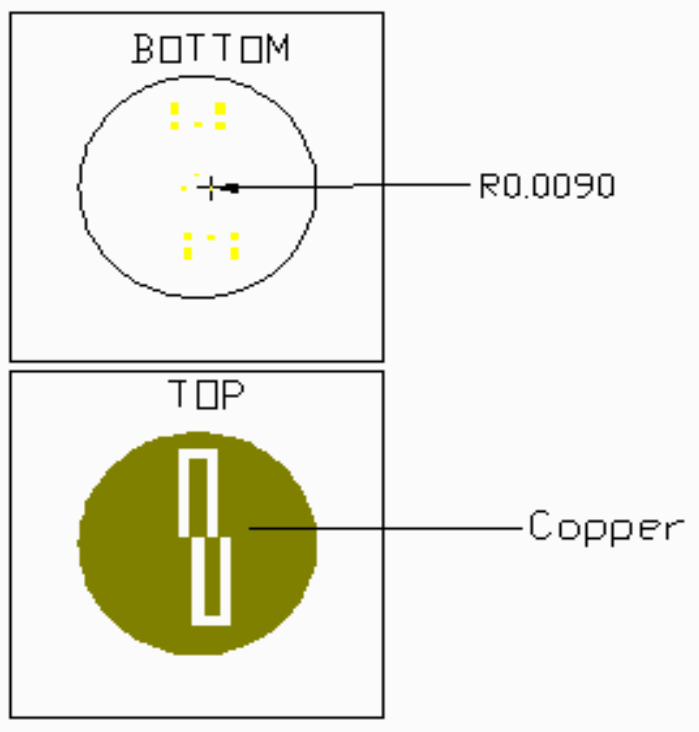

Figure 3.5. The Top and Bottom Surface of PCB Designed in AutoCAD. The Bottom Side is One Facing the Cell

The design of the board was made in AutoCAD as shown in Figure 3.5 keeping all the parameters in mind. This design was later transferred on a transparency acting as a mask to be used on a presensitized copper board. The process of development of board is described in detail in the Appendix A. The SAW device was directly soldered on the 
board to avoid any loose connections which were encountered while designing the board with sockets. The SAW device input and output were connected to the oscillator with help of SMA connector. The top and bottom views of the PCB design is shown in Figure 3.6 and Figure 3.7.

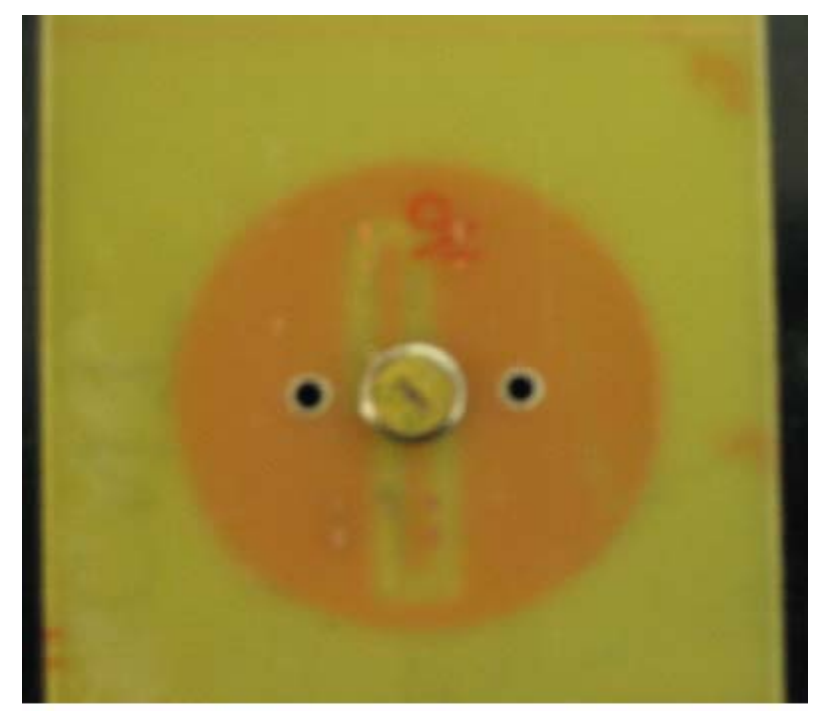

Figure 3.6. The Final PCB Design (Bottom)

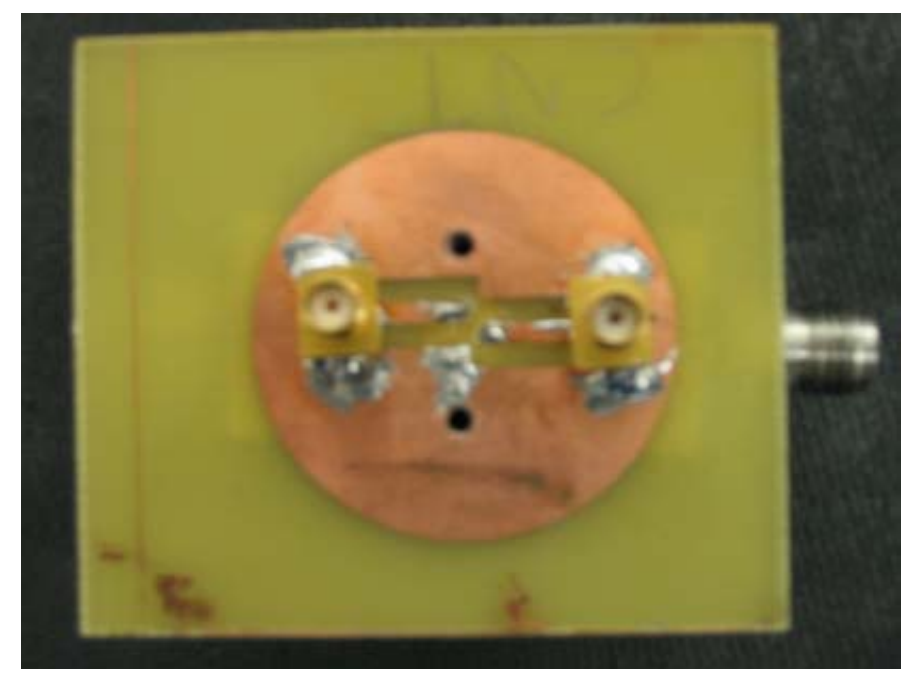

Figure 3.7. The Final PCB Design (Top)

\subsection{Oscillator Circuit}

A feedback loop oscillator circuit was designed with the SAW resonator as the frequency controlling element. The design of the feedback loop ensured that there was 
positive feedback through the loop providing an overall phase shift of $2 n \pi$, where $n$ is an integer, thus causing the SAW resonator to oscillate at its resonant frequency.

\subsubsection{Amplifier}

To provide the necessary gain for driving the SAW resonator a high gain, broad band monolithic, Minicircuits ZFL-500LN amplifier was chosen. The amplifier provided a $24 \mathrm{~dB}$ gain in $0.1-500 \mathrm{MHz}$ bandwidth. The input voltage driving the amplifier was $15 \mathrm{~V}$ with current of $60 \mathrm{~mA}$. The maximum input and output power was $+5 \mathrm{dBm}$. It provided a low noise Figure (N.F.) $<2.9 \mathrm{~dB}$ and $3^{\text {rd }}$ order intercept point of $14 \mathrm{~dB}$. The input and output VSWR was 1.5:1 and 1.6:1 respectively with input and output impedance of $50 \mathrm{ohm}$. The amplifier was chosen in a manner to have SMA connectors at the end. The output of the amplifier was connected to the input of the SAW resonator board.

\subsubsection{Filter}

The low pass VLF-320, filter was used in the oscillator circuit to avoid oscillation at spurious frequencies. The low pass filter was chosen to have a cutoff at $320 \mathrm{MHz}$ with a loss of less than $1 \mathrm{~dB}$ in the pass band. The filter was connected to the output of the SAW resonator board.

\subsubsection{Attenuator}

A set of fixed attenuator was chosen in order to reduce the overall gain in the circuit so as to prevent the maximum permissible input to the amplifier. MiniCircuits VAT series attenuators of 3,6,10 dB were used as and when required in the circuit depending on the amount of attenuation required. These fixed attenuators had SMA connector at both input and output.

\subsubsection{Phase Shifter}

To provide the required phase shift of $2 \mathrm{n} \Pi$, a phase shifter was introduced in the circuit. Two voltage variable MiniCircuits JSPHS-446, phase shifter were used in order 
to provide a minimum phase shift of $360^{\circ}$. The phase shifters used were surface mount devices.

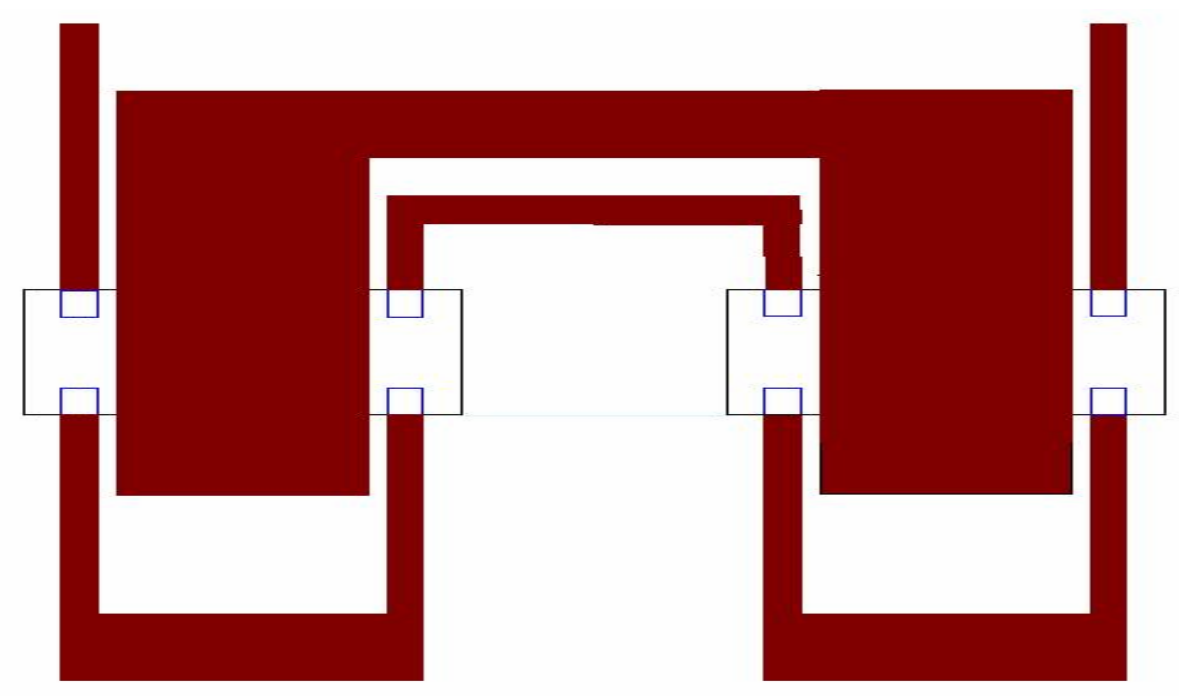

Figure 3.8. PCB Design for Two 180 Degree Phase Shifter Connected in Series

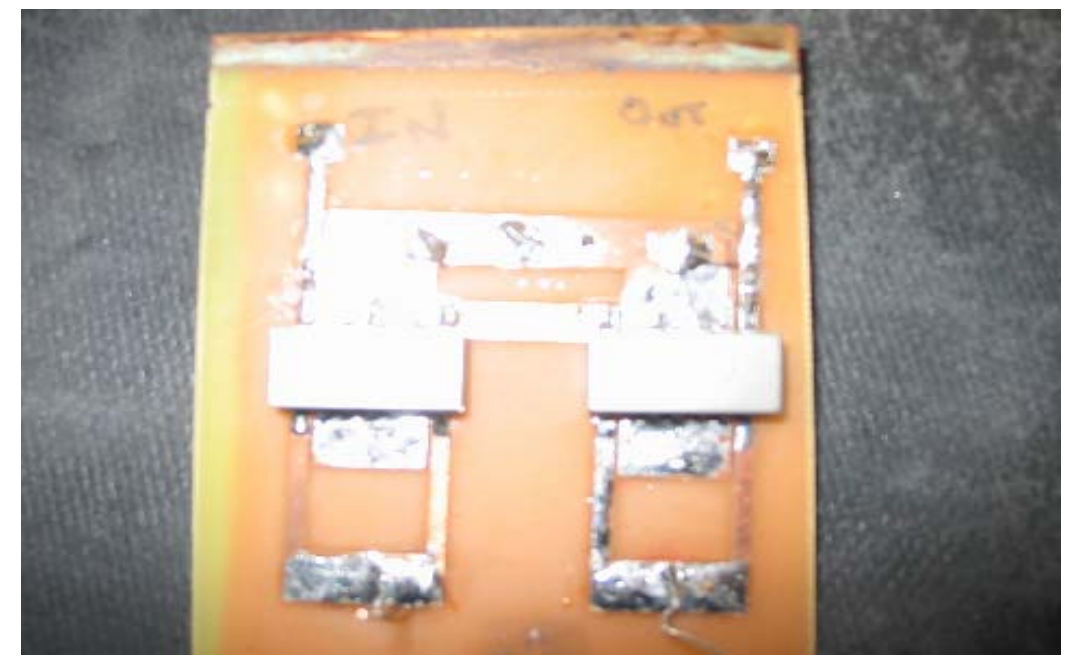

Figure 3.9. The Final PCB Design of the Phase Shifter

The PCB for the phase shifters were made in house using the PCB fabrication technique explained in Appendix A. The input and output of the board had SMA connectors. The AutoCAD layout of the board in shown in Figure 3.8, while the completed board is shown in Figure 3.9. The phase shifters were chosen to have no more than $1 \mathrm{~dB}$ of loss in the required bandwidth. 


\subsubsection{Coupler}

A MiniCircuits ZX30-9-4 coupler was used to sample the signal from an oscillator loop with a minimum drain of power. Coupler is a three port device having an input, output and coupled port. The output port with a $4 \mathrm{~dB}$ loss was connected to input of the amplifier. The coupled port having a loss of $9 \mathrm{~dB}$ with respect to the input was connected to the frequency counter. The coupler was chosen so that the minimum loss in the coupler at the direct port (from input port to output port). By placing the coupling network after the SAW and filters, much of the harmonic content from the saturated amplifier will be suppressed. The complete oscillator circuit is shown in Figure 3.10.

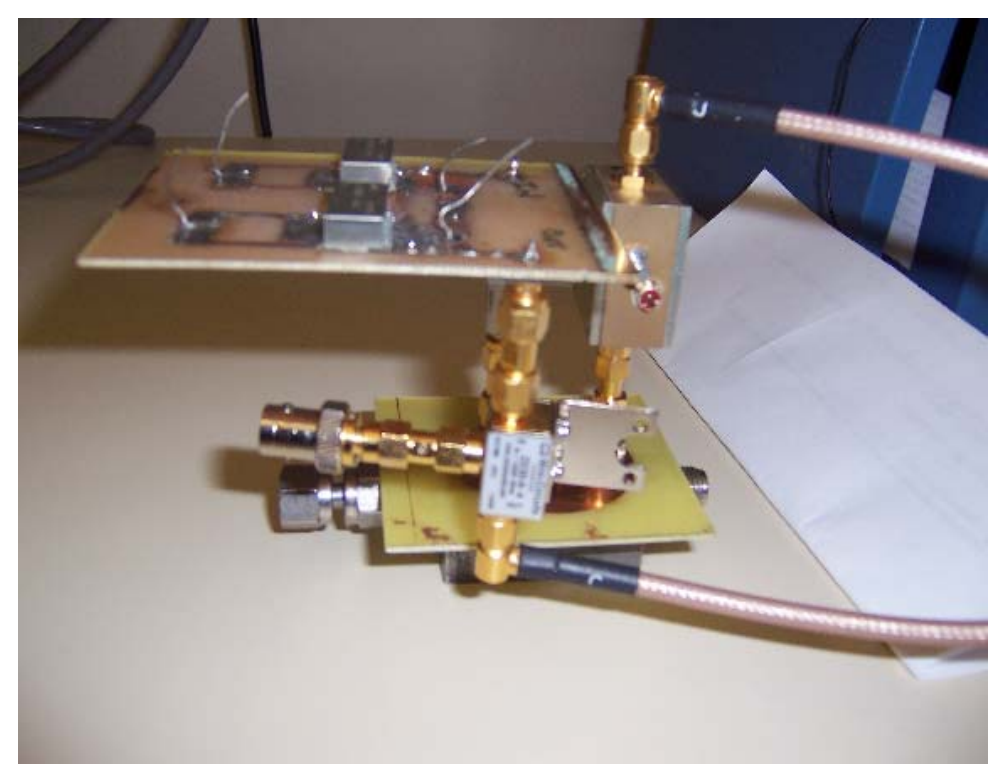

Figure 3.10. The Oscillator Circuit

\subsection{Automation}

A fully automated in house developed instrument control and data acquisition program with little modifications was used to accurately log sensor parameters and to reliably generate gas from the Mass Flow Controllers. The Data acquisition program was written using a LABVIEW (version 7) program. The program consisted of two main parts: 
1. Logging the sensor response

2. Generating the desired concentration of gas

Figure $3.11,3.12$ and 3.13 show the labview program used for data logging and instrument control. All experimental values were logged to a text file or excel sheet for processing and analysis.

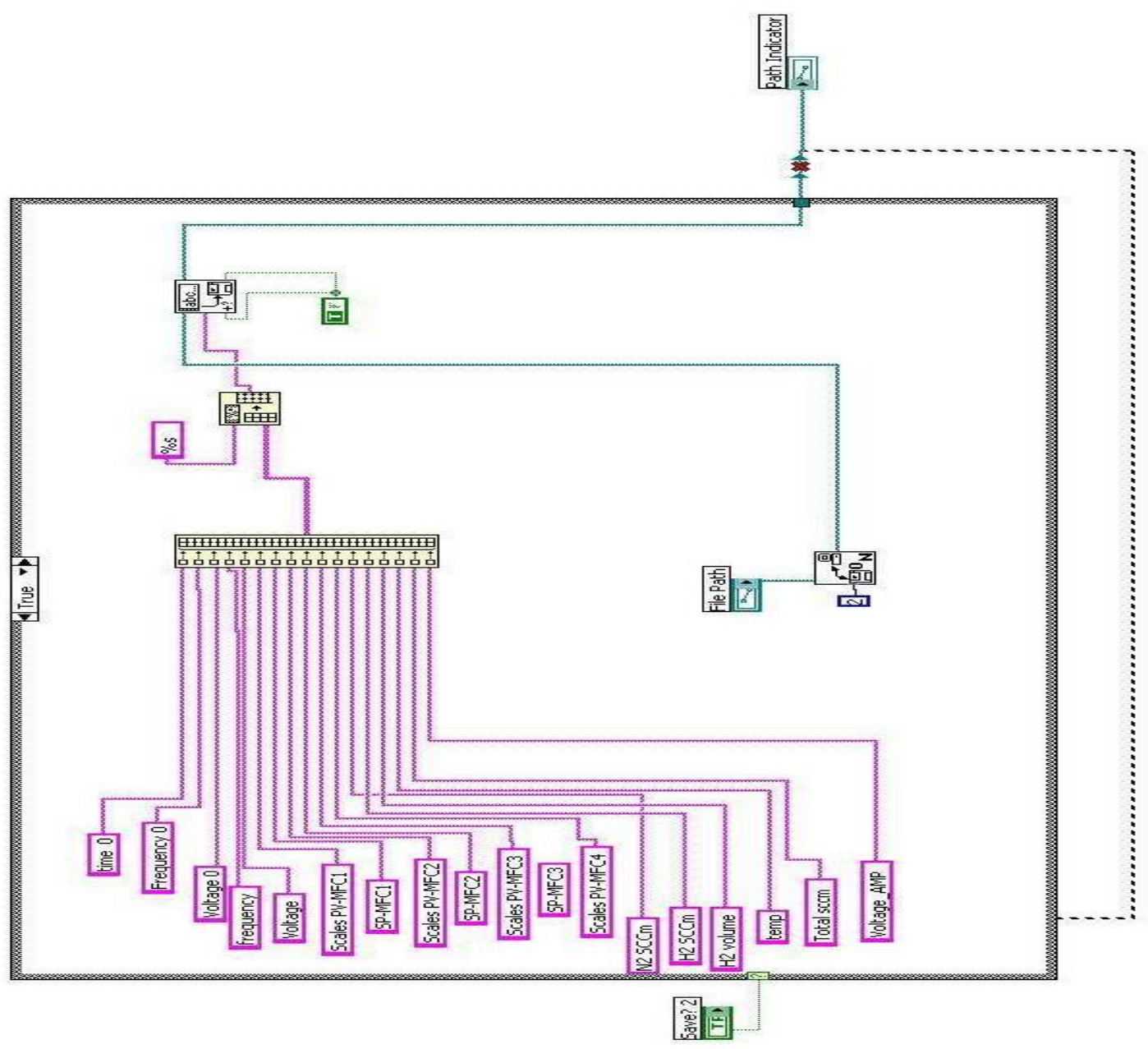

Figure 3.11. The LABVIEW Program for Writing Data Automatically into a Text File 


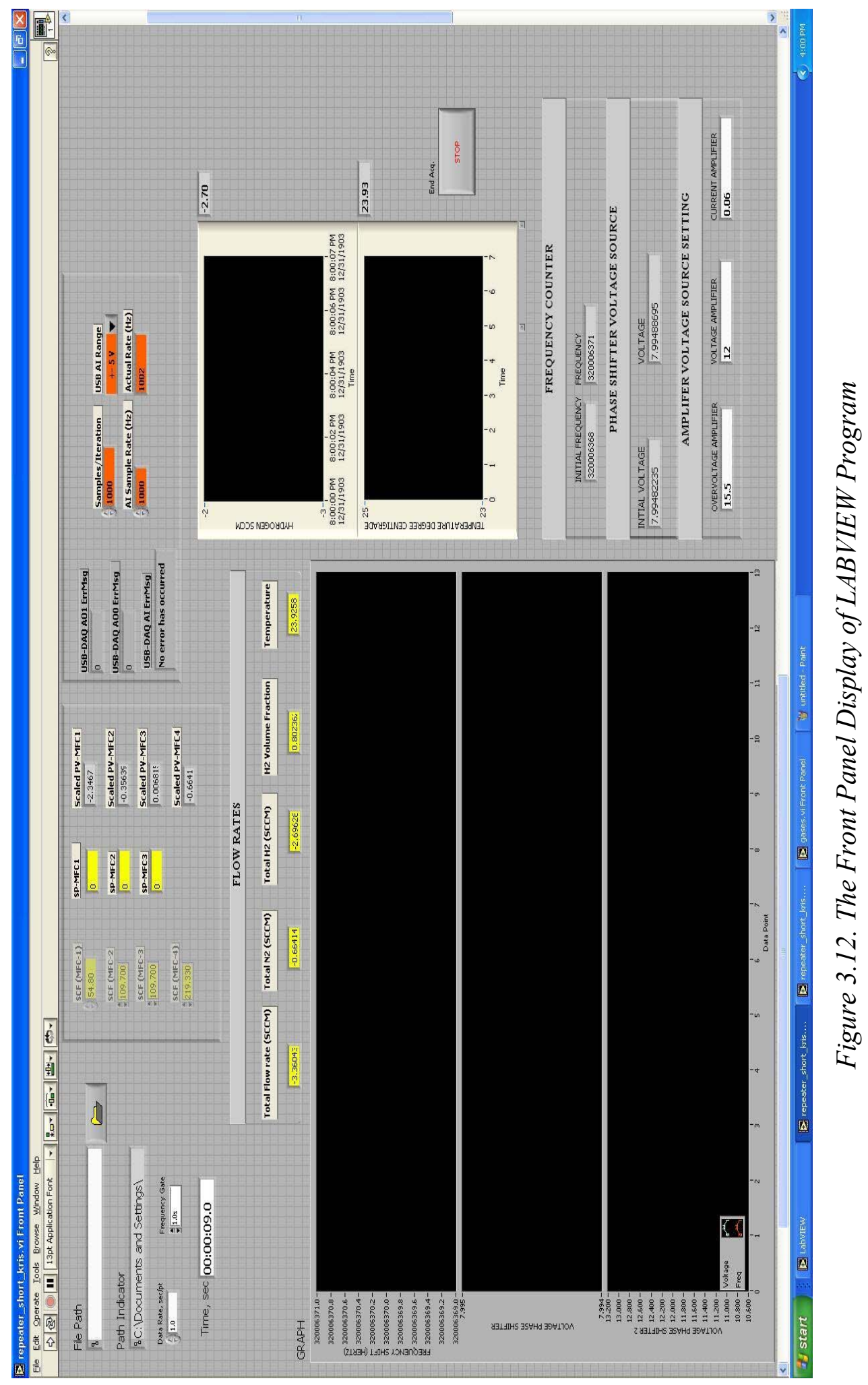




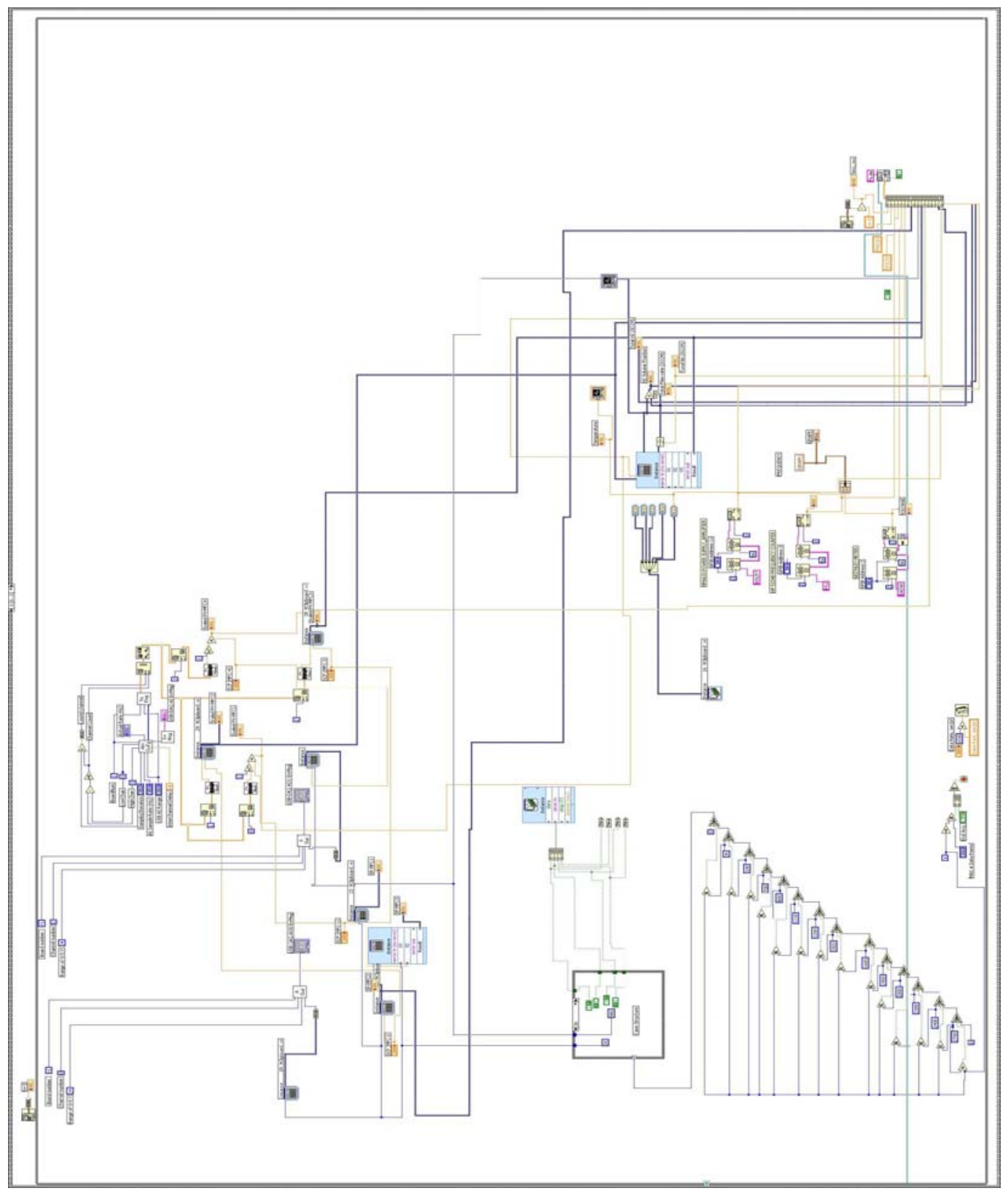

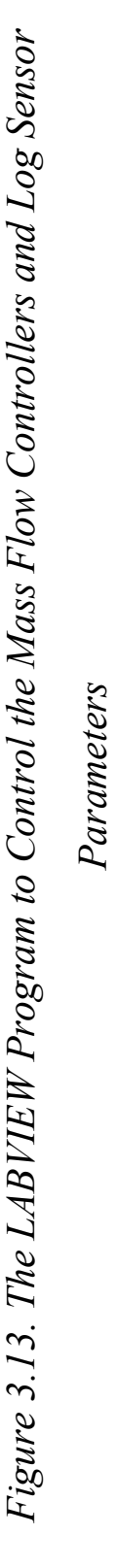




\section{CHAPTER 4: RESULTS}

\subsection{Tobacco Mosaic Virus}

An RF Monolithics, RP1239, $315 \mathrm{MHz}$, TO-39 package two-port SAW resonator was uncapped and used for the experiment. The $S_{21}$ characteristics of the uncapped resonator as measured with Agilent 8753ES S-Parameter Network analyzer is shown in Figure 4.1. The Figure shows the resonant frequency and corresponding attenuation. The SAW resonant frequency is $315.010750 \mathrm{MHz}$ with attenuation of $-3.5755 \mathrm{~dB}$ at the resonant frequency.

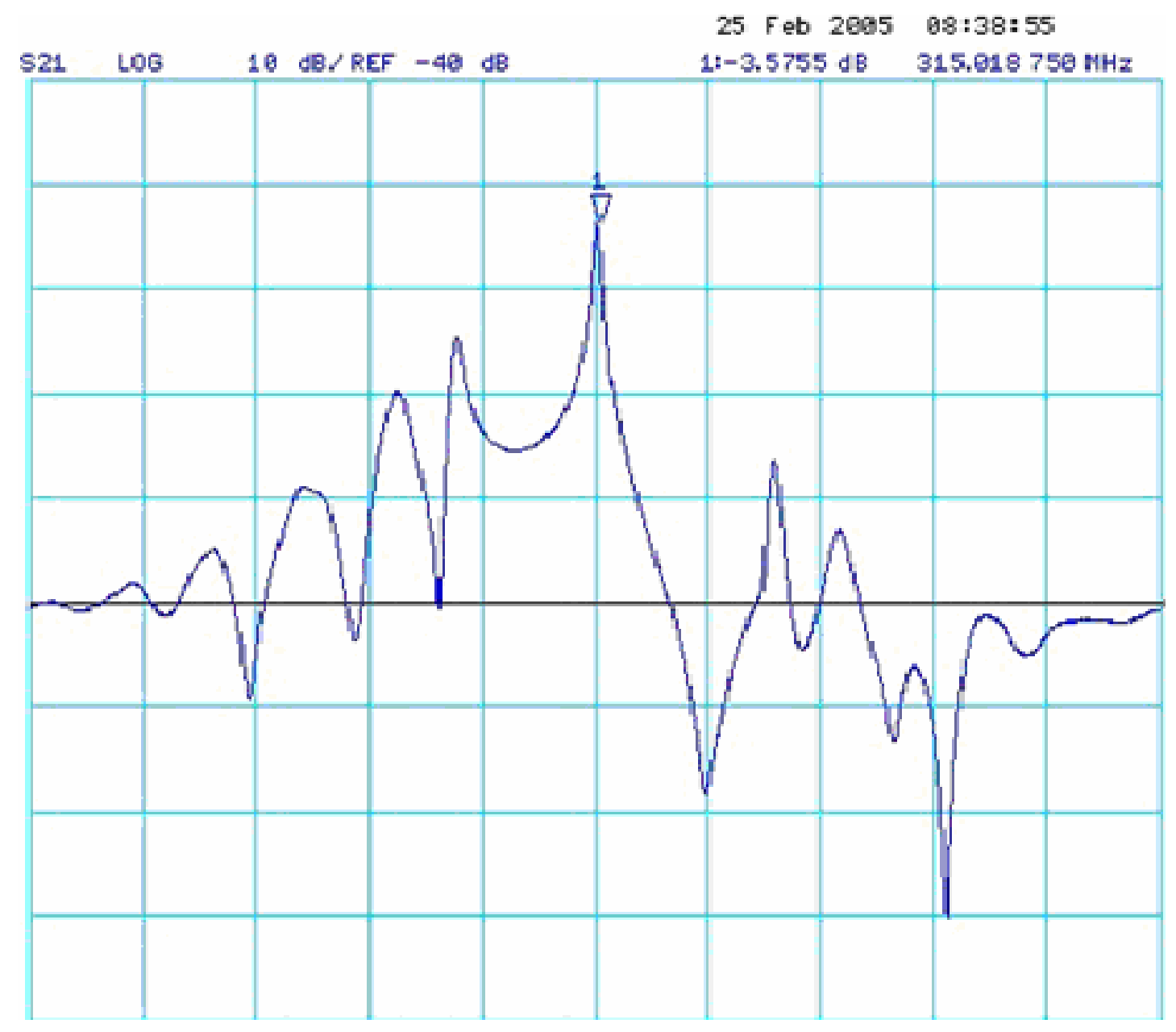

Figure 4.1. The Two-Port Characteristics of SAW RP1239 Two-Port Resonator 
The uncapped SAW resonator was then sputter coated with $1000 \AA$ of $\mathrm{SiO}_{2}$. The $\mathrm{Si}_{2}$ was used as an insulating layer to prevent the metallic nanoparticles from shorting the IDTs. The choice of $\mathrm{SiO}_{2}$ was justified in its ability to act both as an insulating and guiding layer thus promoting the propagation of waves through the medium $[31,32,33,34]$. The $\mathrm{SiO}_{2}$ coating caused an attenuation of the signal and a corresponding decrease in the resonant frequency of the device. Figure 4.2 shows the change in $\mathrm{S}_{21}$ characteristics of the device on $\mathrm{SiO}_{2}$ coating. The $\mathrm{SiO}_{2}$ coated SAW device was later drop coated with $20 \mu 1$ of 0.015 $\mathrm{mg} / \mathrm{mL} \mathrm{Pd}$ coated TMV. The Pd coated TMV samples were dispersed in 4DIMETHYLAMINOAZOBENZENE DIMETHYLAMINE-BORANE (DMAB) solution.

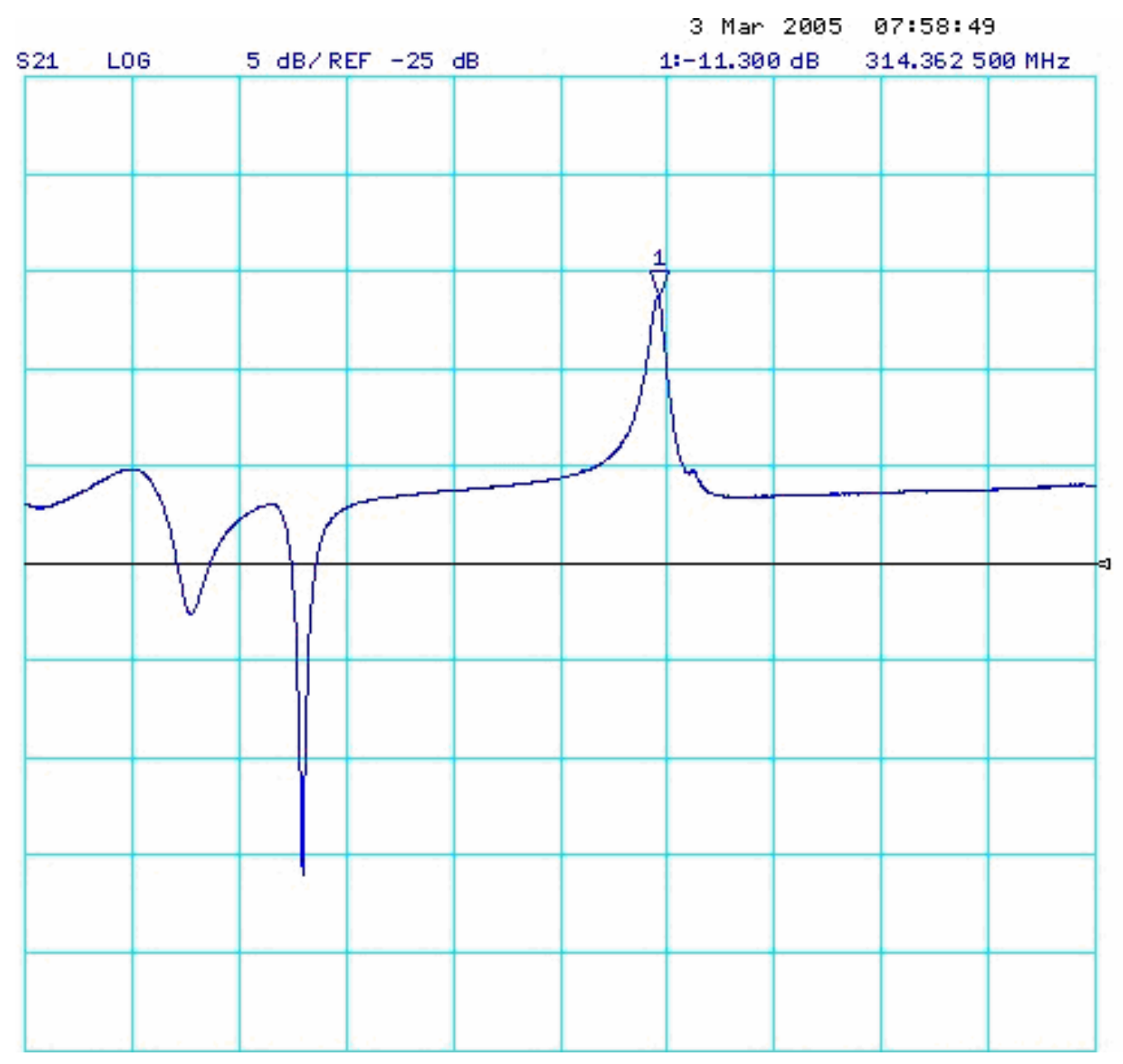

Figure 4.2. The Two-Port Characteristics of SAW RP1239 Two-Port Resonator after $\mathrm{SiO}_{2}$ Coating 


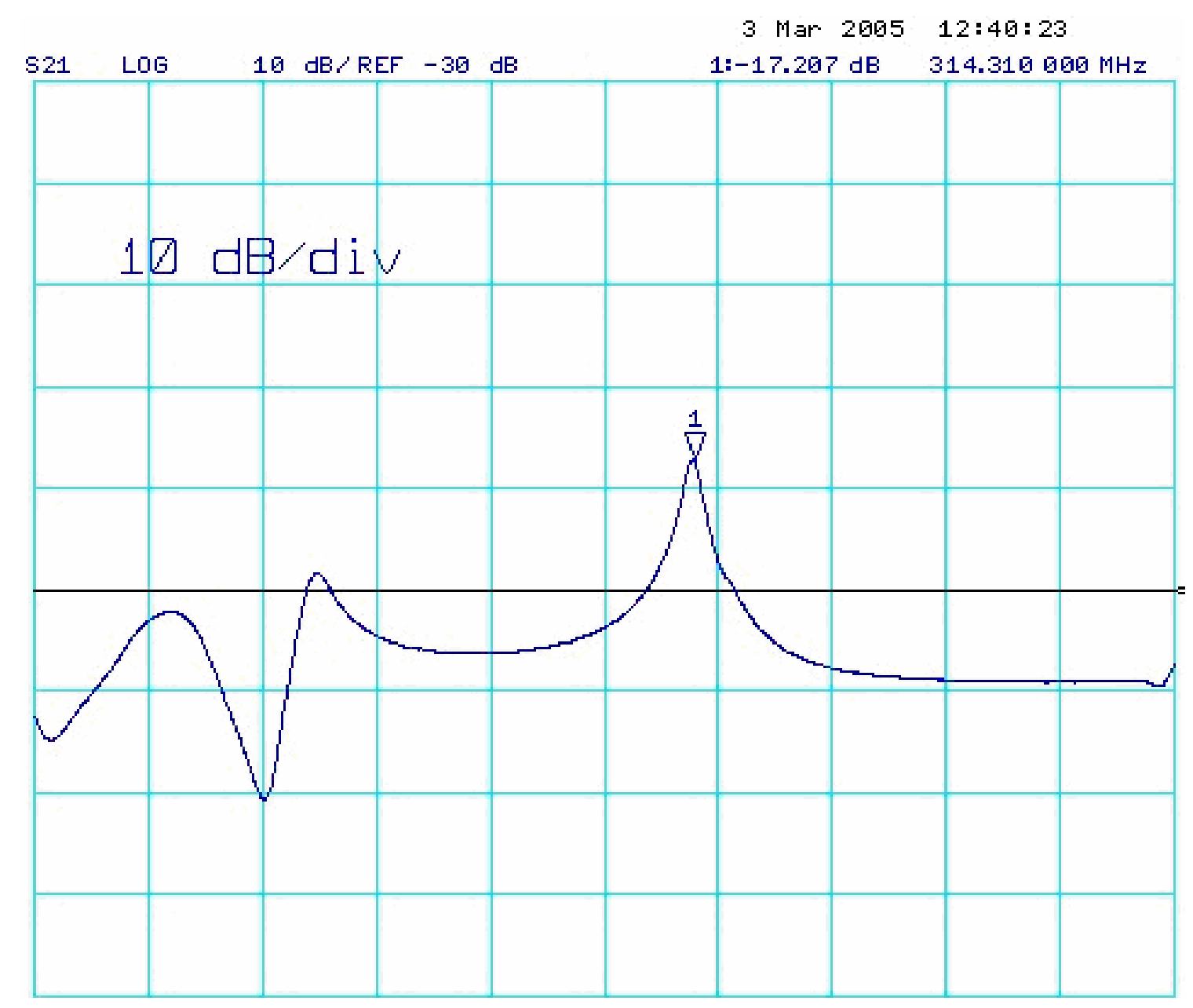

Figure 4.3. The Two-Port Characteristics of SiO ${ }_{2}$ Coated SAW RP1239 Two-Port

Resonators after TMV-Pd Coating

The coating of the sensing layer or Pd coated TMV particles produced a further attenuation of signal and decrease in resonant frequency of the device as seen from the $\mathrm{S}_{21}$ characteristics of the device in Figure 4.3. These attenuation and frequency changes have been tabulated in Table 4.1. The coated device was then soldered on the PCB and placed in the temperature controlled stainless steel cell. The seal was ensured to be leak proof. The resonator was also connected to the oscillator circuit to ensure that the fractional changes in velocity occurring due to different perturbation mechanisms discussed earlier can be tracked by changes in frequency. The coating of the sensing layer or Pd coated TMV particles produced a further attenuation of signal and decrease in resonant frequency of the device as seen from the $S_{21}$ characteristics of the device in 
Figure 4.3. These attenuation and frequency changes have been tabulated in Table 4.1. The coated device was then soldered on the PCB and placed in the temperature controlled stainless steel cell. The seal was ensured to be leak proof. The resonator was also connected to the oscillator circuit to ensure that the fractional changes in velocity occurring due to different perturbation mechanisms discussed earlier can be tracked by changes in frequency.

Table 4.1. SAW Resonator Frequency and Attenuation Measurements after Pd-TMV Coating

\begin{tabular}{|l|l|l|}
\hline Process & Frequency change & Attenuation change \\
\hline $1000 \AA ̊$ of SiO2. & $0.65625 \mathrm{MHz}$ & $-7.7245 \mathrm{~dB}$ \\
\hline $20 \mu \mathrm{l}$ Pd-TMV coating & $0.05250 \mathrm{MHz}$ & $-5 . .907 \mathrm{~dB}$ \\
\hline
\end{tabular}

The SAW device with the nanoparticle sensing layer was exposed to varying concentration of hydrogen. The varying concentrations of hydrogen were achieved by mixing hydrogen with nitrogen using MFCs and a mixing chamber, with the flow rate fixed at $1000 \mathrm{sccm}$. The flow rate was fixed to prevent seeing any flow related effects [6]. The entire process was automated using a LABVIEW program. The behavior of sensing film of exposure to $0.2-2.5 \%$ hydrogen is seen from Figure 4.4. The figure shows responses of the sensing film to varying volume $\%$ hydrogen. It is seen that the resonant frequency of the device increases on exposure to hydrogen. This behavior is contrary to mass loading wherein the absorption /adsorption of analyte results in decrease in frequency. This type of behavior is related to acoscuto-electric phenomenon wherein a layer of bound charges developed in the piezoelectric material by passing SAW is redistributed by the presence of a conducting layer. This redistribution causes compensation of bound charges developed due to the passing surface wave [16]. This phenomenon usually results in producing an increase in frequency. The corresponding response times were calculated to be around $30 \mathrm{sec}$. It was observed that the process of absorption/desorption of hydrogen from the sensing film was reversible. 

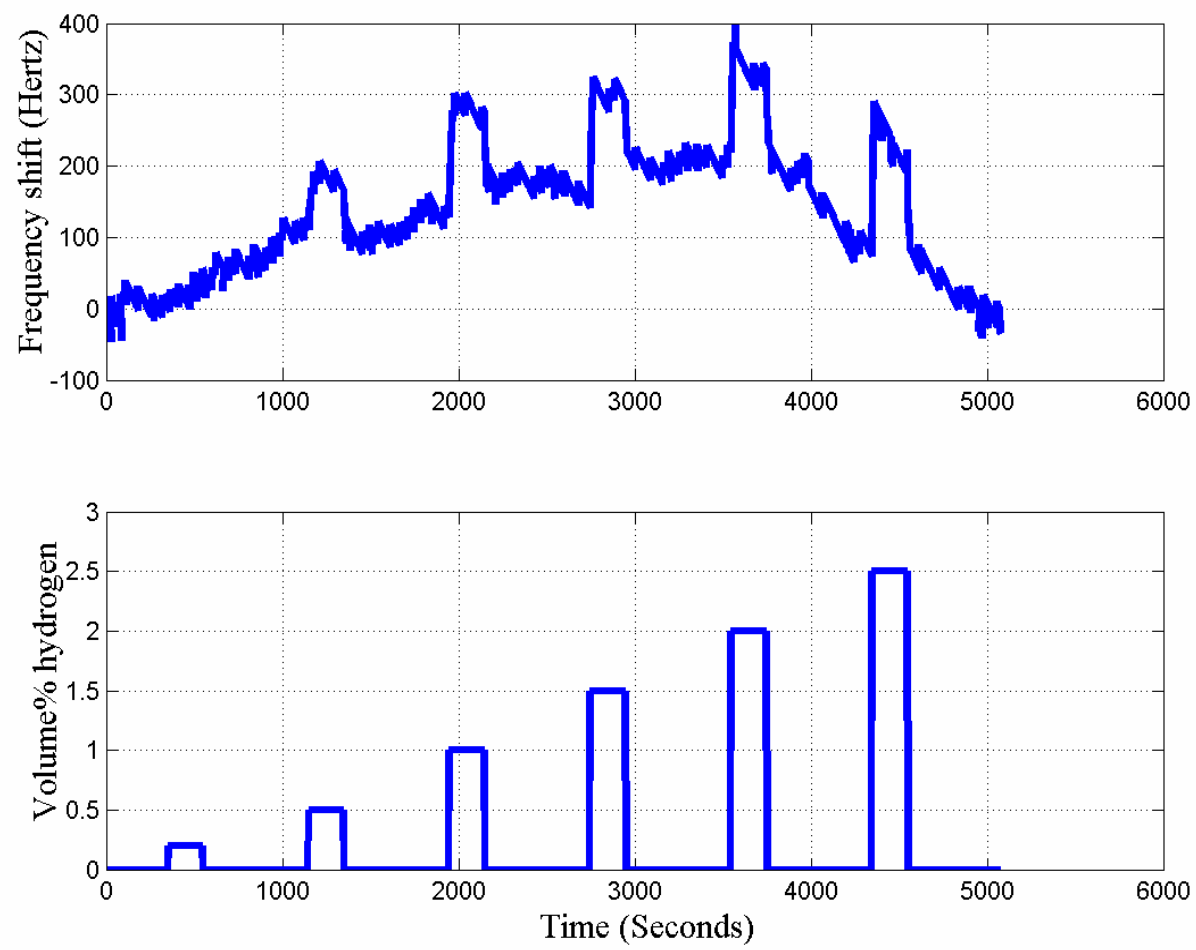

Figure 4.4. The Response of Pd Coated TMV to 0.2-2.5\% Hydrogen

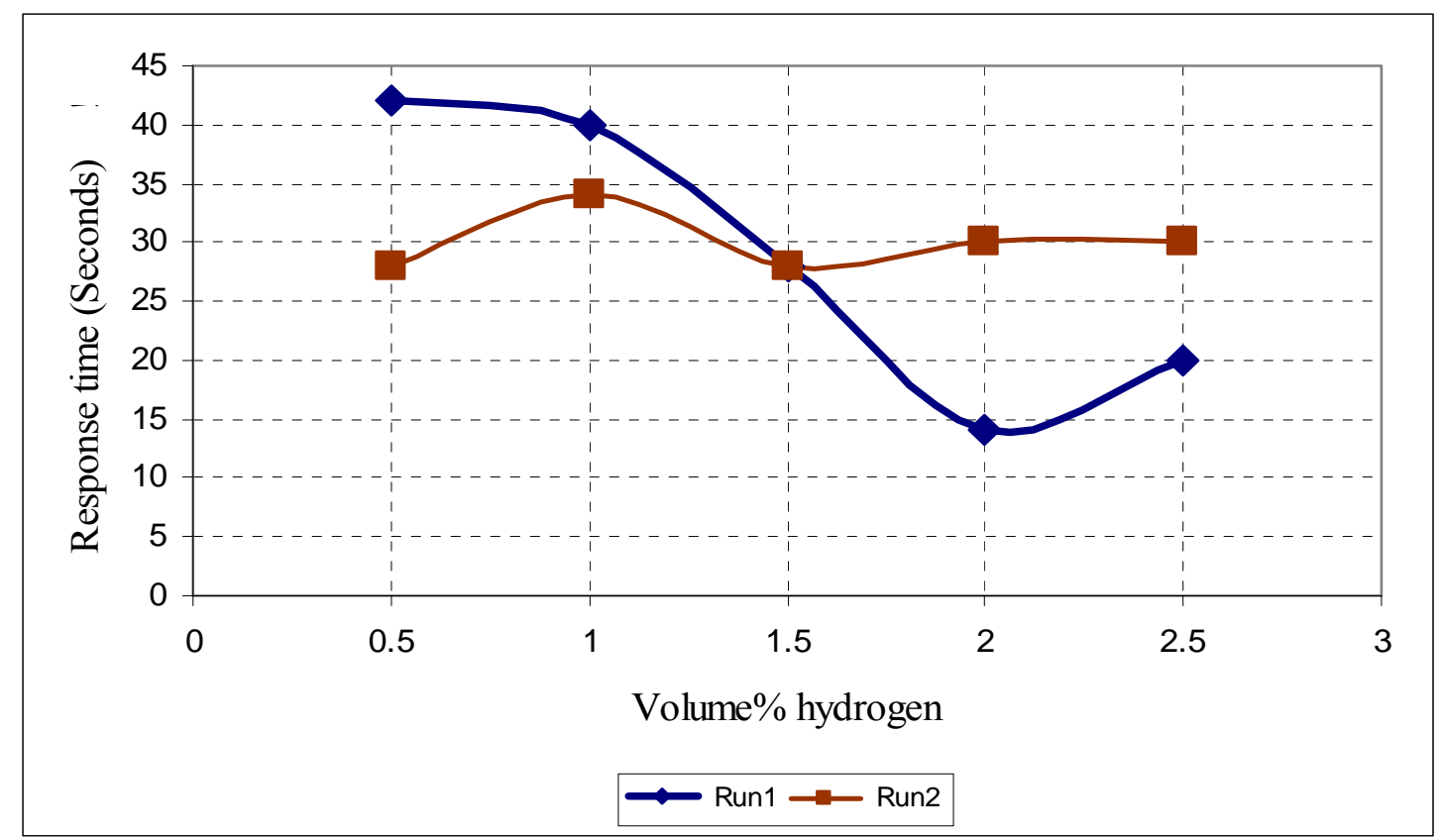

Figure 4.5. The Response Times for Pd Coated TMV Resonator in Response to 0.2-2.5\% Hydrogen 
The response times for $99 \%$ of full response for two different runs of $0.2-2.5 \%$ hydrogen are shown in Figure 4.5. It is seen from the graph that the response times are have an average value of 30 seconds. The values at $0.2 \%$ were very small to be discernable, due to which their values have not been graphed. The calibration curve is shown in Figure 4.6 which shows a concentration independent response. The sensing film shows good response times for $99 \%$ of full response.

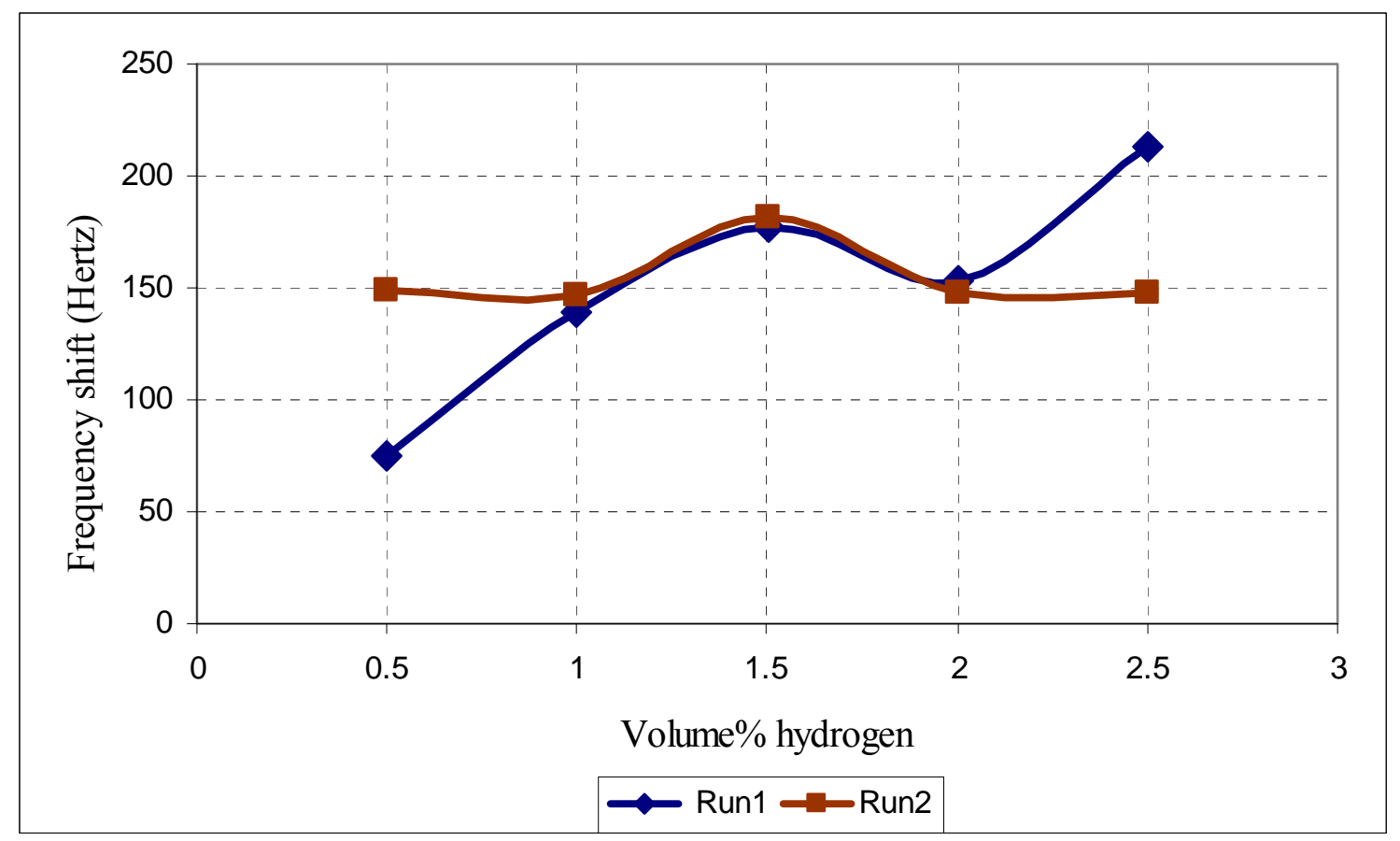

Figure 4.6. The Frequency Shifts Produced for Two Different Runs at Various Hydrogen Concentrations

The sensing film was also tested to $2.5-4 \%$ hydrogen in nitrogen. These tests were carried out to test below the lower explosion limits of hydrogen ( $4.65 \%$ in air). The response of the sensing layer on hydrogen exposure has been plotted in Figure 4.7. The responses reaffirm the fact that the analyte absorption causes the frequency of the device to increase. It is also seen from the responses that the process absorption/desorption of hydrogen from the sensing film is reversible. The response times and frequency shifts produced by $2.5-4.5 \%$ hydrogen concentrations have also been plotted in Figure 4.8 and Figure 4.9. 

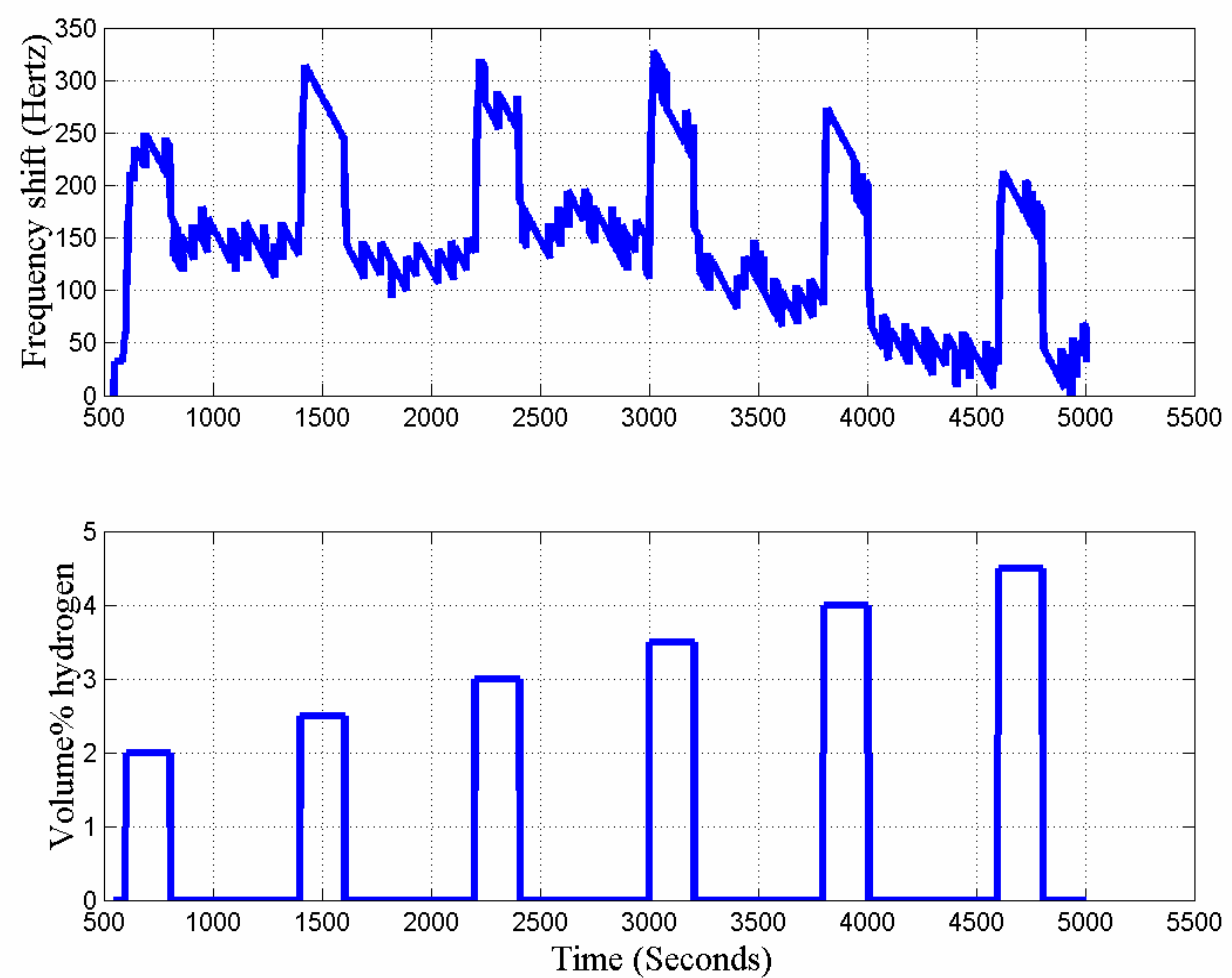

Figure 4.7. The Sensing Film Response to 2-4.5\% Hydrogen

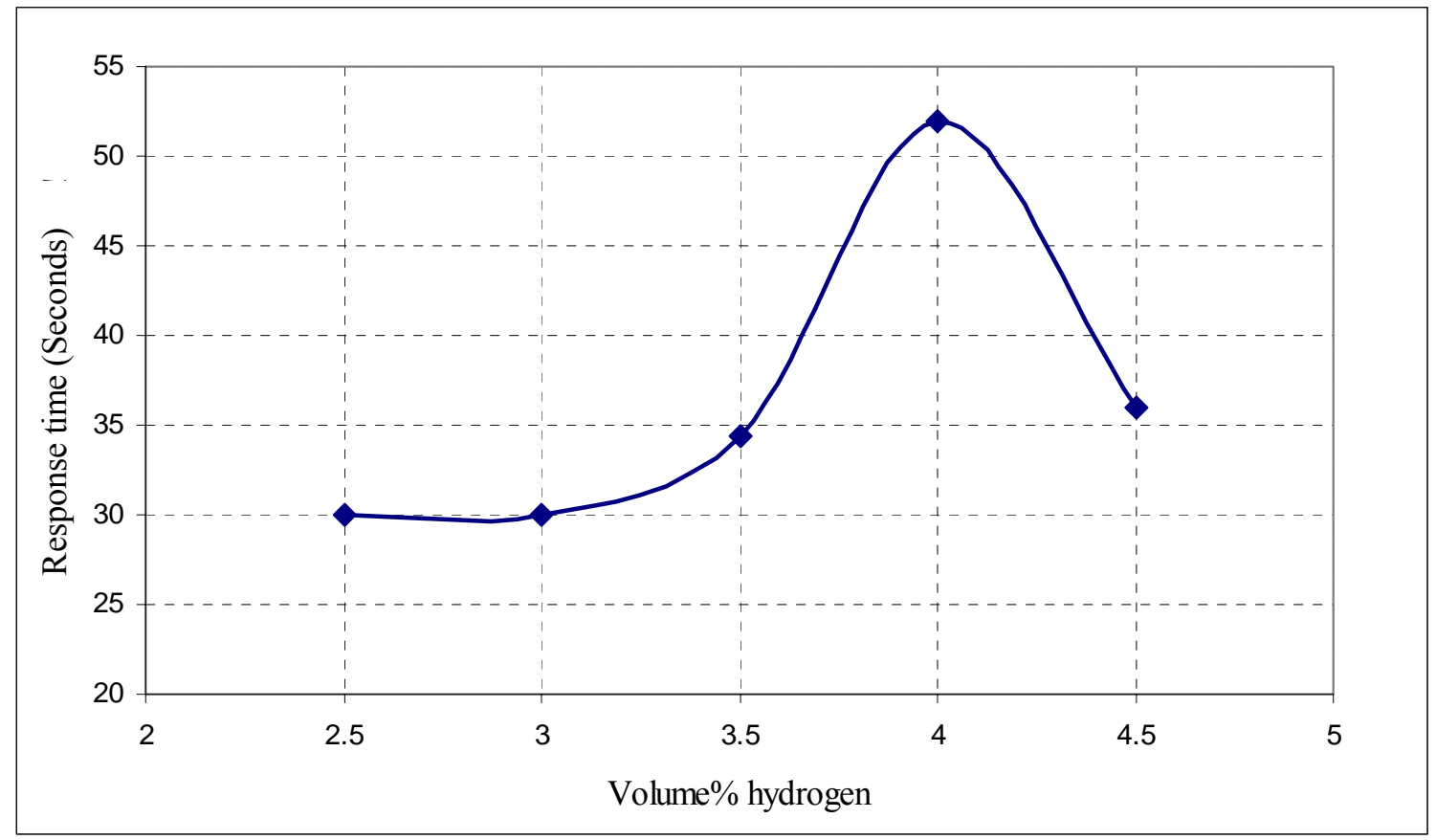

Figure 4.8. The Response Times of Sensing Film for 2-4.5\% Hydrogen 


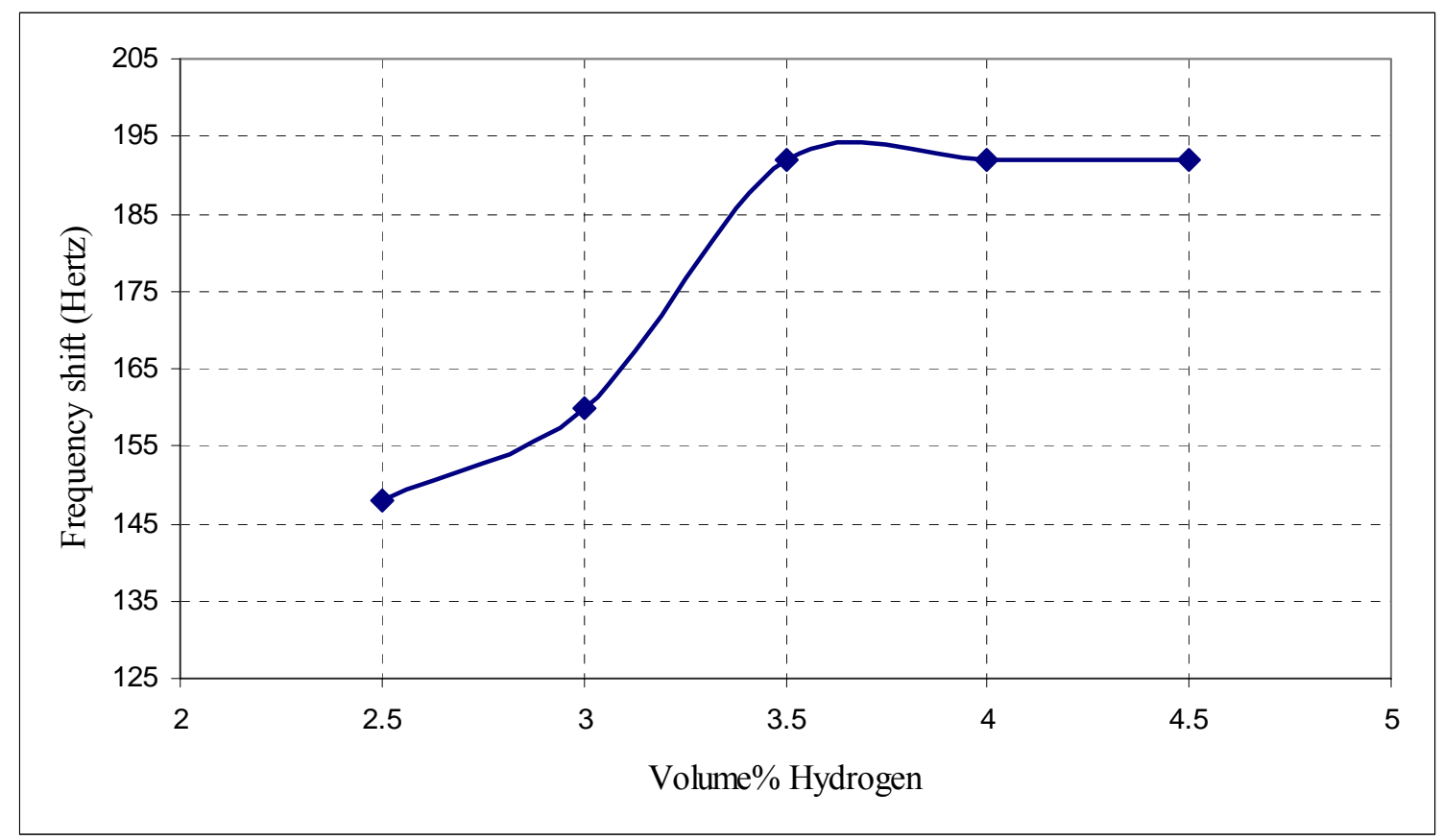

Figure 4.9. The Frequency Shift Produced in the Sensing Film 2.5-4.5\% Hydrogen

From the response curves shown previously it can be seen that the responses were repeatable over several runs. It was also observed that the process of absorption and desorption of hydrogen in the sensing film was reversible. Previous work by our group on nano-structured thin film Pd SAW sensors has shown that repeated absorption and desorption of hydrogen in the sensing film causes the palladium film to crack and peel [30], which was not seen to be the case with palladium nanoparticle-coated Tobacco Mosaic Virus sensing layers. The cracked and peeled Pd film upon hydrogen sorption /desorption is shown in Figure 4.10 [30] and compared with the hydrogen exposed (repeatedly) Pd-TMV layer in Figure 4.11. Also, the Pd film based SAW sensors show effects of aging after few days of not being exposed to hydrogen [35]. The devices have to be exposed to hydrogen for some time before they start responding in the desired manner. The Pd-TMV coated devices were seen to respond without any need for activation cycling after weeks of testing. The sensor noise was calculated to be $17 \mathrm{~Hz}$ approximately which was defined as standard deviation of the mean frequency calculated over a 10 minute interval. Since the response frequency shift magnitudes are small, Pt 
coated TMV samples were also tested for their response to hydrogen. These devices showed no response to hydrogen challenges.

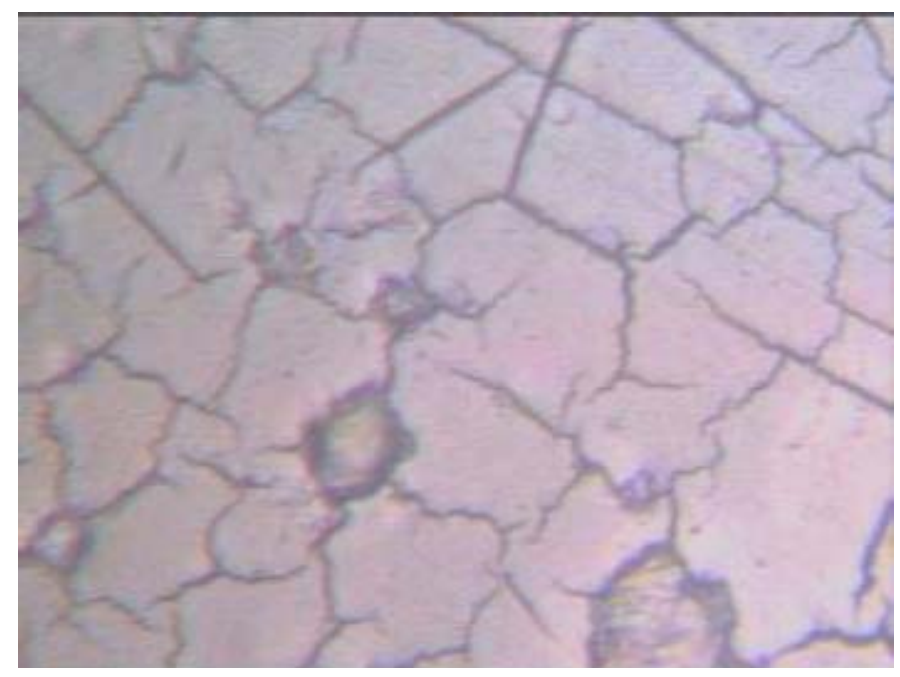

Figure 4.10. Cracking of Palladium Film Upon Hydrogen Sorption/Desorption

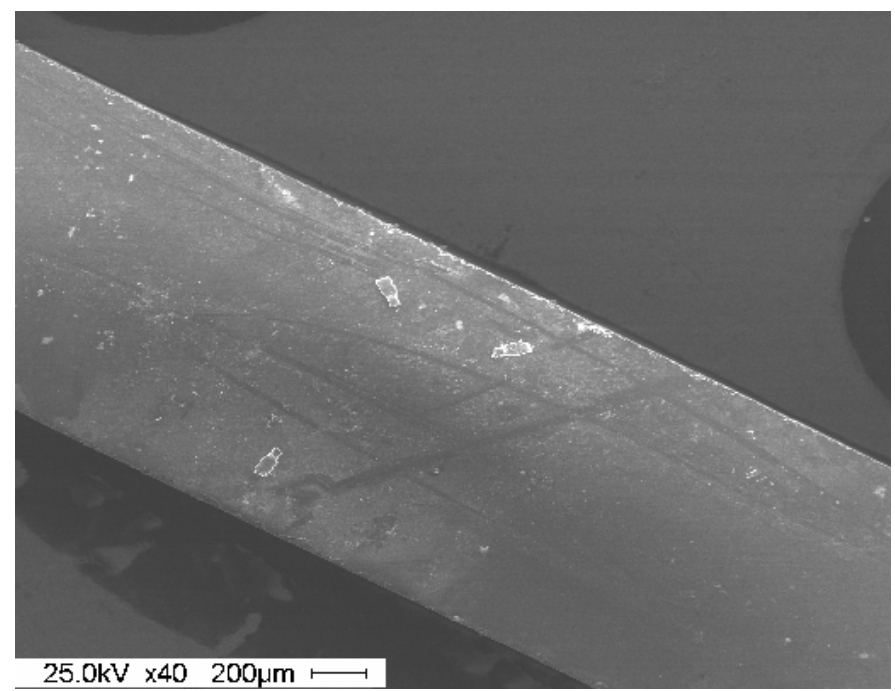

Figure 4.11. Device Surface Coated with Palladium Nanoparticle-Coated Tobacco Mosaic Virus Film Free of Rearrangement 


\subsection{Carbon Nanotubes}

One more RF monolithic RP 1239 SAW two-port resonator was uncapped and sputter coated with $1000 \AA$ of $\mathrm{Si}_{2}$. The $\mathrm{Si}_{2}$ coated resonator was then drop coated with $10 \mu \mathrm{L}$ sample of $0.167 \mathrm{mg} / \mathrm{mL}$ SWNTs dispersed in $1 \%$ SDS in $\mathrm{D}_{2} \mathrm{O}$ solution. This resonator was evaporated with $27 \AA$ of palladium using electron beam evaporation. To evaluate the changes in resonant frequency and attenuation due to addition of $\mathrm{SiO}_{2}$ and sensing layer the $S_{21}$ parameters were obtained from Agilent 8753ES S-parameter Network Analyzer. The SAW resonant frequency was found to be $315.010750 \mathrm{MHz}$ with attenuation of $-3.5755 \mathrm{~dB}$ at the resonant frequency. The changes due to various coating have been tabulated in Table 4.2.

Table 4.2. SAW Resonator Frequency and Attenuation Measurements after Pd-SWNT Coating

\begin{tabular}{|c|c|c|}
\hline Process & Frequency change & Attenuation change \\
\hline $1000 \AA$ of $\mathrm{SiO}_{2}$ & $0.64315 \mathrm{MHz}$ & $-7.200 \mathrm{~dB}$ \\
\hline $10 \mu \mathrm{SWNT}$ solution & $0.40000 \mathrm{MHz}$ & $-6.432 \mathrm{~dB}$ \\
\hline $27 \AA$ of Palladium & $0.10000 \mathrm{MHz}$ & $-2.138 \mathrm{~dB}$ \\
\hline
\end{tabular}

It was seen that the drop coating of SWNTs and evaporation of caused an attenuation of the signal and a corresponding decrease in the resonant frequency of the device. The resonator was fixed on a PCB and placed in the Stainless Steel cell to be in contact with the incoming gas. This resonator was also made a part of oscillator loop to track the fractional changes in frequency.

The resonator with the sensing film was exposed to $0.2-2.5 \%$ volume $\%$ hydrogen in nitrogen at a flow rate of $1000 \mathrm{sccm}$. The response of the sensing film has been plotted in Figure 4.12. In accordance with a mass-loading response mechanism, frequency decreases were observed upon hydrogen exposures [16]. These shifts are seen to be quite large, keeping in mind that only a $27 \AA$ layer of Pd was deposited on the SWNTs. Figure 
4.13 shows the response times, defined as time taken to reach nearly $99 \%$ of complete response, for $0.2-2.5 \%$ of hydrogen exposures.
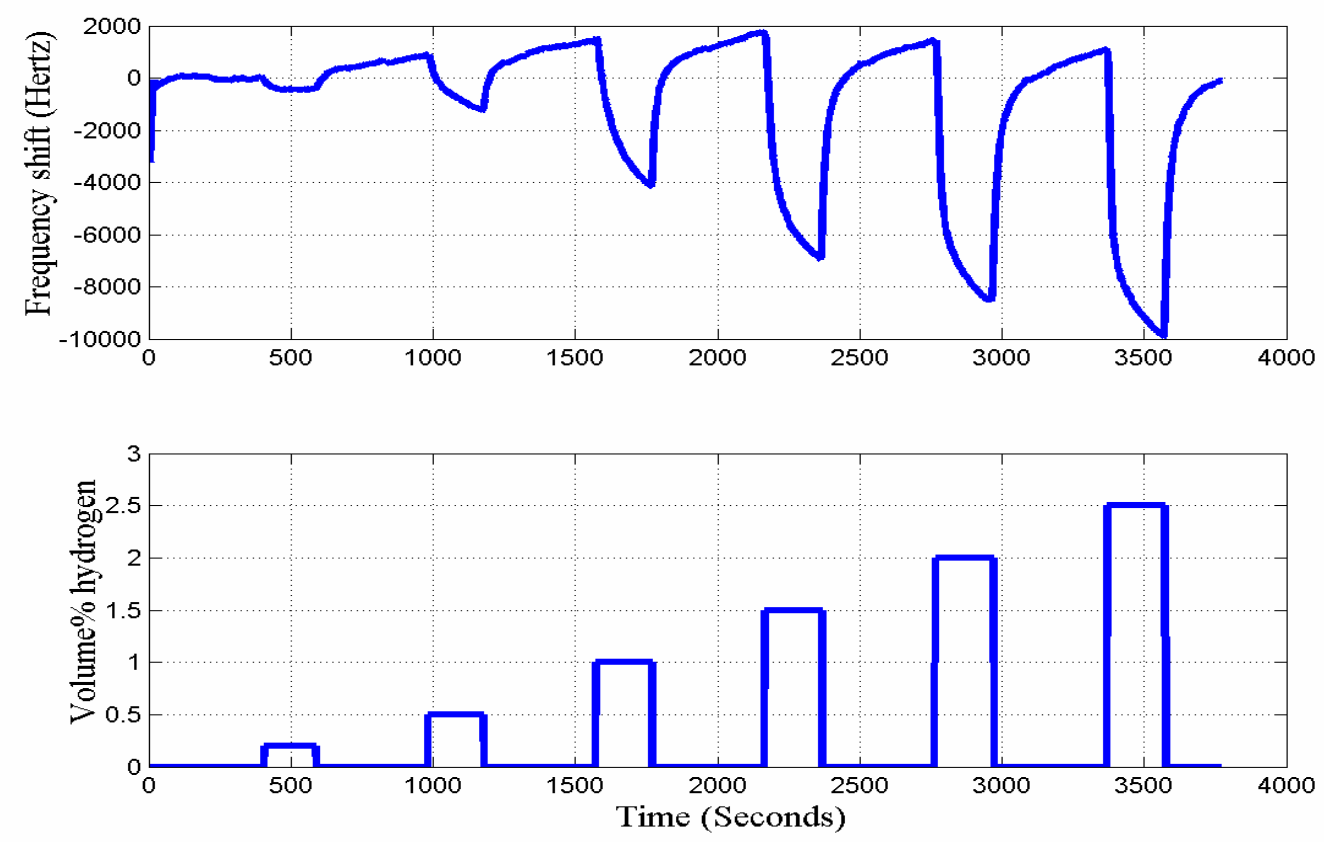

Figure 4.12. The Curve Showing the Response of Pd Coated SWNTs Response to Hydrogen

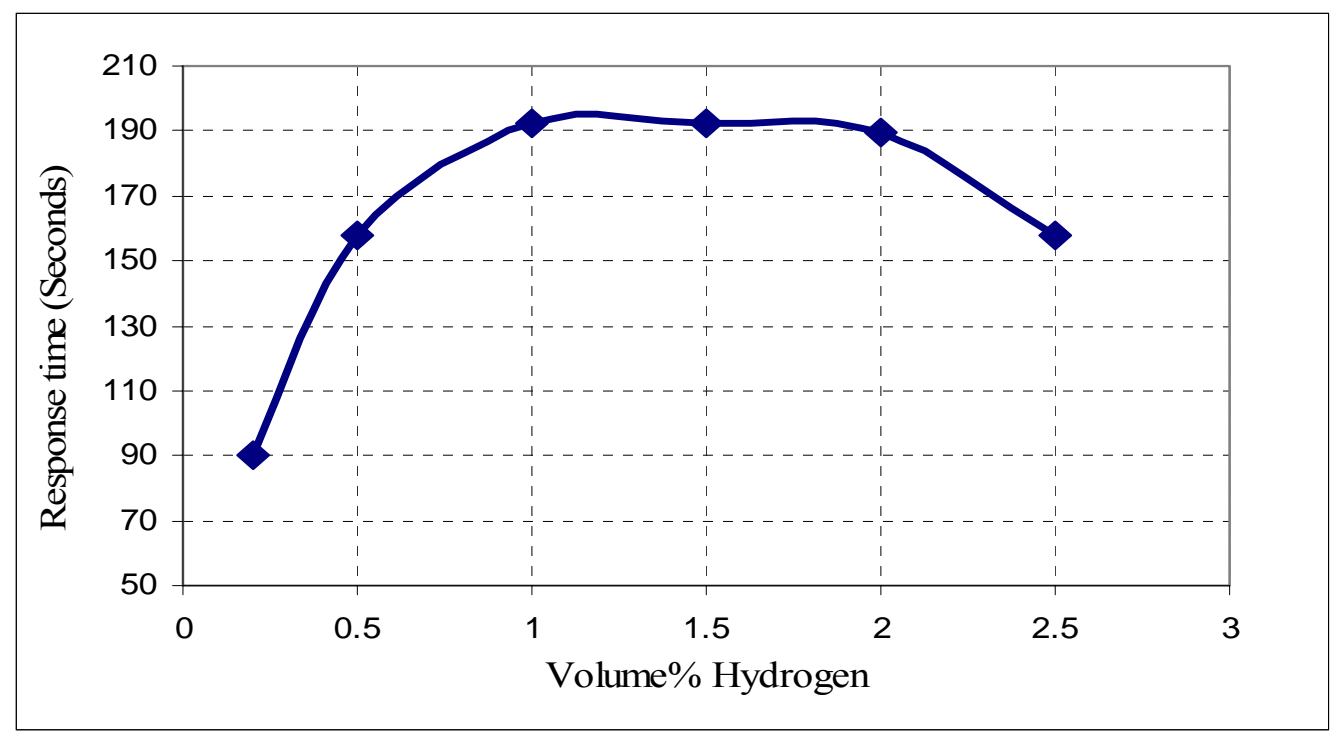

Figure 4.13. Response Times for Pd-SWNTs Coated SAW Resonator to 0.5-2.5\% Hydrogen Exposures 
These are seen to be about 175 seconds. While these are larger than for the PdTMV films, it should be noted that the large and immediate response reflected in the $66 \%$ response time (see Figure 4.15) allows for fast-responding hydrogen sensors using PdSWNTs to be constructed. The device calibration curve is shown in Figure 4.14, which clearly indicates a concentration dependant frequency shift, unlike for the Pd-TMV layer tested

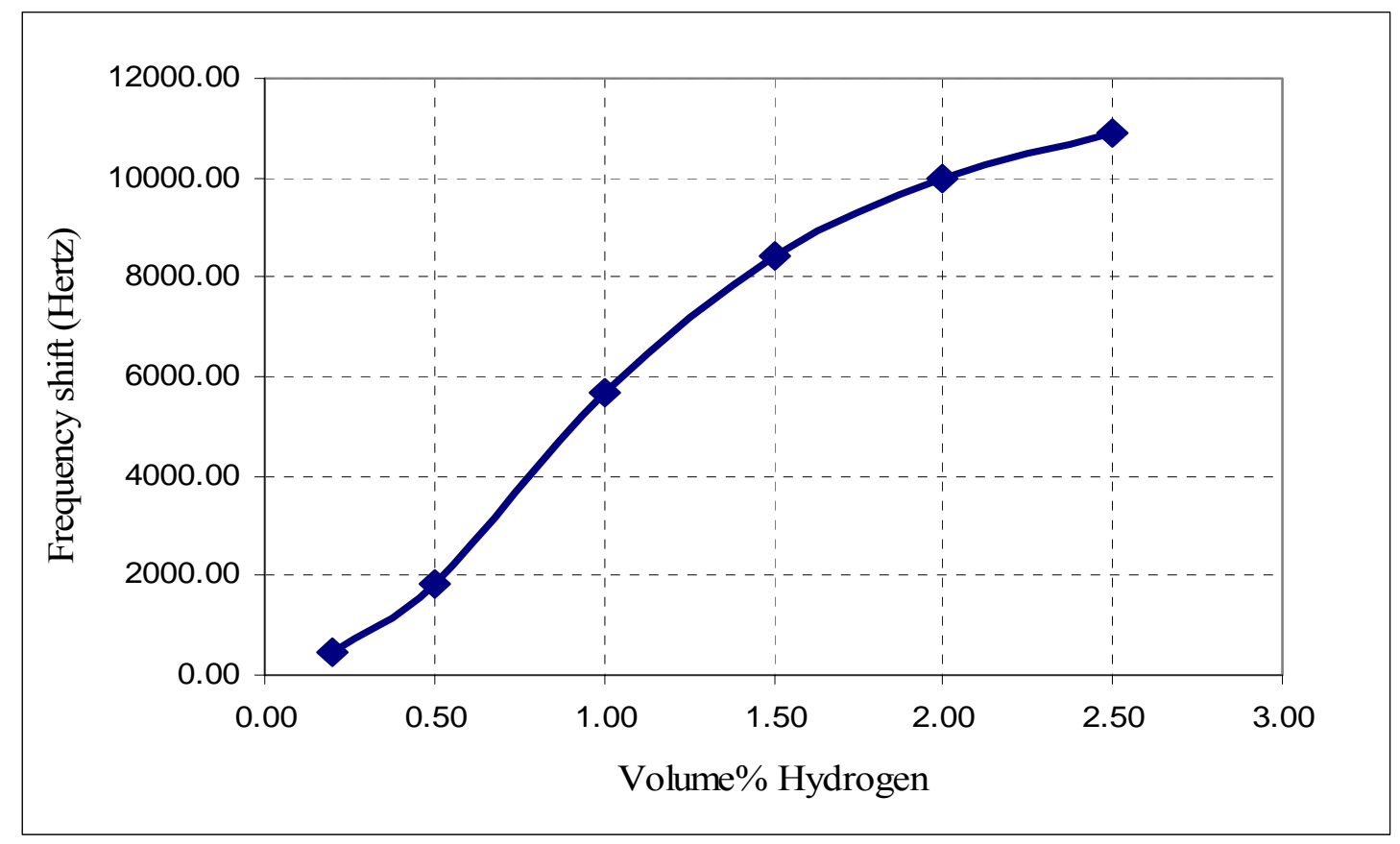

Figure 4.14. Frequency Shifts for Pd-SWNTs Coated SAW Resonator to 0.2-2.5\% Hydrogen Exposures

The Pd coated SWNT film was later exposed to several runs of fixed concentrations to see if there is any change in the film characteristics in terms of amount of shift produced and the response times. From Figure 4.15 and Figure 4.16 it is seen that repeated exposures to 1 and $1.5 \%$ produces no appreciable change in terms of amount of shift produced and the response times. The frequency shifts are seen to be around $6 \mathrm{KHz}$ and $8 \mathrm{KHz}$ at $1 \%$ and $1.5 \%$ hydrogen respectively. The comparison of these frequency shifts to the calibration curve (Figure 4.14) show no appreciable change in the amount of shifts. This shows that film was robust and did not suffer from problems of peeling, degradation and cracking from repeated exposures. 

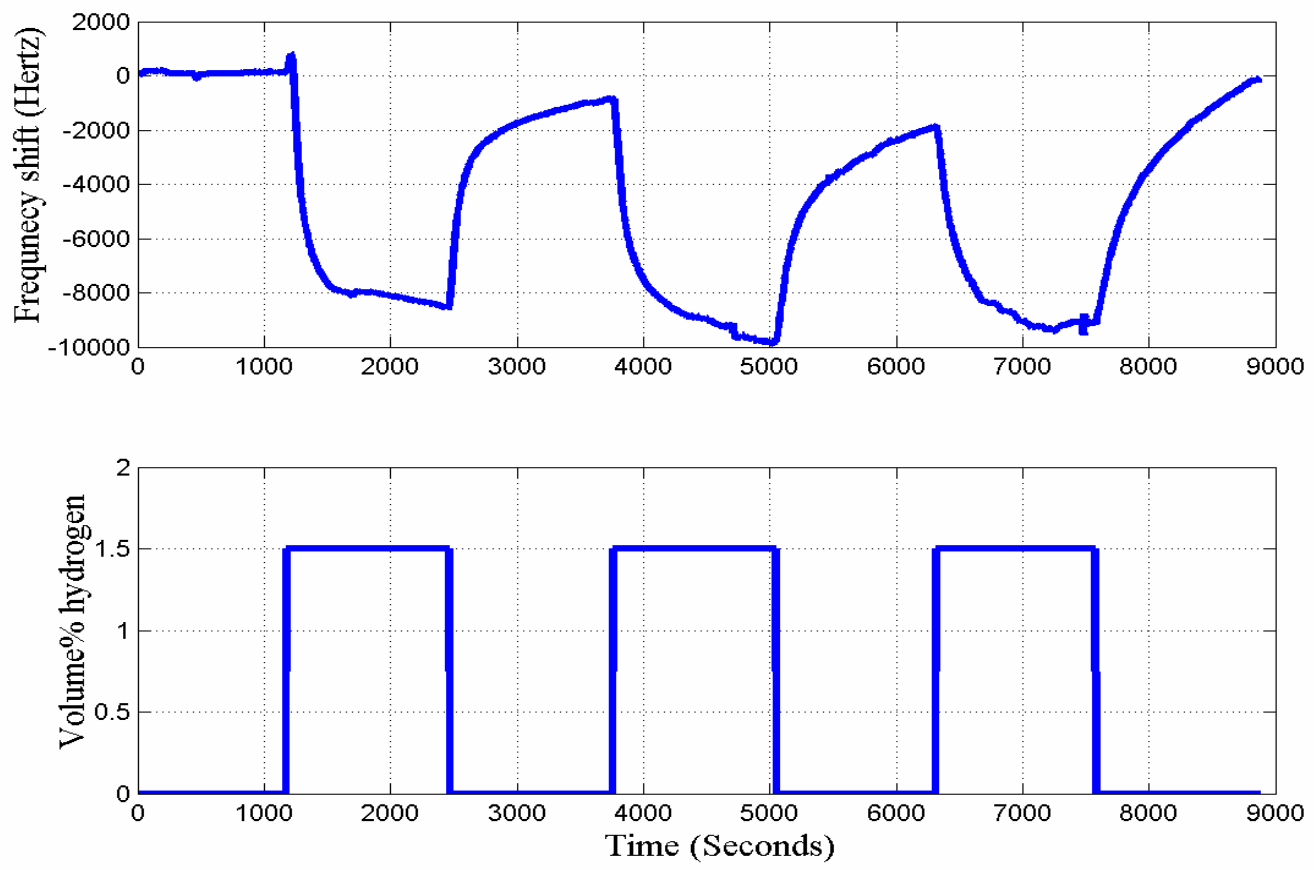

Figure 4.15. Frequency Shifts for Pd-SWNTs Coated SAW Resonator to $1.5 \%$ Hydrogen
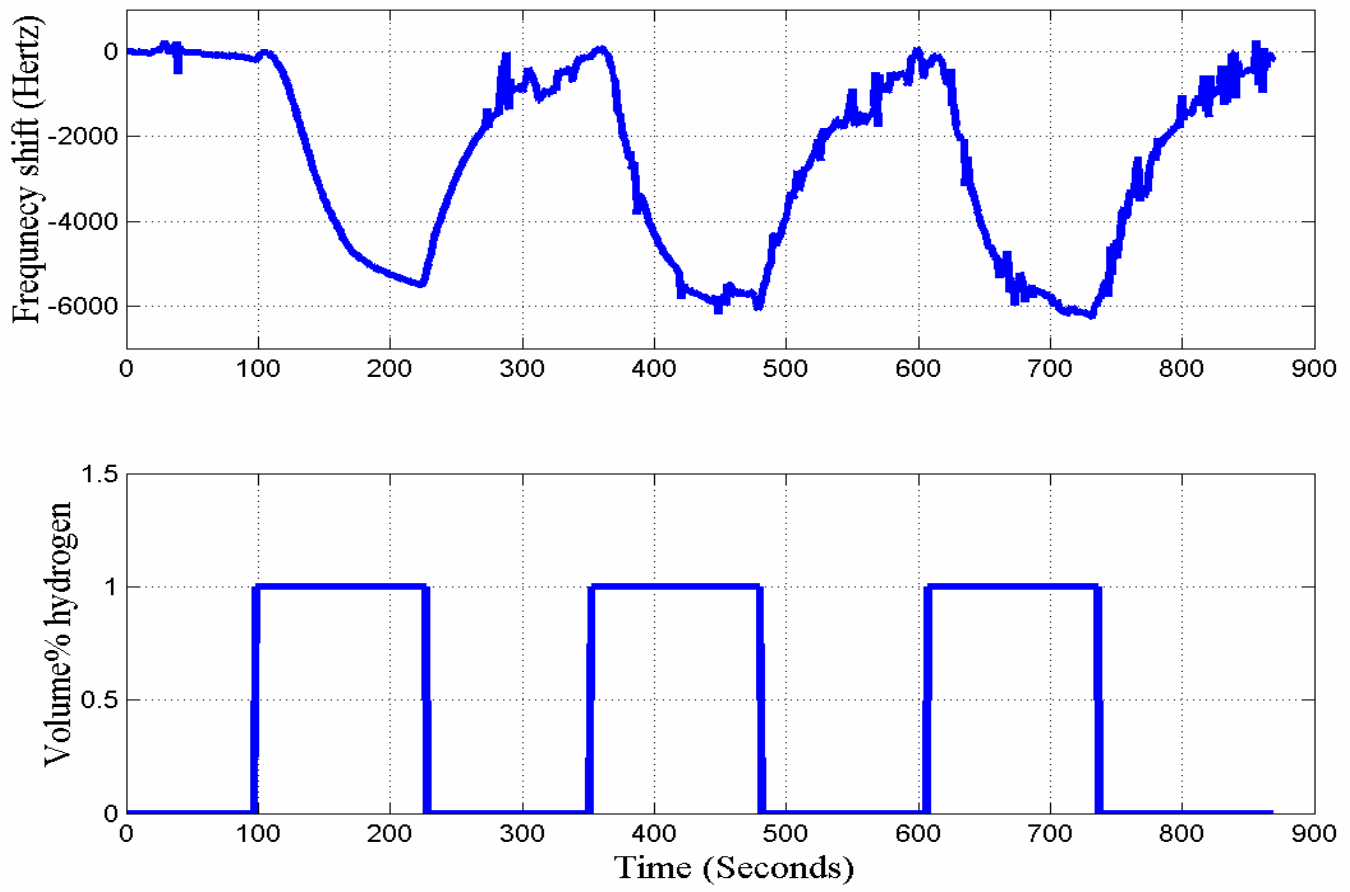

Figure 4.16. Frequency Shifts for Pd-SWNTs Coated SAW Resonator to $1 \%$ Hydrogen 
From the response curves shown previously it can be seen that the responses were repeatable over several runs. It was also observed that the process of absorption and desorption of hydrogen in the sensing film was reversible. The film was robust since repeated runs at varying concentrations and fixed concentrations didn't seem have produced any appreciable changes in terms of response times and corresponding frequency shifts. Also the film did not show any signs of peeling or degradation.

\subsection{Palladium Film}

One more RF monolithic RP 1239 SAW two-port resonator was uncapped and sputter coated with $1000 \AA$ of $\mathrm{SiO}_{2}$. The $\mathrm{SiO}_{2}$ coated resonator was coated with $27 \AA$ of palladium by e-beam evaporation process. To evaluate the changes in resonant frequency and attenuation due to addition of $\mathrm{SiO}_{2}$ and $\mathrm{Pd}$ sensing layer the $\mathrm{S}_{21}$ parameters were obtained from Agilent 8753ES S-parameter Network Analyzer. The changes due to various coating have been tabulated in Table 4.3. The uncoated SAW resonant frequency was found to be $315.010750 \mathrm{MHz}$ with attenuation of $-3.5755 \mathrm{~dB}$ at the resonant frequency

Table 4.3. SAW Resonator Frequency and Attenuation Measurements after Pd Coating

\begin{tabular}{|c|c|c|}
\hline Process & Frequency change & Attenuation change \\
\hline $1000 \AA$ of $\mathrm{SiO}_{2}$ & $0.96 \mathrm{MHz}$ & $-6.8885 \mathrm{~dB}$ \\
\hline $27 \AA$ of Palladium & $0.4 \mathrm{MHz}$ & $-1.64 \mathrm{~dB}$ \\
\hline
\end{tabular}

It was seen that the evaporation of $\mathrm{Pd}$ caused an attenuation of the signal and a corresponding decrease in the resonant frequency of the device. This resonator was then fixed on a PCB and placed in the Stainless Steel cell to be in contact with the incoming gas. This resonator was also made a part of oscillator loop to track the fractional changes in frequency.

The resonator with the pure Pd film was exposed to $0.2-2.5 \%$ volume $\%$ hydrogen in nitrogen at a flow rate of $1000 \mathrm{sccm}$. The response of the sensing film has been plotted 
in Figure 4.17. In accordance with a mass-loading response mechanism, frequency decreases were observed upon hydrogen exposures [11]. Figure 4.18 shows the response times for pure Pd sensing layer, defined as time taken to reach nearly $99 \%$ of complete, for $0.2-2.5 \%$ of hydrogen exposures. It is seen from the plots that the process of absorption and desorption of hydrogen from the Palladium film is reversible, though recovery was slow. Figure 4.19 shows corresponding calibration curve. The calibration curve also shows that the responses obtained for pure palladium sensing film are about $1 / 3$ of Pd coated SWNTs sensing film. Also the pure Pd sensing film shows poor responses at lower concentrations $(<1 \%)$.
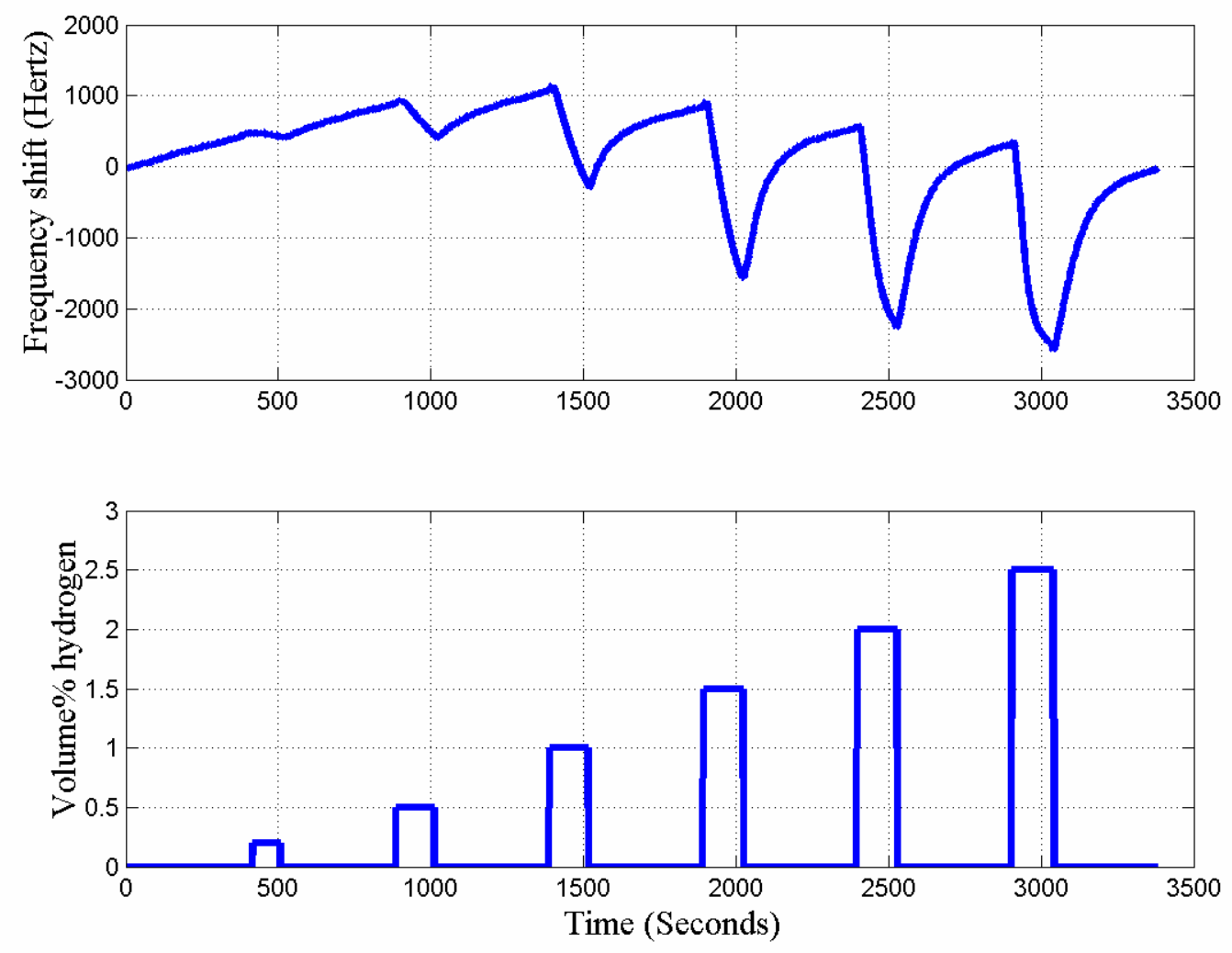

Figure 4.17. The Curve Showing the Response of Pd Film on Exposure to 0.2-2.5\% Hydrogen 


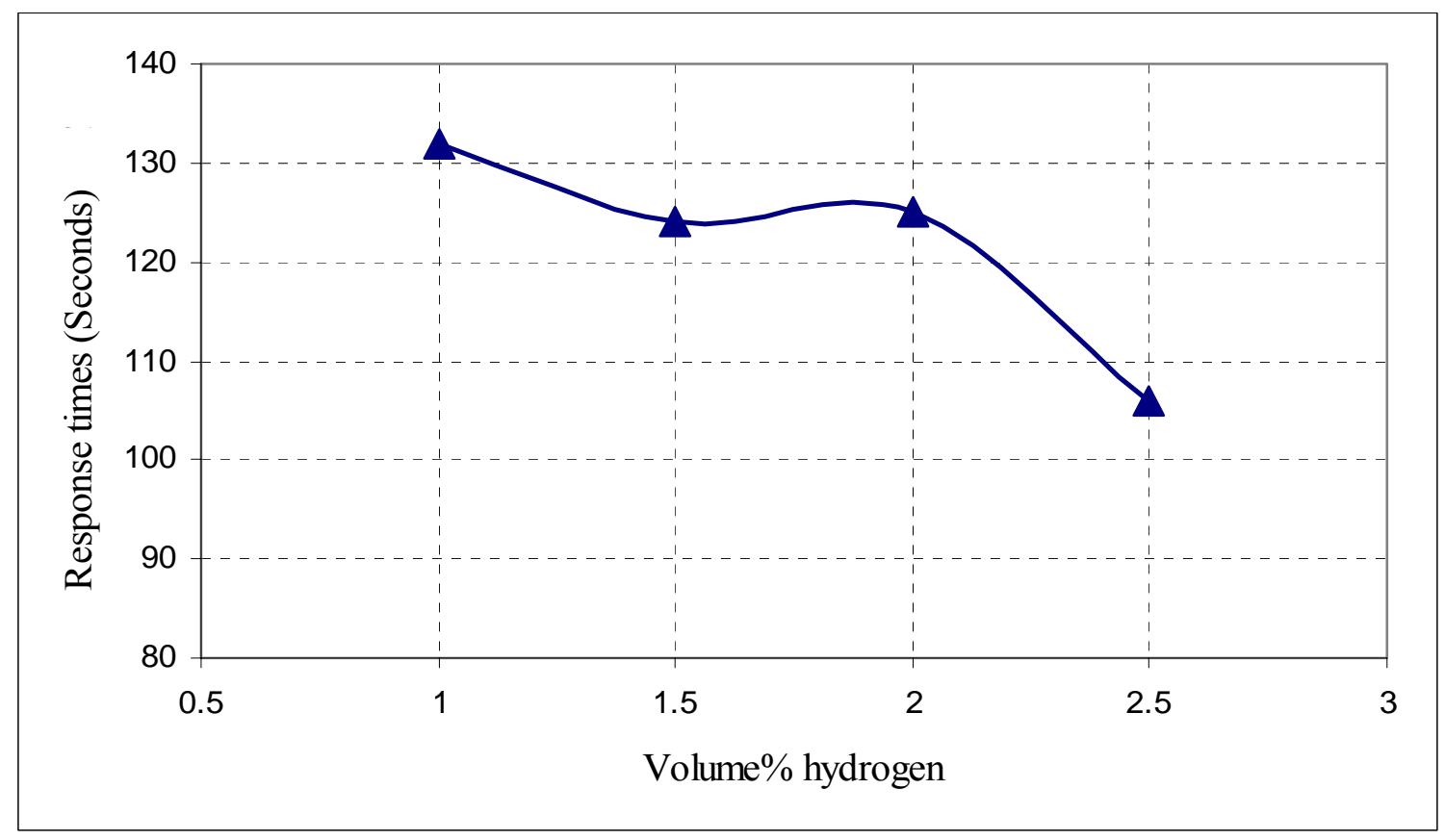

Figure 4.18. Frequency Shifts for Pure Pd Coated SAW Resonator to 1-2.5\% Hydrogen Exposures

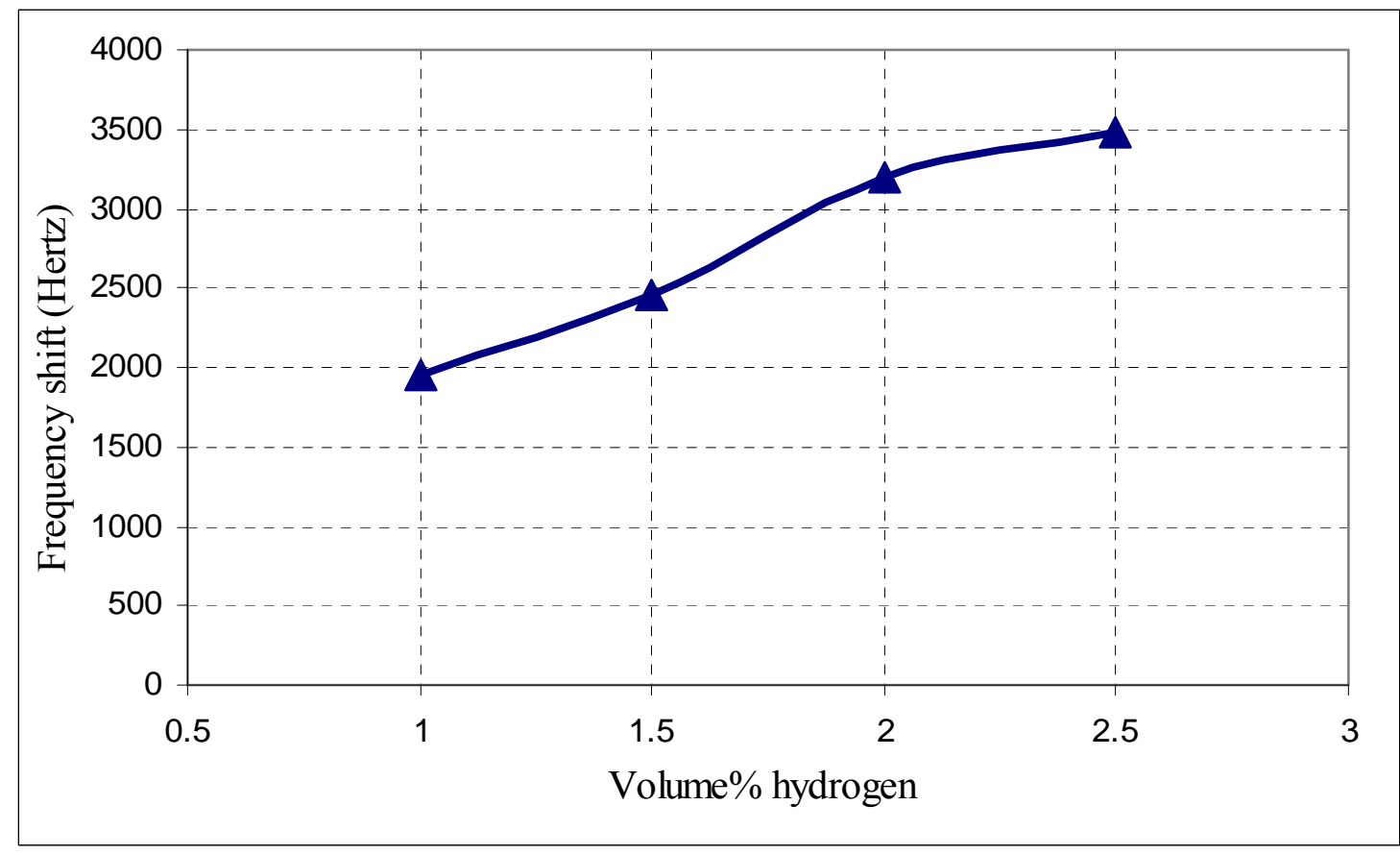

Figure 4. 19. Response Times for Pure Pd Coated SAW Resonator to 1-2.5\% Hydrogen Exposures 


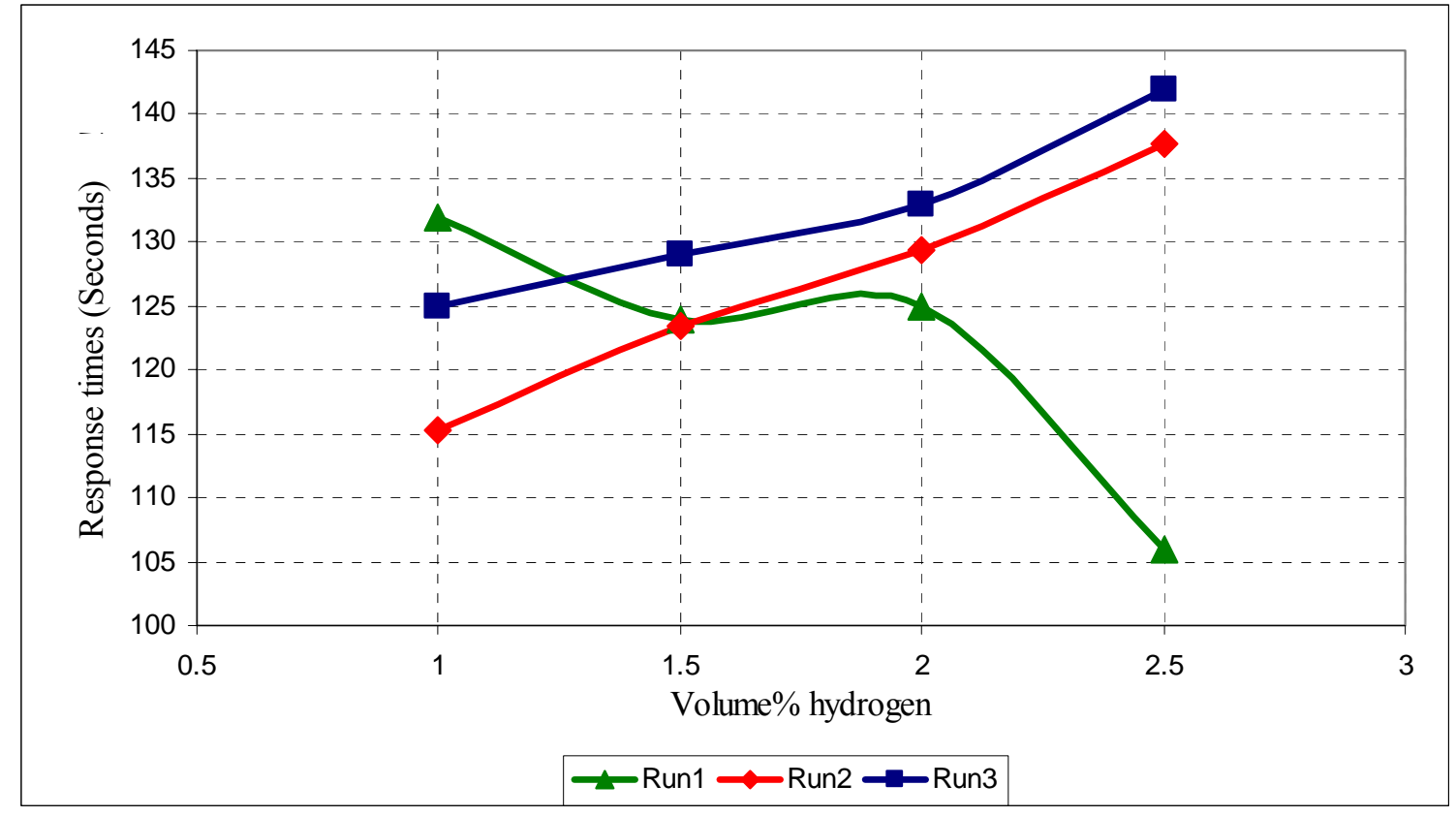

Figure 4.20. Response Times for three different runs of Pure Pd Coated SAW Resonator to $1-2.5 \%$ Hydrogen Exposures

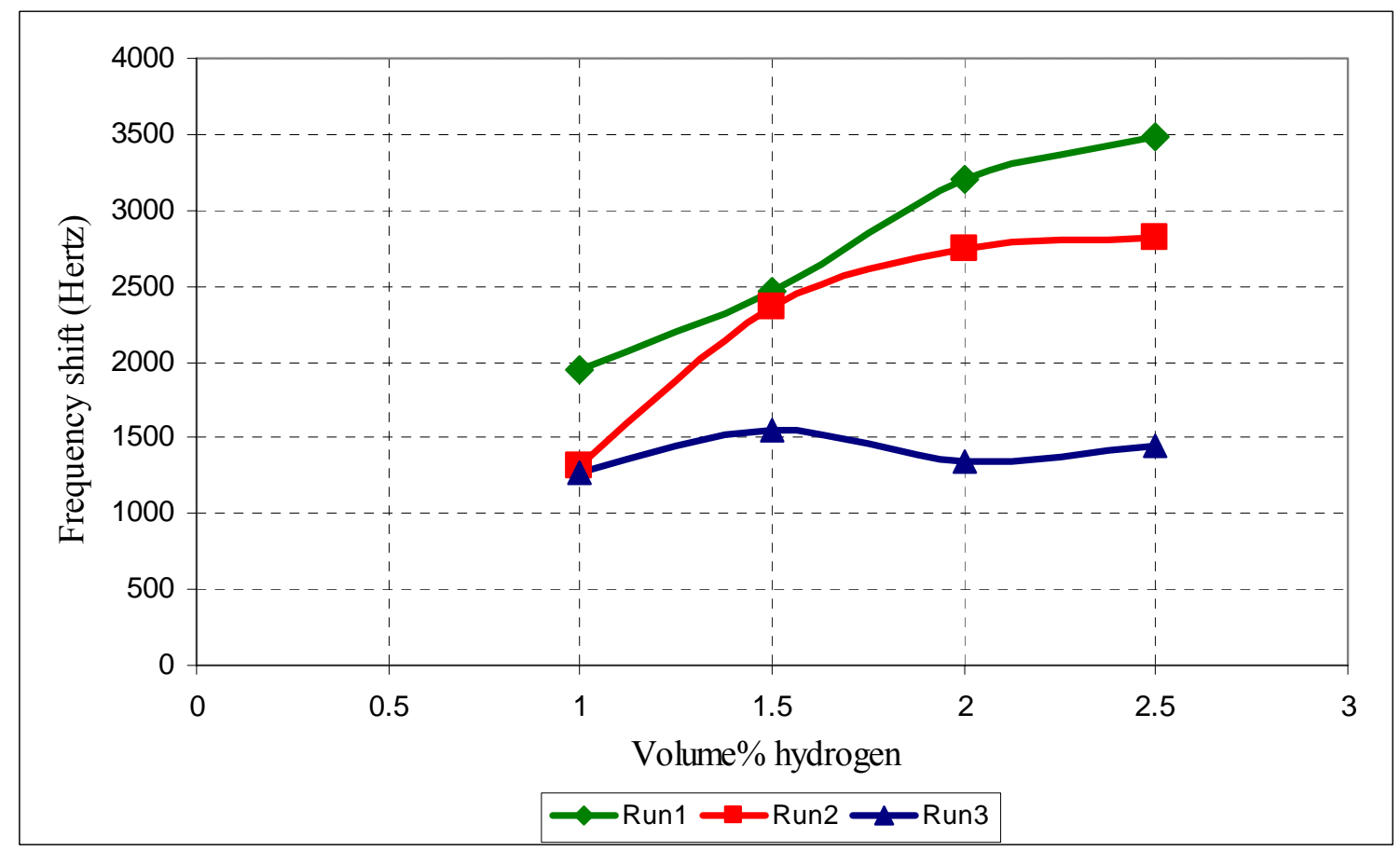

Figure 4.21. Frequency Shifts for three different runs of Pure Pd Coated SAW Resonator to 1-2.5\% Hydrogen Exposures 
A few more runs were done by exposing the Pd coated sensing film to hydrogen to understand the behavior of the film to repeated hydrogen exposures. It can be seen from the plot 4.20 and 4.21 that though the response times are same, the size of shifts is going small.

This shows the degradation of film by repeated exposures, some of which were not seen in Pd coated SWNTs or Pd coated TMV sensing films. The degradation of film can be attributed to change in density of Palladium film at high concentration [36, 37, and 38]. Given the area is fixed the film does not have enough area to expand causing it to crack and peel. This type of behavior was not seen in nanostructures. It could be justified as nanomaterials are dispersed in such a way that there is enough room for expansion, which is the reason why they don't peel of.

\subsection{Comparison of Films}

The three different sensing films were compared for their response times. The response times were compared for time taken to reach $63 \%$ of full scale shift.

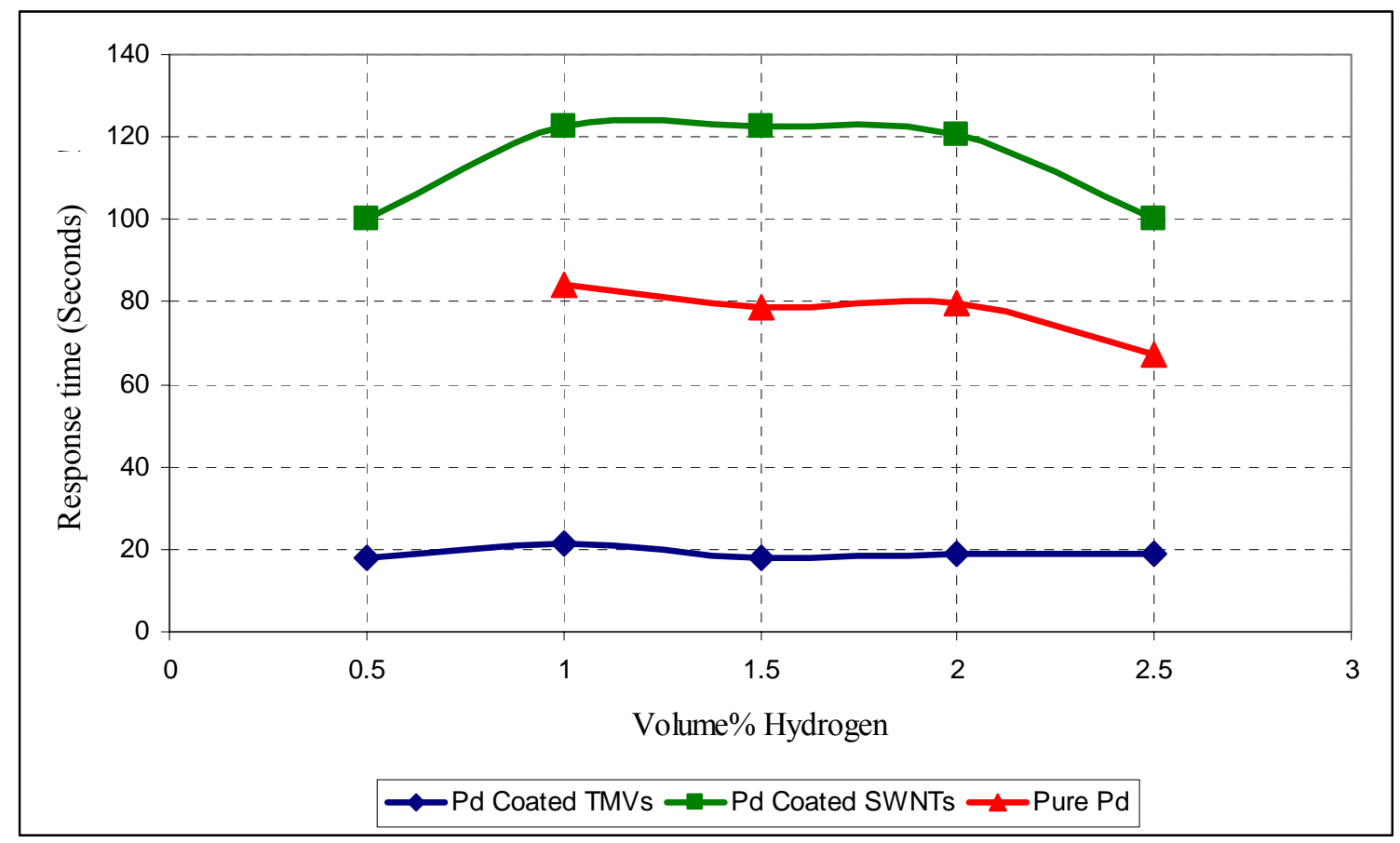

Figure 4.22. The Response Times Observed for the Sensing Films for 63\% of Full

Response 
It was observed that the lowest response times were seen to be for Pd coated TMV virus and highest for Pd coated SWNTs as shown in figure 4.22. Also the amount of frequency shifts produced for $63 \%$ of full scale shift is also plotted in figure 4.23 . It is sent that the highest frequency shifts were observed for Pd coated SWNTs while lowest for Pd coated TMV sensing film. The table 4.4 compares the various sensor parameters for the three films.

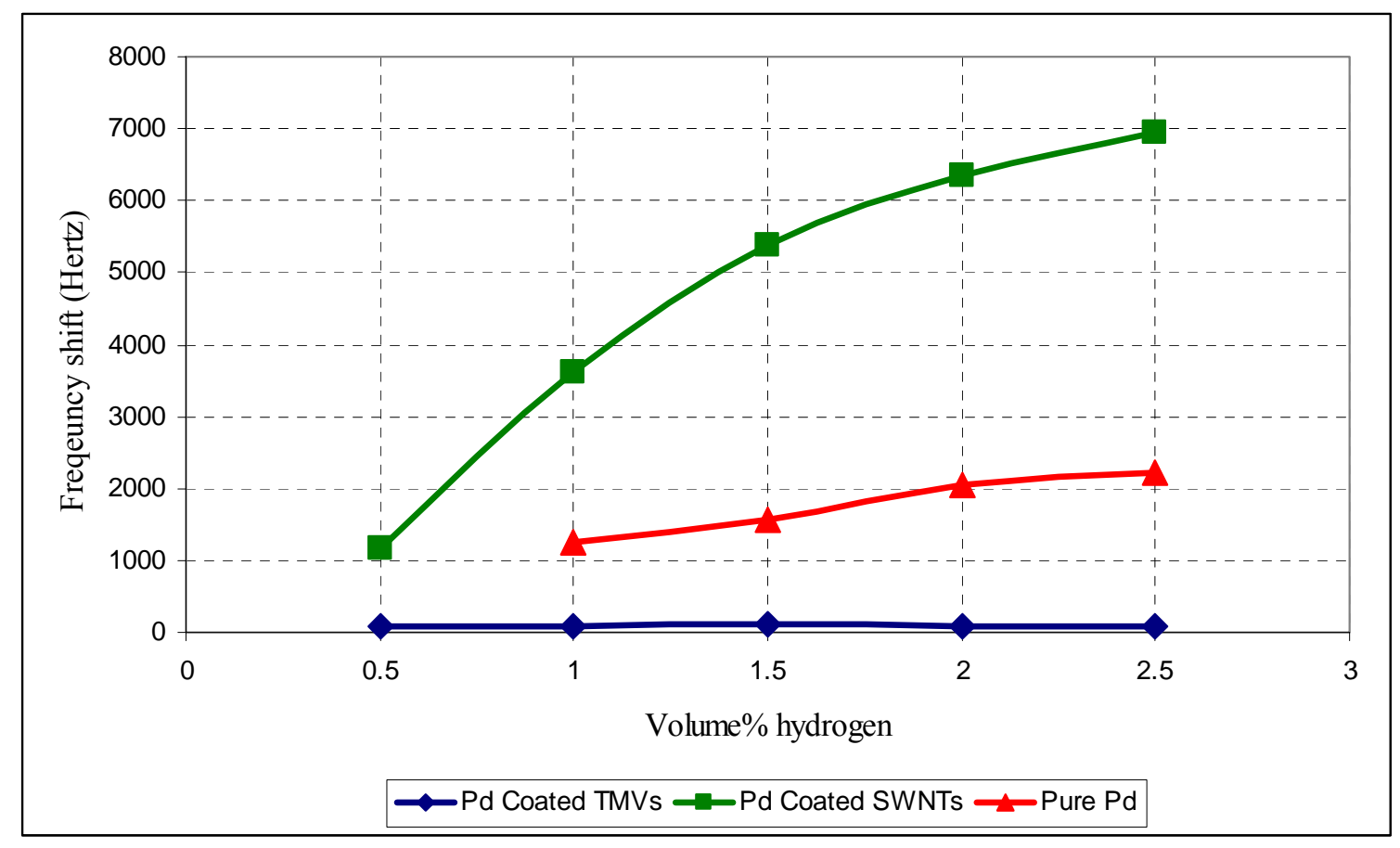

Figure 4.23. The Frequency Shifts Observed for the Sensing Films for 63\% of Full Scale Response

Table 4.4. Comparison of Different Sensor Parameters for the Three Sensing Films

\begin{tabular}{|c|c|c|c|}
\hline & Pd coated TMV & Pd coated SWNTs & Pure Pd \\
\hline Reversible & Yes & Yes & Yes \\
\hline Response Mechanism & Acousto-electric & Mass loading & Mass Loading \\
\hline
\end{tabular}


Table 4.5. Continued

\begin{tabular}{|c|c|c|c|}
\hline $\begin{array}{c}\text { Average Response } \\
\text { time-63\% of full } \\
\text { scale shift (Seconds) }\end{array}$ & 19.09091 & 113.1943 & 77.47727 \\
\hline $\begin{array}{c}\text { Frequency shift- } \\
63 \% \text { of full scale } \\
\text { shift(Hertz) }\end{array}$ & 93.54545 & 3627.91 & 1242.182 \\
\hline $1 \%$ & 115.1818 & 5370.27 & 1568 \\
\hline $1.5 \%$ & 94.18182 & 6342.00 & 2036.364 \\
\hline $2 \%$ & Yes & Yes & No \\
\hline $\begin{array}{c}\text { Repeatability } \\
(50-60 \text { cycles over } \\
8 \text { days })\end{array}$ & Yes(6-8 different & $\begin{array}{c}\text { Yes } \\
\text { tevices were }\end{array}$ & N.A. \\
\hline $\begin{array}{c}\text { Reproducibility } \\
\text { devices were tested) }\end{array}$ & -ive** & -ive** \\
\hline $\begin{array}{c}\text { Direction of shifts } \\
\text { Cycling (requires } \\
\text { exposure for few } \\
\text { cycles before } \\
\text { responding } \\
\text { correctly) }\end{array}$ & + Nov & No & Yes \\
\hline Film robustness & Yes & Yes & No \\
\hline
\end{tabular}

* The resonant frequency increases after analyte absorption by the sensing film

** The resonant frequency decreases after analyte absorption by the sensing film 


\section{CHAPTER 5: CONCLUSION}

In summary we tested three sensing films to see the response to hydrogen challenges. The two nonmaterial sensing films, Pd coated TMV and Pd coated SNWTs were compared with pure Pd film to observe if there is any advantage of using nanomaeterial gas sensing films.

The Pd-TMV film produced small, but easily measurable responses with fast response and recovery times for nearly full response. Frequency increases were observed upon hydrogen exposure, which is unexpected and generally associated with an electroacoustic response mechanism. The Pd-SWNT layers produced much larger shifts, for a very small coating thickness of $27 \AA$ of evaporated Pd on the SWNTs. The frequency decreases observed upon hydrogen exposure are the expected behavior for a mass-loaded SAW sensor response. Shifts were very large and were in the $\mathrm{kHz}$ range. The $99 \%$ response time was somewhat larger at 150 - 200 seconds, than for the Pd-TMV layer. This behavior of nanomaterials was compared to $27 \AA$ Pd film on SAW resonator. The sensing layer responded by producing a decrease in frequency in accordance with mass loading effect for hydrogen challenges and previous literature. These shifts were also seen to be in $\mathrm{KHz}$ range.

The process of absorption and desorption was reversible for all three nanomaterials under room conditions. The SWNT sensing film showed highest frequency shifts for any given concentration, with the smallest shifts observed for Pd coated TMV layer. Both TMV and SWNTs film showed no change in the responses sizes, response times or film behavior due to repeated exposure to hydrogen. The behavior was however not observed in pure Pd film wherein repeated exposure resulted in the size of shifts decreasing with no appreciable changes in response times. This shows a degradation of sensing film with time and use. At high concentrations the Pd film density changes which causes the film to expand. As the film doesn't have enough room for expansion they tend 
to crack and peel. This behavior is not seen in nanostructures as they are dispersed in such a manner that there is enough room for expansion. Both Pd coated TMV and Pd coated SWNTs showed good repeatilibty when tested for over 50 cycles over a span of 8 days which was not seen with pure Pd films. Also the TMV and SWNT sensing film showed reproducibility.

For same level of Pd (27 $\AA)$ in pure Pd and SWNT film the amount of shift was seen to be three times higher for SWNT as compared to pure Pd films. At concentrations below 10000ppm the pure Pd film did not give definite responses which may be related to the need of cycling for pure Pd films. The SWNT film had no problems in giving clear and distinguishable response at lower concentrations. But the response times of SWNT sensing film was seem to be higher than Pd film. This can be explained by the behavior of SWNTs. SWNTs are known to store hydrogen which may be causing the response times to increase as it might take longer than usual for the both Pd and the SWNTs to achieve equilibrium. SWNTs are capable of storing hydrogen in the $5-10 \mathrm{wt} \%$ range and if catalytic metal species are present SWNTs can adsorb up to $8 \mathrm{wt} \%$ hydrogen

All these nanomaterials were tested for 2000-25000ppm of hydrogen. Today with commercial hydrogen sensors available from $0-5000 \mathrm{ppm}$, future work would require testing these devices for lower ppm levels (0-2000ppm). These commercial sensors have response times of 15 seconds. Though the TMV sensing material produced $\sim 20 \mathrm{sec}$ of response times comparable to commercial sensors suitable electronics would have to be designed. When compared to commercial hydrogen sensors costing $100 \$$, the sensors designed using the concept discussed in this thesis could be manufactured at a fraction of the cost. Considering the SAW resonator itself costs only 50cents, the entire associated electronics could be designed for under $5 \$$ if large scale manufacturing is adopted.

Also, in future, in order to achieve bigger responses at lower concentration we would need to increase the coating on these devices. This would require us to evaluate the coating thickness experimentally to see if higher coating could generate a higher shift without comprising a lot on response time. Also the nanomaterials sensing film need to be characterized better, particularly the TMV samples. The TMV samples which produce 
an increase in frequency more in accordance with acousto-electric phenomenon can be better evaluated by coating them on SAW delay line configuration or Lithium Niobate wafer which produces a good response to acouscto-electric phenomenon. Temperature effects on the nanomaterials also need to be studied if commercialization is to be achieved as it should be able to work in a wide range of temperature with no appreciable change in response characteristics. The surface morphology before and after exposure need to be evaluated using TEM and AFM to understand the structural changes undergone by the nanomaterials. TEM and AFM measurements on the nanomaterial films will also reveal the mechanisms by which these films are more stable to repeated hydrogen exposures. TMV and SWNT nanomaetrials coated with different metals could be studied to understand their responses with different gases. 


\section{REFERENCES}

1. D. E. Gobina, "Market Research Report: Gas Sensors and Gas Metering Applications and Markets," pp. 0-318, 2005.

2. C. G. Padro, "US department of Energy's Hydrogen program," National Renewable Energy Lab.

3. "National Hydrogen Energy Roadmap: Production, delivery, storage, conversion, applications, public education and outreach," United States Department of Energy, Washington, DC April 2-3 2002.

4. C.Christofedes and A. Mandelis, "Solid State Sensors for trace hydrogen gas Detection," Journal of Applied Physics, vol. 68, pp. R1-R30, 1990.

5. A. Katsuki and K. Fukui, "H2 Selective gas sensor based on SnO2," Sensors and Actuators, vol. B, pp. 30-37, 1998.

6. M. Thompson and D. C. Stone, Surface launched Surface Acoustic Wave sensors Chemical Sensing and Thin film characterization, vol. 144: Wiley Interscience, 1997.

7. H. Wohlten and R. Dessy, Analytical Chemistry, pp. 1458, 1979.

8. H. Wohlten and R. Dessy, Analytical Chemistry, pp. 1458, 1979.

9. H. Wohlten, Sensors and Actuators, vol. 5, pp. 307, 1984.

10. C. K. Campbell, Surface Acoustic Wave Devices for Mobile and Wireless Communication: Academic Press, 1998.

11. Jakubik, W. P., M. W.Urbanczyk, et al. (2002). "Bilayer Structure of Hydrogen detection in a surface acoustic wave sensor system." Sensors and Actuators B(82): 265-271.

12. Jakubik, W. P., M. W. Urnanczyk, et al. (2003). "Palladium and Phthalocyanine bilayer films for hydrogen detection in a surface acoustic wave sensor system." Sensors and Actuators B 96(1-2): 321-328.

13. Penza, M., E.Milella, et al. (1998). "Monitoring of NH3 gas by LB polypyrrolebased SAW sensor." Sensors and Actuators B(47): 218-224. 
14. Penza, M., F. Antolini, et al. (2004). "Carbon Nanotubes as SAW chemical sensors." Sensors and Actuators B(100): 47-59.

15. Penza, M., G. Cassano, et al. (2002). "Identification and quantification of individual volatile organic compounds in a binary mixture by SAW multisensor array and pattern recognition." Measurement Science and Technology 13: 846858.

16. J. D.S. Ballantine, S. J. Martin, A.J.Ricco, G.C.Frye, and R.M.White, Acoustic Wave Sensors. Theory, Design and Physico-Chemical Applications: Academic Press, 1997.

17. M.J.Jaycock and G.D.Parfitt, Chemistry of Interfaces. Chichester, UK: Ellis Horwood, 1981.

18. J.W.Grate and R.A.McGill, Analytical Chemistry, pp. 2162, 1995.

19. J.W.Grate, S.W.Wenzel, and R.M.White, Analytical Chemistry, vol. 63, pp. 1552, 1991.

20. A.W.Snow, W.R.Barger, M.Klusty, H.Wohltjen, and N.L.Jarvis, Langmuir, vol. 2, pp. 513, 1986.

21. D.L.Bartley and D.D.Dominguez, Analytical Chemistry, vol. 62, pp. 1649, 1990.

22. SES. Research, "Single Walled Nanotubes." from http://www.sesres.com/nanotubes.asp. (September, 2005)

23. "TEM image of SWNTs produced by the CoMoCat method." from http://members.cox.net/hongweizhu/research.htm\#2. (September, 2005)

24. "Examples for armchair, zigzag and chiral nanotube a) $(5,5)-$, b) $(9,0)$ - and c) (10, 5)-nanotube." from www.ipc.uni-karlsruhe.de/ mik/seite_195.html. (September, 2005)

25. "High resolution TEM image of a MWNTs." from http://133.5.181.45/ago/images/tem_hr_mwnt.jpg. (September, 2005)

26. F. L. Pfleger and R. J. Zeyen, "Tomato-Tobacco Mosaic Virus Disease."

27. A.A. Balandin and V. A. Fonoberov, "Vibrational Modes of Nano-Template Viruses," Journal of Biomedical Nanotechnology, vol. 1, pp. 90-95, 2005.

28. The American Phytopathological Society, 2001 from http://www.apsnet.org/education/feature/tmv/images/tmvdraw.htm. (September, 2005) 
29. S. Y. Lee, E. Royston, J. Culver, and M. T. Harris, "Improved metal cluster deposition on a genetically engineered tobacco mosaic virus template," Nanotechnology, pp. S435-S441, 2005.

30. A.Chaudhari, "Development of Surface Acoustic Wave Sensors Using Nanostructured Palladium for Hydrogen Detection," in Department of Chemical Engineering, vol. Masters. Tampa: University of South Florida, 2004.

31. J. Freudenberg, Von Schickfus, M., Hunklinger, S., "A SAW immunosensor for operation in liquid using a $\mathrm{SiO}_{2}$ protective layer," Sensors and Actuators, vol. B, pp. 147-151., 2001.

32. F. Josse, Bender, F., Cernosek, R.W., "Guided shear horizontal surface acoustic wave sensors for chemical and biochemical detection in liquids," Analytical Chemistry, pp. 5937-5944, 2001.

33. G. Kovacs, Vellekoop, M.J., Haueis, R., Lubking, G.W., Venema, A., "A Love sensor for (bio)chemical sensing in liquids," Sensors and Actuators, vol. A, pp. 38-43, 1994.

34. B. Jakoby, Vellekoop, M.J., "Analysis and optimisation of Love-wave liquid sensors.," Ferroelectr. Frequnecy Control, pp. 1293-1301, 1998.

35. R. C. Hughes, W. K. Schubert, T. E. Zipperian, and T. A. Plut, "Thin film palladium and silver alloys and layers for metal-insulator semiconductor sensors.," Journal of Applied Physics, vol. 62, pp. 1074-1081, 1987.

36. A.Fabre, E. Finot, J. Demoment, S. Contreras, In situ measurement of elastic properties of PdHx, PdDx and PdTx, J. Alloys Compd.356-357 (2003) 372-376.

37. V.I. Anisimkin, I.M. Kotelyanskii, V.I. Fedosov, C. Caliendo, P.Verardi, E. Verona, Analysis of the different contributions to the response of SAW gas sensors, in: Proceedings of the IEEE Ultrasonics Symposium, 1995, pp. 515-518.

38. K. Yamanaka, S. Ishikawa, N. Nakaso, N. Takeda, T. Mihara, Y. Tsukahara, Ball SAW device for hydrogen gas sensor, in: Proceedings of the IEEE Ultrasonics Symposium, 2003, pp. 299- 302. 


\section{APPENDICES}




\section{APPENDIX A - PRINTED CIRCUIT FABRICATION}

Procedure

1. Make the layout for obtaining the pattern in AutoCAD or any other suitable designer

2. Obtain (print) the layout on a transparency.

NOTE: The PCB has positive photo resist, so exposed areas are removed while developing.

3. Process to prepare developer:

$\begin{array}{ll}\text { Ingredients: } & \mathrm{NaOH}-(6-9) \mathrm{gms} \\ & \mathrm{DI} \text { water }-1000 \mathrm{ml}=1 \text { litre }\end{array}$

Beaker

Mix the $\mathrm{NaOH}$ and DI water and stir it using magnetic stirrer till all the $\mathrm{NaOH}$ crystals are dissolved.

Note: The higher the amount of $\mathrm{NaOH}$ the faster the developing time.

4. Then take the PCB and with the cutter cut into suitable dimension

5. Then take the PCB and place the transparency pattern on it

6. Place the entire system on the exposing module with the side to be exposed facing the light through the transparency.

7. Close the box and set the number of lamps to 5 and set the timer to 3 .

8. Then after the time elapses open and put the PCB in solution (developer)

9. It takes anywhere from 1-2 minutes for developing which can be clearly seen by the removal of the green photo resist thus exposing the Copper. $\mathrm{NaOH}$ solution which is clear also turns green due to this.

10. Now the areas that where not exposed were will still have photo resist on them.

11. Now place the entire PCB in the solution of ferric chloride which causes the copper in unwanted areas to be removed (the areas exposed). The entire process may take anywhere from 20-30 minutes with ferric chloride at $(30-40)^{\circ} \mathrm{C}$. The end of process is indicated by the board becoming transparent.

12. Then put it in acetone to remove the photo resist. 
13. Then cut the board into required dimension.

14. Drill holes where it's required and then complete the circuit. 